\title{
Weight Estimation of Marine Propulsion and Power Generation Machinery
}

\author{
Arun Kr Dev* and Makaraksha Saha** \\ * Newcastle University in Singapore, Singapore, ** M K Marine Pte Ltd, Singapore
}

\begin{abstract}
During the conceptual and preliminary design stage of a ship, designers need to ensure that the selected principal dimensions and parameters are good enough to deliver a stable ship (statically and dynamically) besides deadweight and speed. To support this, the initial intact stability of the proposed ship is required to be calculated, and in doing so, the lightship weight and its detailed breakdown are necessary to be known. After hull steel weight, machinery weight, mainly, marine propulsion and power generation machinery, play a vital role in the lightship weight estimate of a ship due to its robustness. The correct estimation of respective weights improves the accuracy of calculating a ship's initial stability typically to be designed and built. Hence, it would be advantageous for the designer to convince the shipowner.

A total of 3006 marine propulsion (main marine diesel) engines and 348 power generation (auxiliary marine diesel) engines/generators of various power output (generators output for auxiliary engines), engine RPM and cylinder number of different engine makers are collected. These are analyzed and presented in both tabular and graphical forms to demonstrate the possible relationship between marine propulsion engine weight and power generation engine weight, and their respective power output, RPM, cylinder number, power-RPM ratio and power-RPM ratio per cylinder.

In this article, the authors have attempted to investigate the behaviour of marine propulsion engine weight and power generation engine/generator weight regarding engine power output, generator power output, engine RPM and cylinder number (independent variables). Further attempts have been made to identify those independent variables that influence the weight of the marine propulsion engine and power generation engine/generator (dependent variables), and their interrelationships. A mathematical model has thus been developed and proposed, as a guiding tool, for the designer to estimate the weight of main and auxiliary engines more accurately during the conceptual and preliminary design stage.
\end{abstract}

Keywords: engine weight; engine power; engine/generator power; engine RPM; cylinder number; power-RPM ratio; power-RPM ratio per cylinder; low-speed; medium-speed; high-speed; standard deviation; correlation coefficient; coefficient of multiple determination; F-statistic; 


\subsection{Introduction}

The conceptual and preliminary design stage is the beginning of a ship design process, before engaging in a shipbuilding contract between the shipowner and the shipyard. During this stage, designers select all necessary dimensions and parameters to fulfil the ship owner's requirements, the classification society, statutory regulations, etc. Sometimes, different issues, such as deadweight and speed, are required to be finalized during this design stage. Calculation of preliminary intact stability is a part of this process to ensure that the proposed dimensions and parameters are sufficient enough to deliver a stable ship (statically and dynamically). For this stability calculation, the weights of hull, machinery, and outfit, including their positions of the centre of gravity, are needed. Machinery weights are from marine propulsion (main marine diesel) engines (from now on the main engine), power generation engines (from now on the auxiliary engine), shaft generators, various deck machinery, main compressors, different pumps with motors, ballast water treatment plant, sewage treatment plant, hydraulic power unit, steering gear, etc. In short, every item onboard a ship is to be included in the calculation. In this article, only the weights of the main engine and auxiliary engine are included.

There are various ways to calculate the hull steel weight with an acceptable level of accuracy. Still, for various machinery/equipment mentioned earlier, there are no reliable and established formulas or procedures to calculate the appropriate weight. Typically, weights of various machinery/equipment are taken from a similar ship or a particular manufacturer's catalogue. Sometimes, during the machinery/equipment selection process, manufacturers and models are different from the initial selection, and hence the weight. This change in weight (particularly for heavy equipment, such as the main engine, auxiliary engine, deck machinery, etc.) significantly impacts the lightship weight, centre of gravity ( $\operatorname{cog})$, and stability. On the other hand, if the weight is calculated with the help of a reliable and established formula using machinery/equipment parameters, such as power output, RPM and number of cylinder, which are free from the influence of manufacturer and model, the issue of variation in weight can easily be avoided, if not entirely, at least partly.

Data analysis of the main engine's weight (from now on ME weight) is carried out against different independent variables. These are mainly main engine power output at 100\% MCR (hereinafter power output), main engine RPM (hereinafter RPM), number of cylinders of the main engine (hereinafter cylinder number), power-RPM ratio output of the main engine (hereinafter power-RPM ratio) and power-RPM ratio output per cylinder of main engine (hereinafter power-RPM ratio per cylinder). Power output, RPM, cylinder number, power-RPM ratio, and power-RPM ratio per cylinder (independent variables) significantly influence main and auxiliary engines' weight. However, the magnitudes of impact on ME weight vary from variable to variable. 
Similarly, data analysis of auxiliary engine weight (from now on AE weight) is carried out against different independent variables. These are mainly auxiliary engine electrical power output at $100 \%$ MCR (hereinafter power output), auxiliary engine RPM (hereinafter RPM), number of cylinders of the auxiliary engine (hereinafter cylinder number), power-RPM ratio output (hereinafter power-RPM ratio), power-RPM ratio output per cylinder (hereinafter power-RPM ratio per cylinder). Power output, RPM, cylinder number, power-RPM ratio, and power-RPM ratio per cylinder (independent variables) significantly impact the AE weight. However, the magnitudes of impact on weight vary from variable to variable.

Diesel engines may be classed as V-type and inline (I)-type in a broader spectrum. There are inherent differences between V-type and I-type engines concerning geometrical configurations and design considerations. Design aspects of marine diesel engines are beyond this article's scope and hence, are not covered. However, these physical differences affect the weight of engines. This article covers only I-type engines, not V-type engines, to obtain a more stable, reliable, and realistic relationship among the variables, as mentioned earlier.

This article aims to investigate and establish the interrelationship among the dependent variable and the independent variables responsible for machinery weight. Data collection and analysis (both analytical and graphical) are carried out to meet the aim.

The remainder of the article is arranged as follows: a review of the related literature is presented in Section 2. In Section 3, problem formulation and associated assumptions are discussed. Collected samples, initial findings, and methodology are presented in Section 4. Analysis of weight (main engine and auxiliary engine) is shown in graphical form in Section 5. In Section 6, the results of the investigation and application of findings are presented. Finally, Section 7 concludes the article and proposes suggestions for future research work.

\subsection{Literature review}

Very few studies were conducted about the engine weight for their parameters, such as power output, RPM, and cylinder number. Watson (1962) initiated the very first study of this kind. The author has expressed the engine weight as a function of the power-RPM ratio $\left(\frac{M C R}{R P M}\right)$ in the form of a simple linear equation, $y=\mathrm{m} * x+\mathrm{c}$. Also, in this study, diesel engines and turbine machinery were taken into consideration to formulate the equation for estimation of engine weight. Surprisingly, the maximum power shown was only 15,000 SHP, on that date, installed on a limited number of passenger ships. The then 'high-speed diesel' (today's medium-speed engines) tailed off at 3,500 SHP.

Watson and Gilfillan (1976) re-visited the earlier article (Watson 1962). The authors proposed the engine weight as a function of the power-RPM ratio $\left(\frac{\mathrm{MCR}}{\mathrm{RPM}}\right)$, but in the form of a power equation, $y=$ $\mathrm{m} * x^{\mathrm{n}}$. Also, in the article, various types of engines, such as direct-drive and geared drive, diesel and 
steam, turbo-electric and diesel-electric, nuclear power, and so on, were considered. It is highlighted that the proposed power equation yields an estimated engine weight $5 \%$ higher than presented the line through the data spots to allow for the fact that the graph ought to be a stepped line corresponding to cylinder numbers with approximately $10 \%$ weight steps for the addition of each cylinder. The article also suggested a procedure about the estimation of remaining components of machinery weight.

Schneekluth and Bertram (1998) proposed 'weight - power" linear relationship of a diesel engine under engine speed groups like slow speed (110 - $140 \mathrm{rpm}$ ), medium speed (400 - $500 \mathrm{rpm}$ ) and medium speed V-type engines ( $400-500 \mathrm{rpm}$ ). Authors claimed that the proposed relationship would produce a reasonable estimate of engine weight (dry engine - without cooling water and lubricant). Roh and Lee (2018) studied the machinery weight from different angles. They considered the machinery (main engine) weight as a lightweight component together with a ship's structural weight and outfit weight to estimate the lightweight. Authors assumed that the main engine's weight is directly proportional to NMCR (Nominal Maximum Continuous Rating) of the engine. Using the admiralty equation, they finally proposed a non-linear equation to estimate the main engine weight involving length, breadth, draught, block coefficient and service speed.

Papanikolaou (2014) mentioned the importance of the main machinery (main engine) and gearbox as part of the machinery weight. The importance of main machinery weight also took the effects of slowspeed, medium-speed and high-speed. While many approximate formulas and other approximations have been provided for the total machinery weight, very little has been mentioned about calculating the main machinery weight in the design stage.

\subsection{Problem formulation and related assumptions}

\subsection{Problem formulation}

After hull (steel) weight, different machinery weight, mainly, main engines (ME) and auxiliary engines (AE) contribute the most to the lightship weight and centre of gravity (cog). The latter has the maximum impact on the initial intact stability, which is usually calculated at the conceptual and preliminary design stage of a ship. During this stage, in most cases, leading equipment makers and their models are not known. Designers assume a model from a particular maker which, later on, may not be the final one. Hence, the weight and cog calculations do not reflect the ship's performance on stability. As such, designers need to re-calculate the stability using the data of finally selected maker and model.

This situation can be improved dramatically if a reliable guideline about the relationship between engine weight and other engine parameters is available. Keeping this problem in mind, this research focuses on the interrelationship among engine weight and various parameters of engines, such as 
power output, RPM, and cylinder number. Based on the relationship, an engine's weight can be estimated to solve the problem mentioned earlier, at least partly, if not entirely.

\subsection{Related assumptions}

\subsubsection{Engine power output}

The engine power output refers to the engine's power at $100 \%$ maximum continuous rating (MCR) condition. The higher power output requires a bigger/longer engine dimensionally, and a bigger/longer engine means more weight irrespective of RPM and cylinder number. The power output can also be increased by adding additional cylinders, which results in extra weight. Therefore, the power output has a positive impact on the weight irrespective of RPM and cylinder number, and this is expected to be linearly associated. Hence, the first assumption is, ceteris paribus, the weight is a function of power output and assumed to be linearly associated.

\subsubsection{Generator power output}

The generator output refers to the electrical power delivered by a generator. In this research, the generator is driven by a diesel engine. The power output of the diesel engine is higher than that of a generator. Typically, diesel engine power output is about 4-5\% higher than that of a generator output (Fig.1). A higher generator output requires a higher engine power output and a more significant size generator, resulting in the generator and engine's higher weight irrespective of RPM and the diesel engine's cylinder number. Therefore, the generator power output has a positive impact on weight. Hence, the second assumption is, ceteris paribus, the weight is a function of the power output and is expected to be linearly associated.

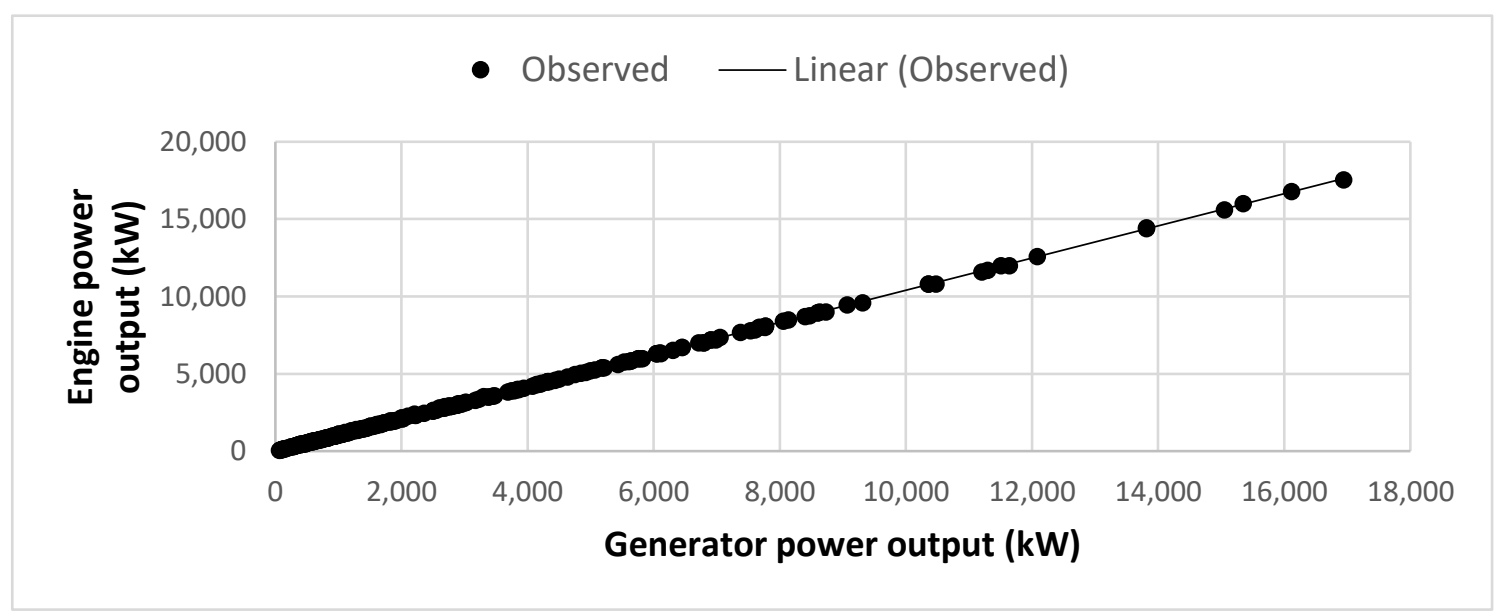

Fig. 1 Power output of engine versus generator of auxiliary engines

\subsubsection{Engine RPM}

The RPM of an engine refers to the number of revolutions of the crankshaft per minute (RPM). Technically, keeping power output and cylinder number same for a particular engine, higher speed means lower power-RPM ratio. It causes smaller dimensions (maybe shorter stroke length, smaller 
cylinder bore diameter, and other scantlings) resulting in lesser weight. Therefore, RPM of an engine has a negative impact on the weight irrespective of power output and cylinder number and is expected to be linearly related. Hence, the third assumption is, ceteris paribus, weight is a function of RPM (negatively) and assumed to be linearly associated.

\subsubsection{Cylinder number}

Without any elaboration, it can be said that the cylinder number of an engine has a direct impact on the power output and hence weight. Therefore, cylinder number has a positive effect on weight irrespective of power output and RPM and is expected to be linearly associated. Accordingly, the fourth assumption is, ceteris paribus, weight is a function of cylinder number and assumed to be linearly associated.

\subsubsection{Engine power-RPM ratio output}

The power-RPM ratio output of an engine is defined by the ratio of power output to RPM, $\left(\frac{\text { Power }}{\text { RPM }}\right)$, at $100 \%$ MCR. Technically, an increase in power-RPM ratio means a reduction in RPM at constant power output. A decrease in RPM implies an increase in weight. Therefore, the weight has a positive impact on power-RPM ratio. Hence, the fifth assumption is, ceteris paribus, the weight is a power-RPM ratio function and is expected to be linearly associated.

Mathematically, the power output of an engine may be expressed in different units, like, HP (Horsepower), kW (kiloWatt), and PS (Metric horsepower). Torque may also be expressed in different units, like, lb-ft (Pound feet), N-M (Newton meter) and kg-M (Kilogram meter). An appropriate conversion factor is required for each combination of power to torque conversion and vice versa. To avoid this hassle, instead of torque, the ratio of power to RPM $\left(\frac{\text { Power }}{\operatorname{RPM}}\right)$ is used throughout this article. This is also convenient to use in a practical field to compare and validate the findings with other available results, which also used the same ratio.

\subsubsection{Engine power-RPM ratio output per cylinder}

Like the power-RPM ratio, the power-RPM ratio per cylinder $\left(\frac{P \text { ower }}{\operatorname{RPM} * N}\right)$ has a positive impact on engine weight. Hence, the sixth assumption is, ceteris paribus, the weight is a function of power-RPM ratio per cylinder and is expected to be linearly associated.

So far, it has been highlighted theoretically that engine power output, RPM, cylinder number, powerRPM ratio, and power-RPM ratio per cylinder are linearly associated with the corresponding weight of an engine. In other words, engine weight is a linear function of power output, RPM, cylinder number, power-RPM ratio, and power-RPM ratio per cylinder. Mathematically, the above assumptions can be expressed in equations (1-5) as follows.

$W_{\text {engine }}=f($ Power output $)=\mathrm{a}_{1}+\mathrm{b}_{1} * P$ 
$W_{\text {engine }}=f($ Cylinder number $)=\mathrm{a}_{2}+\mathrm{b}_{2} * N$

$W_{\text {engine }}=f(R P M)=\mathrm{a}_{3}+\mathrm{b}_{3} * R P M$

$W_{\text {engine }}=f($ Power $-R P M$ ratio $)=\mathrm{a}_{4}+\mathrm{b}_{5} * T$

$W_{\text {engine }}=f$ (Power $-R P M$ ratio per cylinder $)=\mathrm{a}_{5}+\mathrm{b}_{5} * T / N$

Where $W_{\text {engine }}$ is the weight of the engine

$P$ is the power output $(\mathrm{kW})$ at $100 \%$ MCR for the main engine (ME) and

electrical power output ( $k W$ ) for the auxiliary engine $(A E)$

$N$ is the cylinder number of engine

$R P M$ is speed (revolution per minute)

$T$ is the power-RPM ratio $(P / R P M)$

\subsection{Data collection and methodology}

Studying the behavior/pattern of the weight of main engines and auxiliary engines, the detailed information of main engines, and auxiliary engines must be known. For this purpose, details of main engines and auxiliary engines of various manufacturers with different power output, RPM, cylinder number were collected and compiled.

\subsection{Marine propulsion machinery weight}

To develop the weight function and verify the assumptions, a total of 3,306 marine diesel engines' data of I- and V-type were compiled from different manufacturers' product list. All these engines are in production from time to time.

A general picture of sample engines is presented in Tables 1-2 and Figs.2-8. Table 1 summarizes several engines, average RPM, power output, and weight against the cylinder number for V-and I-type engines. One can easily understand the average RPM, power output, and weights of V- and I-type engines of same cylinder number. It also appears that for main engines of a particular cylinder number, these values are far apart for $\mathrm{V}$ - and I-type.

Table 1 Summary of main engines by V-type and I-type

\begin{tabular}{|c|c|c|c|c|r|r|r|r|r|}
\hline Serial & Cylinder & \multicolumn{3}{|c|}{ Average for V-type engines } & \multicolumn{4}{|c|}{ Average for l-type engines } \\
\cline { 2 - 9 } No. & number & No. & RPM & Power & Weight & No. & RPM & Power & Weight \\
\hline 1 & 4 & & & & & 17 & 141 & 8,551 & 283 \\
\hline 2 & 5 & & & & & 567 & 117 & 7,521 & 241 \\
\hline 3 & 6 & & & & & 787 & 306 & 8,679 & 269 \\
\hline 4 & 7 & & & & & 647 & 134 & 11,971 & 368 \\
\hline 5 & 8 & 15 & 1,507 & 682 & 5 & 685 & 174 & 11034 & 389 \\
\hline 6 & 9 & & & & & 179 & 263 & 21,286 & 666 \\
\hline 7 & 10 & & & & & 40 & 147 & 45,345 & 1,520 \\
\hline 8 & 11 & & & & & 36 & 82 & 54,740 & 1,838 \\
\hline 9 & 12 & 151 & 1,478 & 3,066 & 31 & 38 & 119 & 56,855 & 1,892 \\
\hline
\end{tabular}




\begin{tabular}{|l|l|r|r|r|r|r|r|r|r|}
10 & 13 & & & & & 4 & 97 & 61,360 & 2,160 \\
\hline 11 & 14 & 12 & 599 & 13,159 & 167 & 4 & 97 & 66,080 & 2,300 \\
\hline 13 & 16 & 99 & 1,267 & 5,593 & 58 & 2 & 875 & 6,740 & 59 \\
\hline 15 & 18 & 14 & 861 & 13,543 & 158 & & & & \\
\hline 17 & 20 & 4 & 876 & 10,775 & 78 & & & & \\
\hline
\end{tabular}

Table 2 is constructed using Table 1. It shows a comparison of some selected characteristics, such as weight per unit power output, weight per unit power-RPM ratio output and power output per unit cylinder, of V-type and I-type main engines against cylinder number. Table 2 shows that the weight per unit power output for $\mathrm{V}$-type engines ranges from 7 to 13 and for I-type engines varies from 8 to 35. The weight per unit power-RPM ratio $\left(\frac{M C R}{R P M}\right)$ for V-type engines varies from 6,341 to 14,944 and for I-type engines varies from 2,753 to 9,484 . The power output per unit cylinder for V-type engines varies from 85 to 940 and for I-type engines varies from 421 to 4,976. It is very clear from Table 2 that the magnitudes of the characteristics mentioned above for V-type and I-type engines having the same cylinder number are far apart from each other. This is probably because of inherent differences between V-type and I-type concerning geometrical configurations and design considerations. As such, it is not appropriate to combine both types in the same platform for analysis. Therefore, V-type engines are excluded from the analyses in this investigation. Only I-type engines are considered to ensure consistency, uniformity, and reliability in the analyses' results.

Table 2 Summary of characteristics of V-type and I-type main engines

\begin{tabular}{|c|c|c|c|c|c|c|}
\hline \multirow{2}{*}{$\begin{array}{l}\text { Cylinder } \\
\text { number }\end{array}$} & \multicolumn{2}{|c|}{ Weight/power (kg/kW) } & \multicolumn{2}{|c|}{ Weight/power-RPM ratio $(\mathrm{kg} / \mathrm{T})$} & \multicolumn{2}{|c|}{ Power/cylinder (kW/N) } \\
\hline & V-type & I-type & V-type & I-type & V-type & I-type \\
\hline 4 & & 33.10 & & 4,666 & & 2,138 \\
\hline 5 & & 32.04 & & 3,749 & & 1,504 \\
\hline 6 & & 30.99 & & 9,484 & & 1,447 \\
\hline 7 & & 30.74 & & 4,119 & & 1,710 \\
\hline 8 & 7.33 & 35.25 & 11,048 & 6,134 & 85 & 1,379 \\
\hline 9 & & 31.29 & & 8,229 & & 2,365 \\
\hline 10 & & 33.52 & & 4,928 & & 4,535 \\
\hline 11 & & 33.58 & & 2,753 & & 4,976 \\
\hline 12 & 10.11 & 33.28 & 14,944 & 3,960 & 256 & 4,738 \\
\hline 13 & & 35.20 & & 3,415 & & 4,720 \\
\hline 14 & 12.69 & 34.81 & 7,602 & 3,376 & 940 & 4,720 \\
\hline 16 & 10.37 & 8.75 & 13,139 & 7,659 & 350 & 421 \\
\hline 18 & 11.67 & & 10,045 & & 752 & \\
\hline 20 & 7.24 & & 6,341 & & 539 & \\
\hline
\end{tabular}

Figures 2-8 show the distribution of the main engine type, cylinder number, RPM, power output, power-RPM ratio, the power-RPM ratio per cylinder, and weight. These figures also depict the limiting 
values of the respective parameters. It can be seen from Fig. 2 that $91 \%$ of sample main engines are of the I-type. Figure 3 demonstrates the distribution of cylinder number with an average value of 7 and $67 \%$ of sample main engines are within 7 cylinders range. Figure 4 illustrates RPM's distribution with an average value of 193 RPM and 87\% of sample main engines are within 193 RPM range. Figure 5 demonstrates the distribution of power output with an average value of $12,705 \mathrm{~kW}$ and $71 \%$ of sample main engines are within 12,705 kW power output range. Figure 6 demonstrates the distribution of power-RPM ratio with an average value of 1,347 and $79 \%$ of sample main engines are within the 1,347 power-RPM ratio range. Figure 7 displays the distribution of power-RPM ratio per cylinder with an average value of 184 and 71\% of sample main engines are within 184 power-RPM ratios per cylinder range. Figure 8 demonstrates the weight distribution with an average value of 397 tonnes $\left(\mathrm{kg} \times 10^{3}\right)$ and $71 \%$ of sample main engines are within 397 tonnes $\left(\mathrm{kg} \times 10^{3}\right)$ weight range.

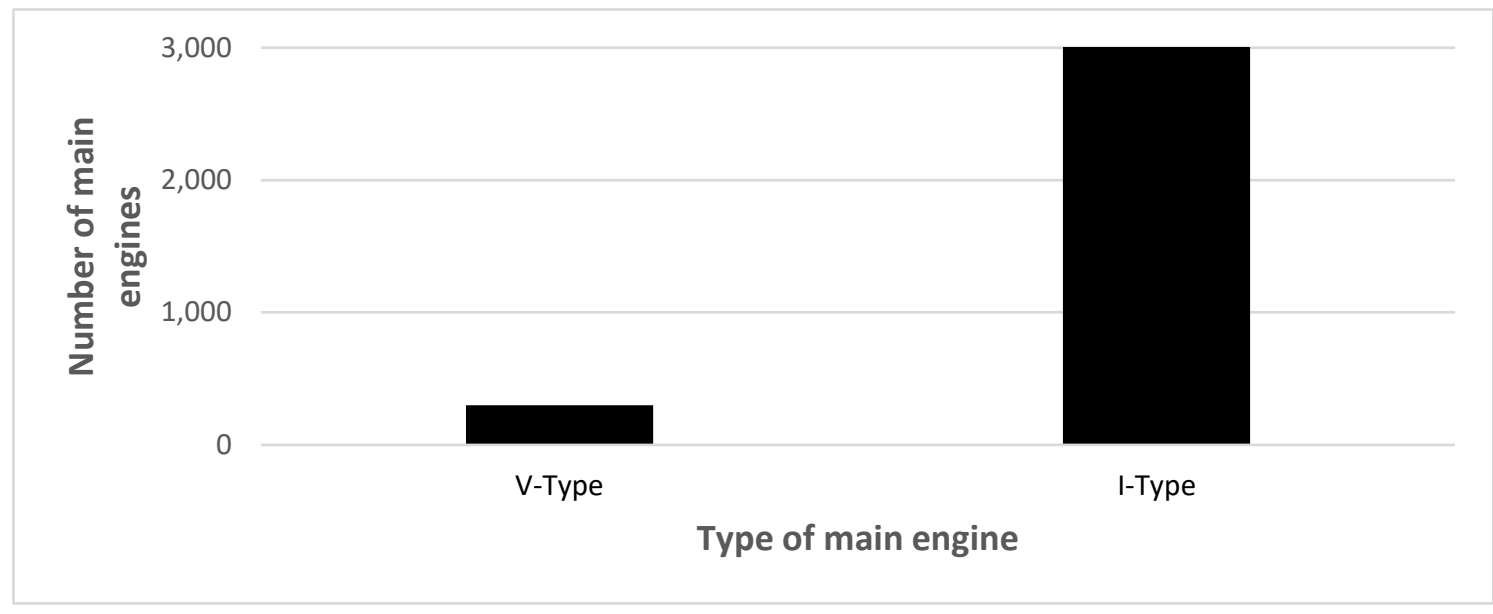

Fig.2 Distribution of type of main engines

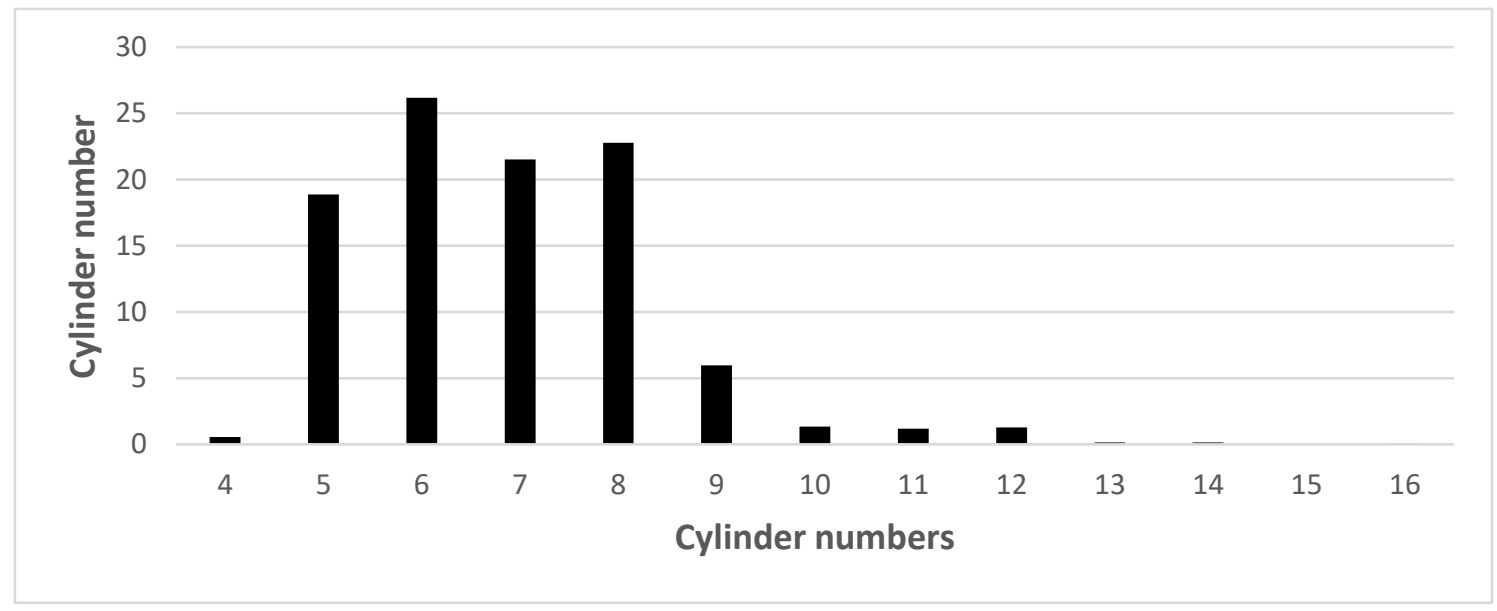

Fig.3 Distribution of cylinder number of I-type main engines 


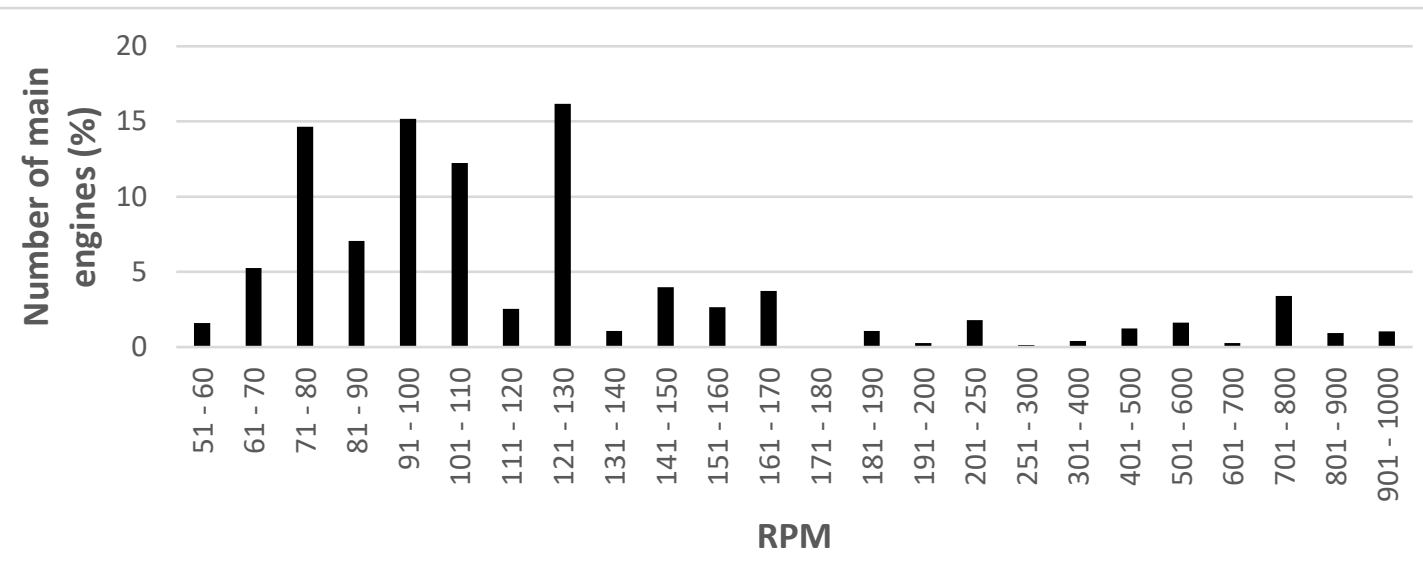

Fig.4 Distribution of RPM of I-type main engines

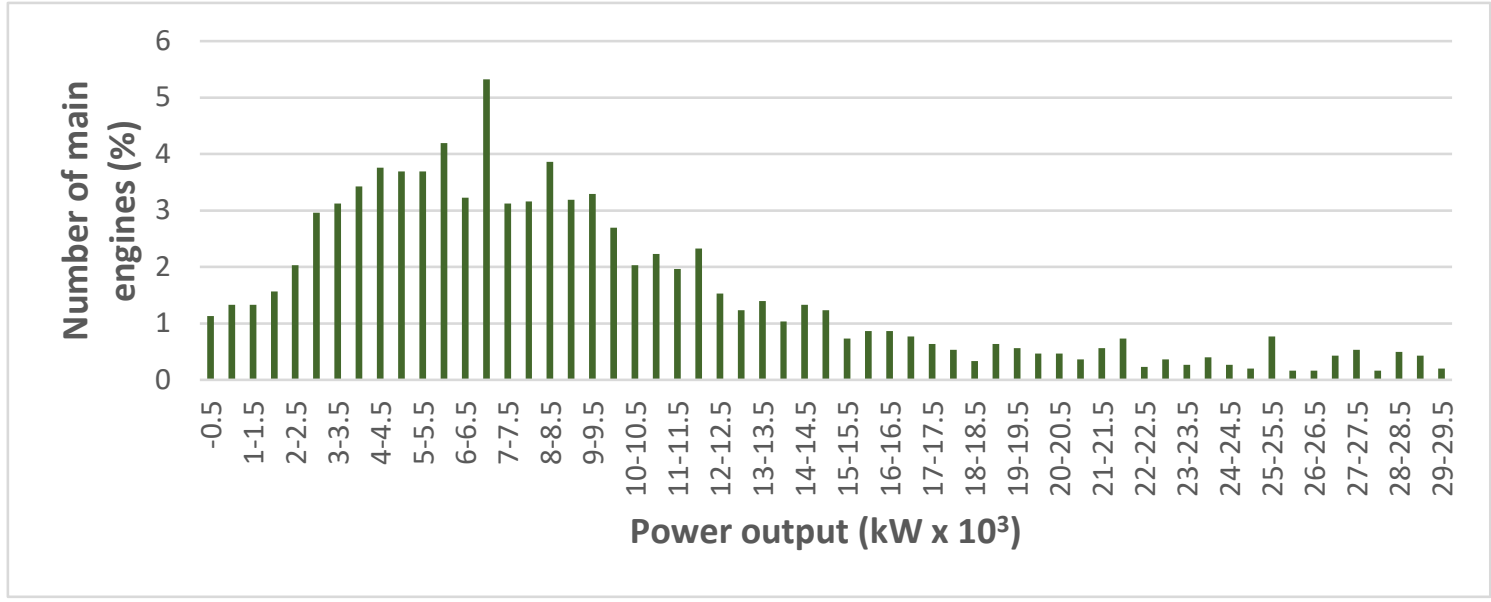

Fig.5 Distribution of power output of I-type main engines

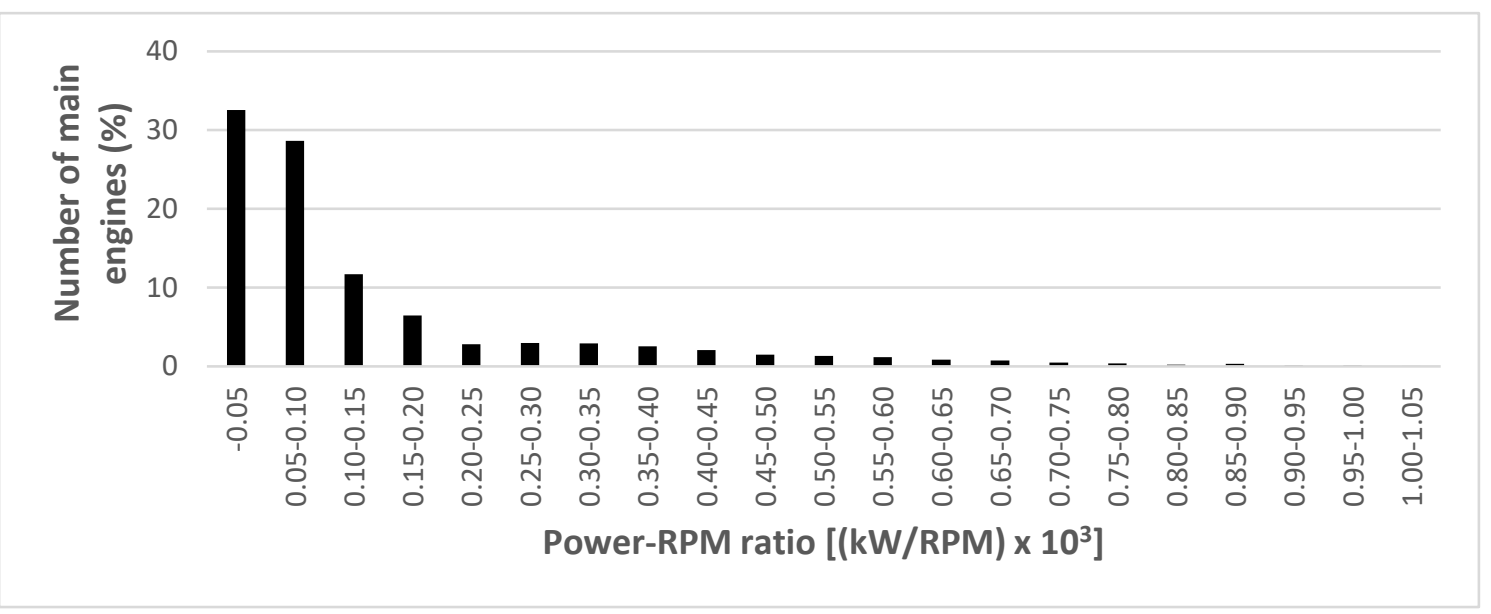

Fig.6 Distribution of power-RPM ratio of I-type main engines 


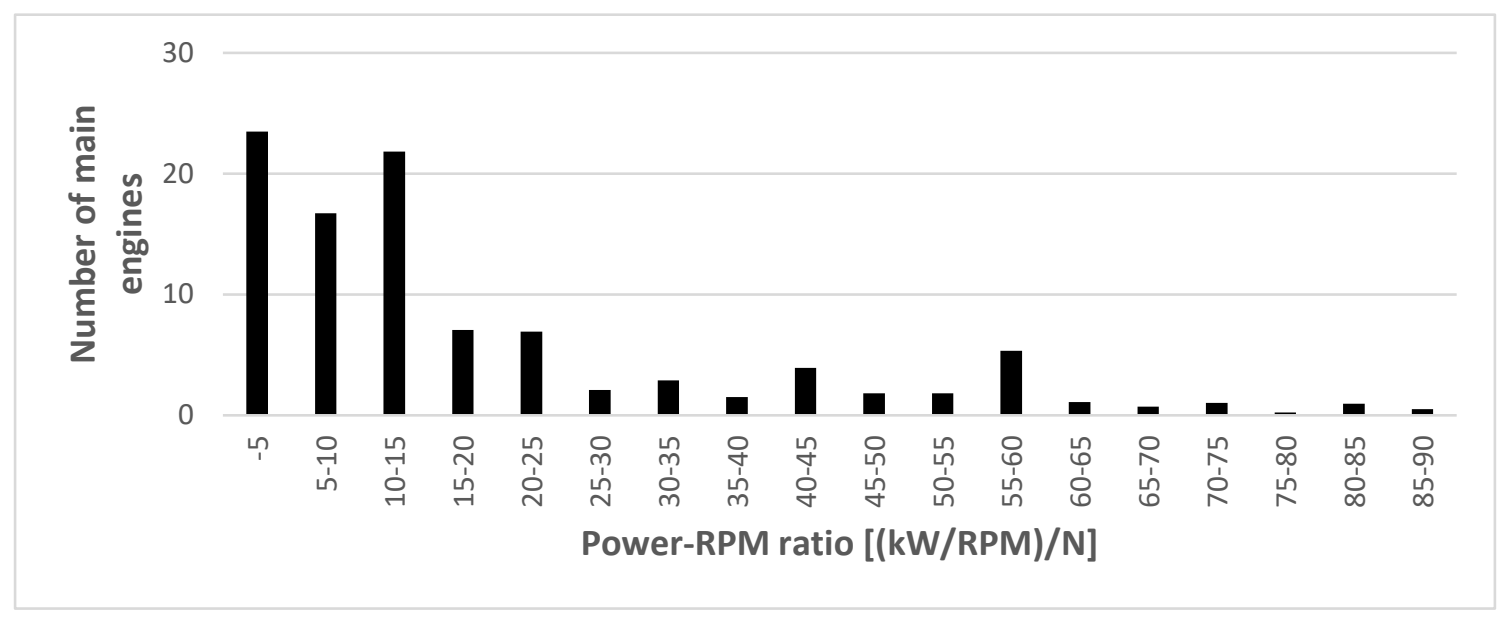

Fig.7 Distribution of power-RPM ratio per cylinder of I-type main engines

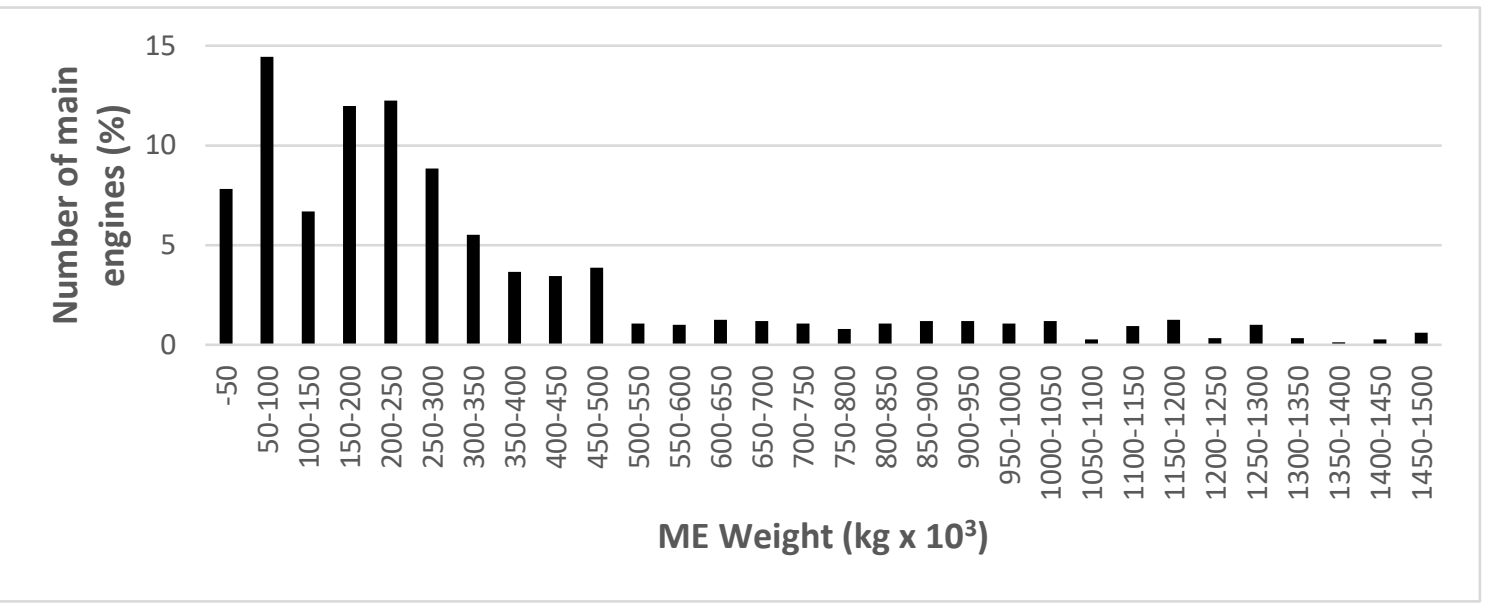

Fig.8 Distribution of ME weight of I-type main engines

\subsection{Power generation machinery weight}

To develop the weight function and verify the assumptions, the data of inline and V-type of a total of 348 auxiliary engines were collected from the product list of different manufacturers. All these engines are in production from time to time.

A general picture of sample auxiliary engines is presented in Tables 3-4 and Figs.9-13. Table 3 summarizes several engines, average RPM, power output, and weight against the cylinder number for $\mathrm{V}$ - and I-type engines. One can easily understand the average RPM, power output and weight of Vand I-type auxiliary engines of same cylinder number. It also appears that these values are far apart for auxiliary engines of a particular cylinder number for V-and I-type.

Table 3 Summary of auxiliary engines by V-type and I-type

\begin{tabular}{|c|c|c|c|c|l|r|r|r|r|}
\hline Serial & Cylinder & \multicolumn{3}{|c|}{ Average for V-type engines } & \multicolumn{4}{|c|}{ Average for I-type engines } \\
\cline { 3 - 10 } No. & number & No. & RPM & Power & Weight & No. & RPM & Power & Weight \\
\hline 1 & 3 & & & & & 2 & 1,800 & 13 & 0.412 \\
\hline 2 & 4 & & & & & 21 & 1,700 & 98 & 1.930 \\
\hline
\end{tabular}




\begin{tabular}{|r|r|r|r|r|r|r|r|r|r|}
3 & 5 & & & & & 10 & 804 & 780 & 21 \\
\hline 4 & 6 & & & & & 120 & 1,102 & 1,174 & 30 \\
\hline 5 & 7 & & & & & 18 & 741 & 2,814 & 75 \\
\hline 6 & 8 & 3 & 1,600 & 742 & 9 & 51 & 757 & 2,846 & 69 \\
\hline 7 & 9 & & & & & 31 & 741 & 4,005 & 91 \\
\hline 8 & 10 & & & & & 3 & 720 & 5,446 & 109 \\
\hline 10 & 12 & 28 & 1,147 & 4,508 & 84 & 4 & 1,254 & 4,131 & 64 \\
\hline 12 & 14 & 2 & 720 & 7,198 & 122 & & & & \\
\hline 14 & 16 & 18 & 784 & 8,379 & 154 & 2 & 707 & 10,241 & 146 \\
\hline 15 & 18 & 3 & 720 & 8,562 & 119 & 2 & 707 & 11,526 & 160 \\
\hline 16 & 20 & 1 & 720 & 11,640 & 172 & 1 & 900 & 6,790 & 85 \\
\hline
\end{tabular}

Table 4 is constructed using Table 3. It shows a comparison of some selected characteristics, such as the weight per unit power output, weight per unit power-RPM ratio and power output per unit cylinder, of V-and I-type auxiliary engines against the cylinder number. In Table 4, weight per unit power output for V-type engine varies from 12-19 and for I-type engine varies from 12-33. Weight per unit power-RPM ratio for V-type engines varies from 10,007-21,373 and for I-type engine varies from 9,814-59,328. Power output per unit cylinder for V-type engines varies from 92-582 and for I-type engines varies from 4-640. It is very clear from the table that the magnitudes of the characteristics mentioned above for V-type and I-type engines having the same cylinder number are far apart from each other. This is probably because of inherent differences between V-and I-type concerning geometrical configuration and design considerations. As such, it is not appropriate to combine both types in the same platform for analysis. Therefore, V-type engines are excluded from analysis in this investigation. Only l-type engines are considered to ensure consistency, uniformity, and reliability in the study results.

Table 4 Summary of characteristics of V-type and I-type auxiliary engines

\begin{tabular}{|c|l|r|r|r|r|r|}
\hline \multirow{2}{*}{$\begin{array}{c}\text { Cylinder } \\
\text { number }\end{array}$} & \multicolumn{2}{|c|}{ Weight/power (kg/kW) } & \multicolumn{2}{c|}{ Weight/power-RPM ratio (kg/T) } & \multicolumn{2}{c|}{ Power/cylinder (kW/N) } \\
\cline { 2 - 7 } & V-type & I-type & V-type & I-type & V-type & \multicolumn{1}{c|}{ I-type } \\
\hline 3 & & 32.960 & & 59,328 & & 4.17 \\
\hline 4 & & 19.694 & & 33,480 & & 24.50 \\
\hline 5 & & 26.923 & & 21,646 & & 156.00 \\
\hline 6 & & 25.276 & & 27,854 & & 195.67 \\
\hline 7 & & 26.652 & & 19,749 & & 402.00 \\
\hline 8 & 12.007 & 24.245 & 19,211 & 18,353 & 92.75 & 355.75 \\
\hline 9 & & 22.722 & & 16,837 & & 445.00 \\
\hline 10 & & 20.076 & & 14,455 & & 544.60 \\
\hline 12 & 18.634 & 15.464 & 21,373 & 19,391 & 375.67 & 344.25 \\
\hline 14 & 16.949 & & 12,203 & & 514.14 & \\
\hline 16 & 18.379 & 14.256 & 14,409 & 10,079 & 523.69 & 640.06 \\
\hline
\end{tabular}




\begin{tabular}{|r|r|r|r|r|r|r|}
18 & 13.899 & 13.882 & 10,007 & 9,814 & 475.67 & 640.33 \\
\hline 20 & 14.777 & 12.518 & 10,639 & 11,267 & 582.00 & 339.50 \\
\hline
\end{tabular}

Figures 9-13 show the distribution of auxiliary engine type, cylinder number, RPM, power output, and weight. These figures also depict the limiting values of the respective parameters. It can be seen from Fig. 9 that $85 \%$ of sample auxiliary engines are of the I-type. Figures 10 demonstrates the distribution of cylinder number with an average value of 7 cylinders and $65 \%$ of sample auxiliary engines are within this cylinder number range. Figure 11 reflects the allocation of engine RPM with an average value of 1001 RPM and 71\% of sample auxiliary engines are within this RPM range. Figure 12 demonstrates the distribution of power output with an average value of $2,036 \mathrm{~kW}$ and $65 \%$ of sample auxiliary engines are within this power output range. Figure 13 reflects the distribution of AE weight with an average value of 47 tonnes and $68 \%$ of sample auxiliary engines are within this $A E$ weight range.

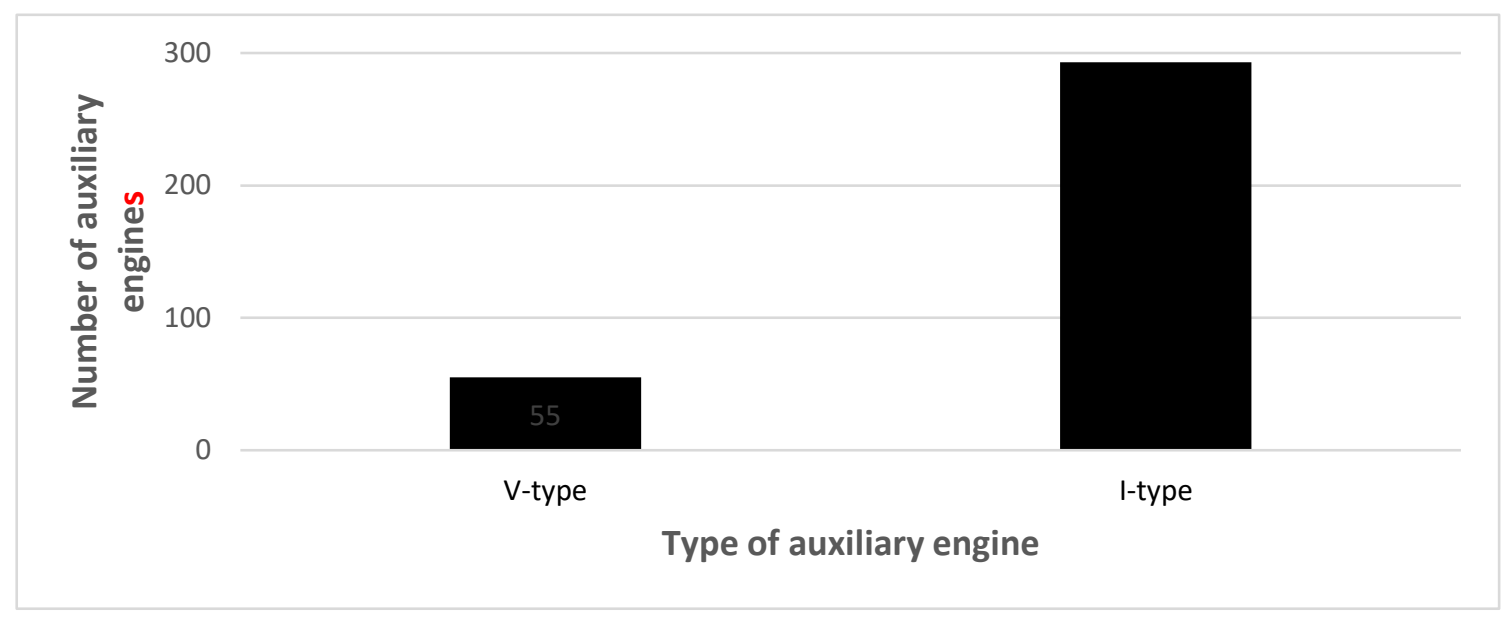

Fig.9 Distribution of type of auxiliary engines

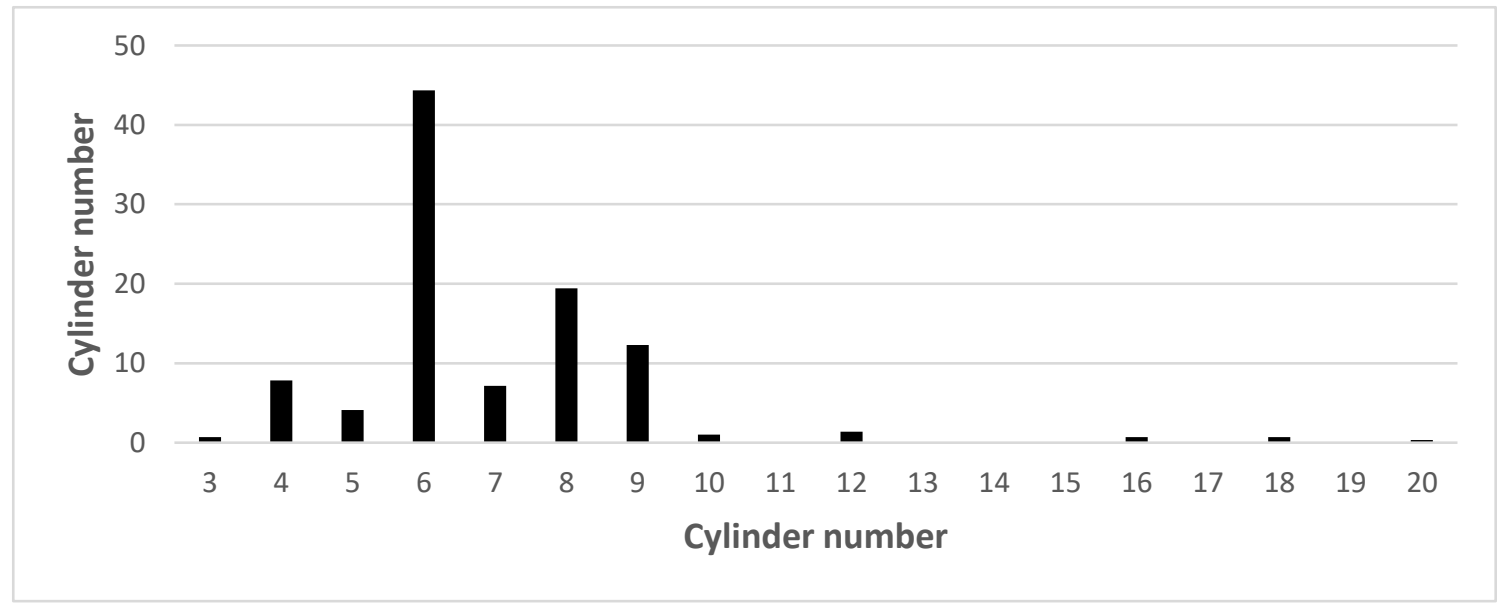

Fig.10 Distribution of cylinder number of I-type auxiliary engines 


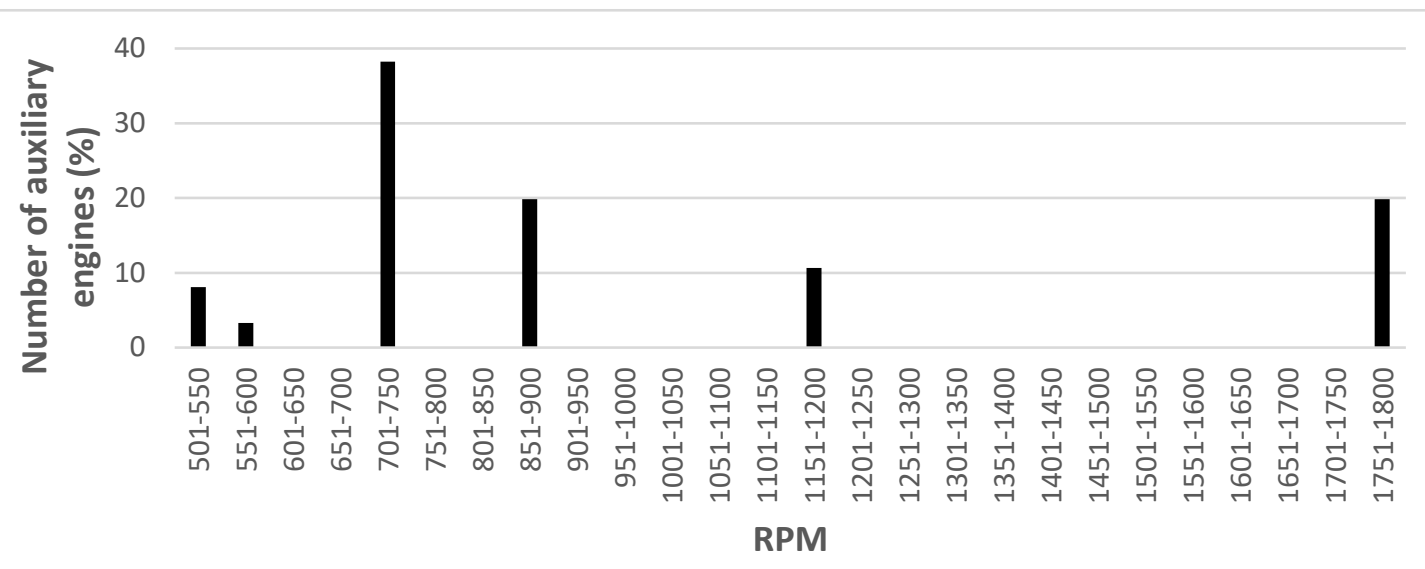

Fig.11 Distribution of RPM of I-type auxiliary engines

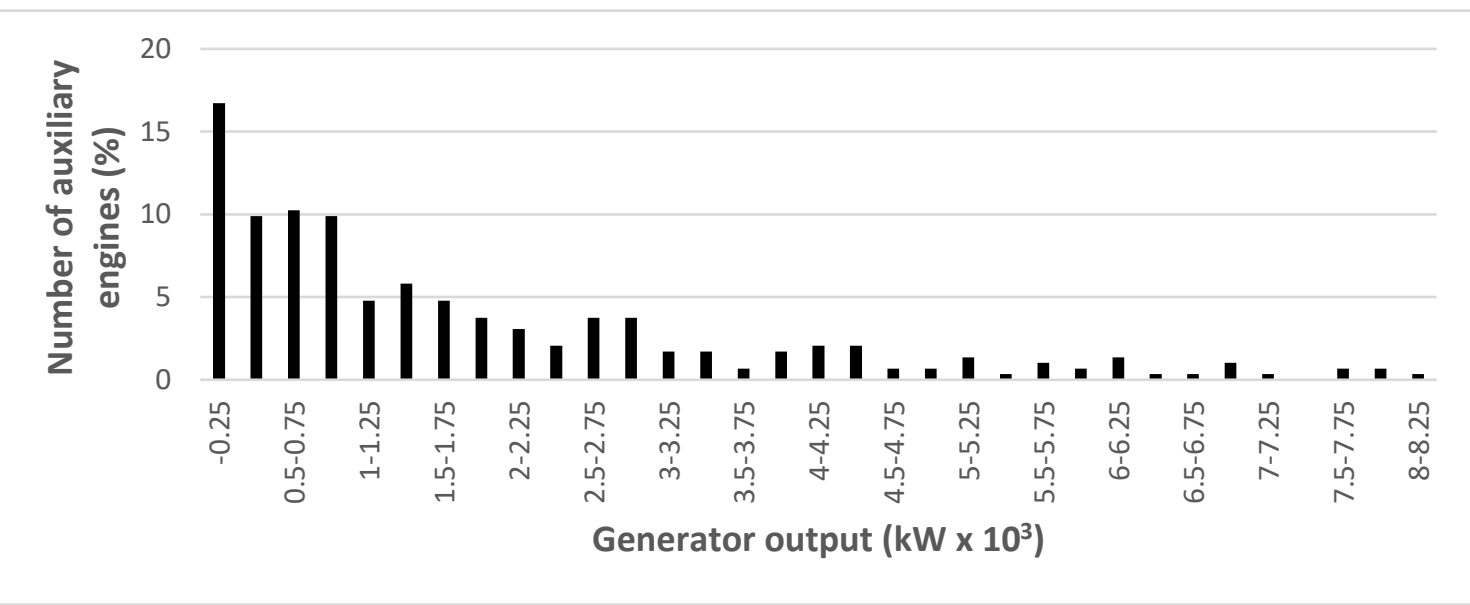

Fig.12 Distribution of power output of I-type auxiliary engines

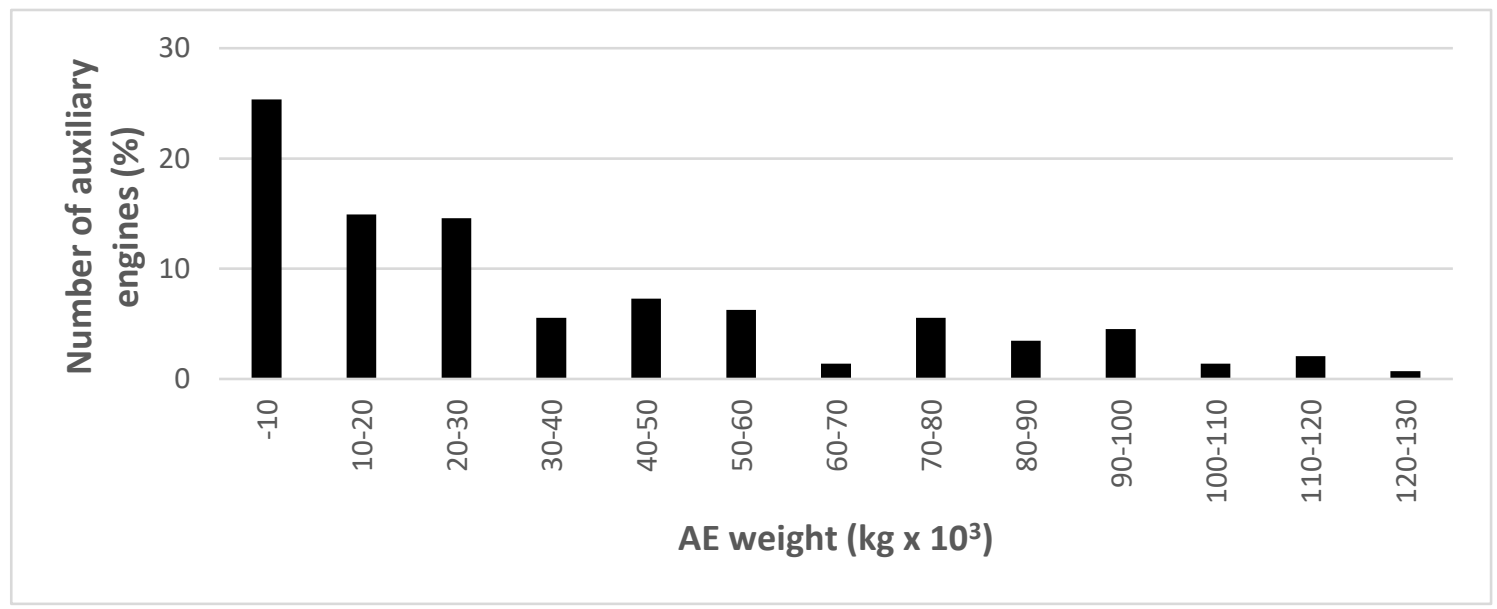

Fig.13 Distribution of AE weight of I-type auxiliary engines

\subsection{Methodology}


To establish the relationship between the main engine weight and auxiliary engine weight and its dependent variables, the following functions of the linear equations 6 and 7 are chosen because individually, the independent variables are linearly associated with the dependent variable as per initial assumptions.

$W_{\mathrm{ME}}=f(P, N, R P M)$

$W_{\mathrm{AE}}=f(P, N, R P M)$

Where $W_{\mathrm{ME}}$ is the main engine weight (tonne $=\mathrm{kg} \times 10^{3}$ )

$W_{\mathrm{AE}}$ is the auxiliary engine weight (tonne $=\mathrm{kg} \times 10^{3}$ )

$P$ is the power output $(\mathrm{kW})$ of the main engine (ME) at $100 \%$ MCR and

electrical power output $(\mathrm{kW})$ of the auxiliary engine $(\mathrm{AE})$

$N$ is the cylinder number of engine

$R P M$ is the speed of the engine (revolution per minute)

\subsection{Data analysis}

\subsection{Marine propulsion machinery weight analysis}

Initial investigations on pairs of interest variables related to main engine weight are shown in Figs.2035. Pairs of variables refer to a combination of the dependent variable, the main engine weight and an independent variable, such as power output, cylinder number, engine RPM, power-RPM ratio and power-RPM ratio per cylinder. Correlation coefficients are calculated for each relationship under different equation forms for comparison purposes and presented in Tables 5-9.

\subsubsection{Engine power output}

Main engine weights are analyzed against the power output to determine the ME weight's behaviour from different viewpoints and presented in Figs.14-15. Figure 14 illustrates the ME weight trend (observed values) against the power output, and it seems to be linearly associated. Figure 15 represents the behaviour of average ME weight (average by engine power output group) against average power output. It also shows the sign of linearity among variables. This depicts that with the rise in power output, the ME weight of the said engine increases linearly. Therefore, it is expected that with the increase of power output, the ME weight would be increased too. This is in line with the first assumption.

Figures are investigated for other forms of relationships, and the results are presented in Table 5. Table 5 shows the correlation coefficients under different equation forms. It suggests that polynomial form (second-order), power form and linear form yield high correlation coefficients for Figs.14 and 15, and differences are minimal. 


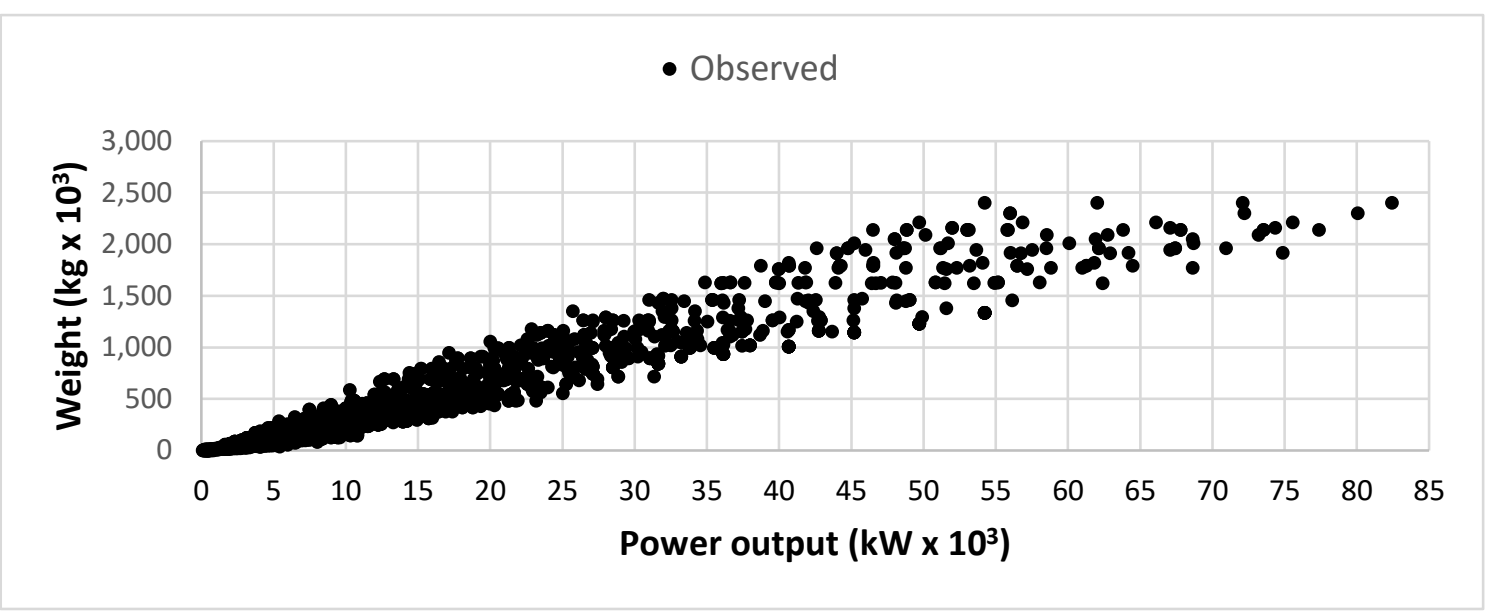

Fig.14 ME weight versus power output of I-type ME

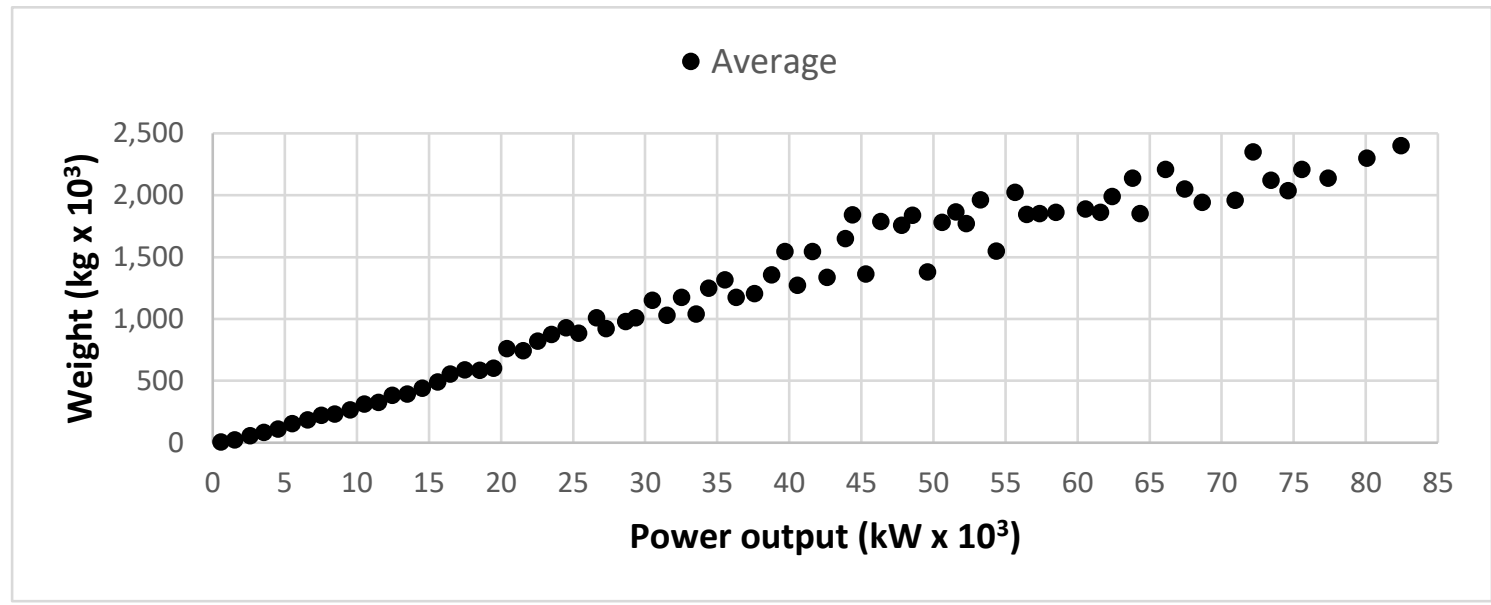

Fig.15 Average ME weight versus average power output of I-type ME

Table 5 Summary of correlation coefficients

\begin{tabular}{|c|c|r|r|r|r|r|r|r|}
\hline \multirow{2}{*}{$\begin{array}{c}\text { Figure } \\
\text { No. }\end{array}$} & \multirow{2}{*}{ Variables } & $y=\mathrm{m}^{*} x+\mathrm{c}$ & $y=\mathrm{m}^{*} x$ & $y=\mathrm{m}^{*} x^{\mathrm{n}}$ & $y=\mathrm{m}^{*} \mathrm{e}^{\mathrm{b} x}$ & $y=\mathrm{a} * x^{2}+\mathrm{b}^{*} x+\mathrm{c}$ & $y=\mathrm{a} * \ln (x)+\mathrm{c}$ \\
\cline { 3 - 8 } & & \multicolumn{7}{|c|}{ Correlation coefficients $\left(r^{2}\right)$} \\
\hline 14 & $W$ vs $P$ & 0.9298 & 0.9207 & 0.9256 & 0.5696 & 0.9330 & 0.6606 \\
\hline 15 & $W$ vs $P$ & 0.9544 & 0.9505 & 0.9798 & 0.6582 & 0.9743 & 0.8105 \\
\hline
\end{tabular}

\subsubsection{Engine RPM}

Main engine weights are analyzed against the RPM to determine the ME weight's behavior from different viewpoints, and the results are presented in Figs.16-17. Figure 16 shows the trend of the ME weight (observed values) against RPM. Figure 17 shows the behavior of average ME weight (average by RPM group) against average RPM. It also shows the sign of power form of relationship among variables. This demonstrates that with the increase of RPM, the ME weight decreases. Therefore, it is expected that with the rise in RPM, the ME weight would be reduced. This is in line with the third assumption, but only in the power form, instead of a linear arrangement of an equation. 
Figures are investigated for other types of relationships, and the results are presented in Table 6. Table 6 shows the correlation coefficients under different equation forms and suggest that power form yields high correlation coefficients for Figs.16 and 17.

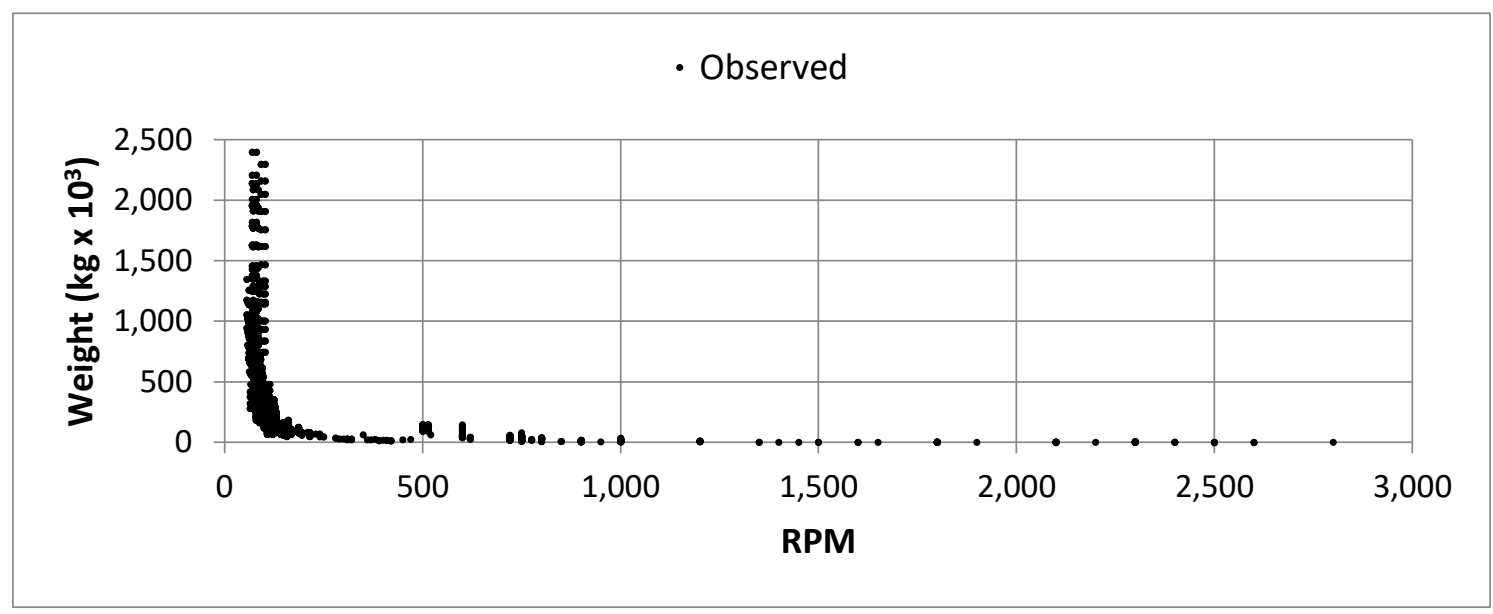

Fig.16 ME weight versus RPM of I-type ME

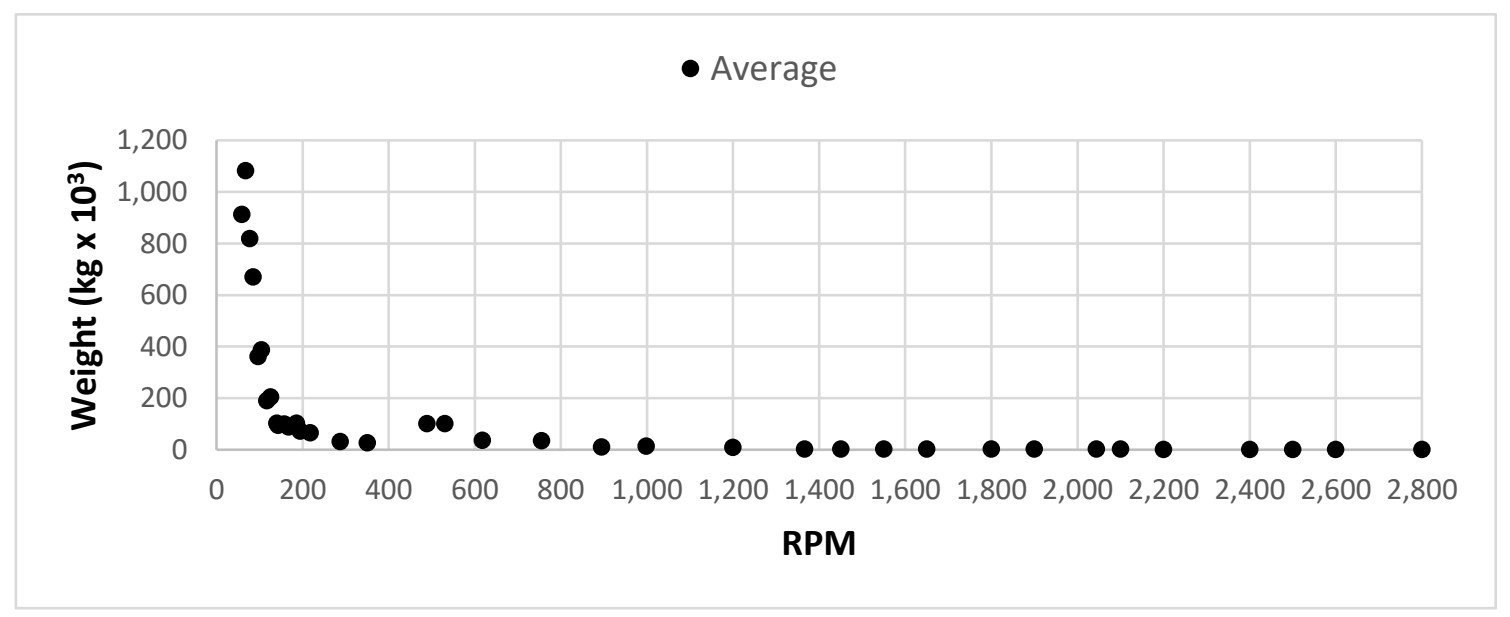

Fig.17 Average ME weight versus average RPM of I-type ME

Table 6 Summary of correlation coefficients

\begin{tabular}{|c|c|c|c|c|c|r|r|r|}
\hline \multirow{2}{*}{$\begin{array}{c}\text { Figure } \\
\text { No. }\end{array}$} & \multirow{2}{*}{ Variables } & $y=\mathrm{m}^{*} x+\mathrm{c}$ & $y=\mathrm{m}^{*} x$ & $y=\mathrm{m}^{*} x^{\mathrm{n}}$ & $y=\mathrm{m}^{*} \mathrm{e}^{\mathrm{bx}}$ & $y=\mathrm{a}^{*} x^{2}+\mathrm{b}^{*} x+\mathrm{c}$ & $y=\mathrm{a} \ln (x)+\mathrm{c}$ \\
\cline { 3 - 8 } & & \multicolumn{7}{|c|}{ Correlation coefficients $\left(r^{2}\right)$} \\
\hline 16 & W vs RPM & $0.0842(-)$ & -0.7330 & 0.7201 & 0.5608 & 0.1414 & 0.2249 \\
\hline 17 & W vs RPM & $0.2600(-)$ & -0.3170 & 0.9317 & 0.8837 & 0.3841 & 0.4974 \\
\hline
\end{tabular}

\subsubsection{Cylinder number}

Main engine weights are analyzed against the cylinder number to determine the ME weight's behaviour from different viewpoints and presented in Figs.18-19. Figure 18 demonstrates the pattern of ME weight (observed value) against the cylinder number. Figures 19 shows the average ME weight 
(average by cylinder number) against the cylinder number. It also shows the sign of linearity among variables. Although the observed values show a full range of ME weight against the cylinder number, this demonstrates that with the rise in the engine's cylinder number, the ME weight of the said engine increases linearly. Therefore, it is expected that with the increase in the cylinder number, the ME weight would be increased too. This is in line with the fourth assumption. Careful investigation of Fig.19 would suggest that the average rate of increase in ME weight per cylinder group are different. A remarkable increase in ME weight is observed from 9 to 10 cylinders and may be considered the transition from the first group to the second group.

Figures are investigated for other forms of relationships, and the results are presented in Table 7. Table 7 shows the correlation coefficients under different equation forms, and it suggests that polynomial form forms yield high correlation coefficients for Figs.18 and 19 .

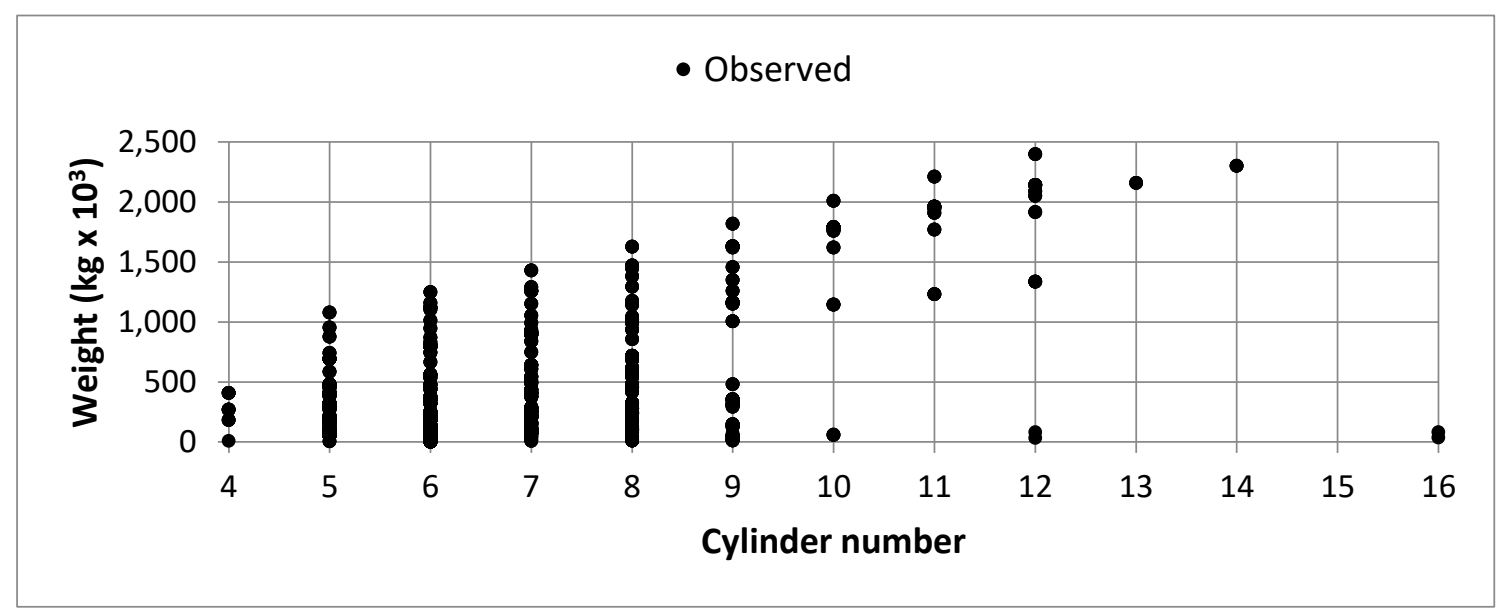

Fig.18 ME weight versus cylinder number of I-type ME

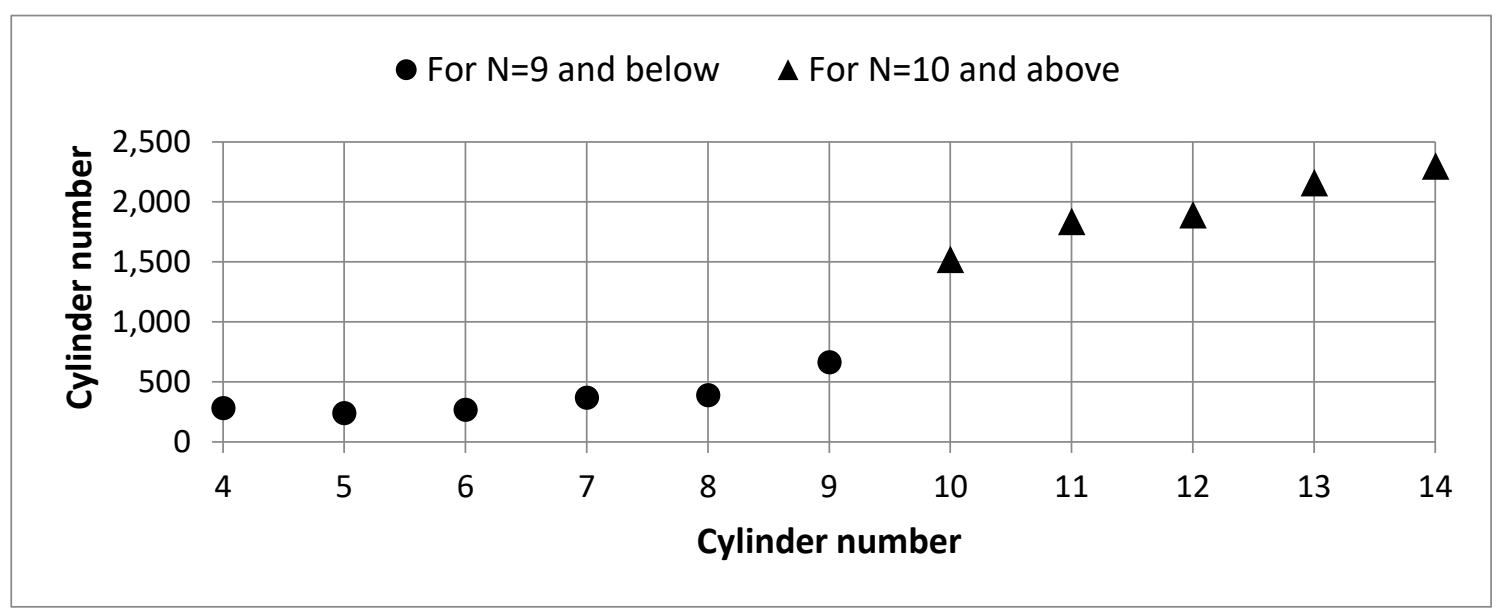

Fig.19 Average ME weight versus cylinder number of I-type ME 
Table 7 Summary of correlation coefficients

\begin{tabular}{|c|c|r|r|r|r|r|r|}
\hline \multirow{2}{*}{$\begin{array}{c}\text { Figure } \\
\text { No. }\end{array}$} & \multirow{2}{*}{ Variables } & $y=\mathrm{m}^{*} x+\mathrm{c}$ & $y=\mathrm{m}^{*} x$ & $y=\mathrm{m}^{*} x^{\mathrm{n}}$ & $y=\mathrm{m}^{*} \mathrm{e}^{\mathrm{bx}}$ & $y=\mathrm{a} * x^{2}+\mathrm{b}^{*} x+\mathrm{c}$ & $y=\mathrm{a} \ln (x)+\mathrm{c}$ \\
\cline { 3 - 8 } & & \multicolumn{7}{|c|}{ Correlation coefficients $\left(r^{2}\right)$} \\
\hline 18 & $W$ vs $N$ & 0.2857 & 0.1770 & 0.0928 & 0.1075 & 0.3608 & 0.2325 \\
\hline 19 & $W$ vs $N$ & 0.3190 & 0.3081 & 0.1387 & 0.0786 & 0.5204 & 0.3276 \\
\hline
\end{tabular}

\subsubsection{Engine power-RPM ratio}

Main engine weights are analyzed against the power-RPM ratio to determine the ME weight's behavior from different viewpoints and presented in Figs.20-21. Figure 20 demonstrates the trend of the ME weight (observed values) against the power-RPM ratio. Figures 21 illustrates the behavior of average ME weight (average by power-RPM ratio group) against average power-RPM ratio. It also shows the sign of linearity among variables. This demonstrates that with the rise in power-RPM ratio, the ME weight increases linearly. Therefore, it is expected that with the increase of power-RPM ratio, the ME weight would be increased too.

Figures are investigated for other forms of relationships, and the results are presented in Table 8. Table 8 shows the correlation coefficients under different equation forms. It suggests that the power form, polynomial form (second-order) and linear form yield high correlation coefficients for Figs.20 and 21 , and that those are very close.

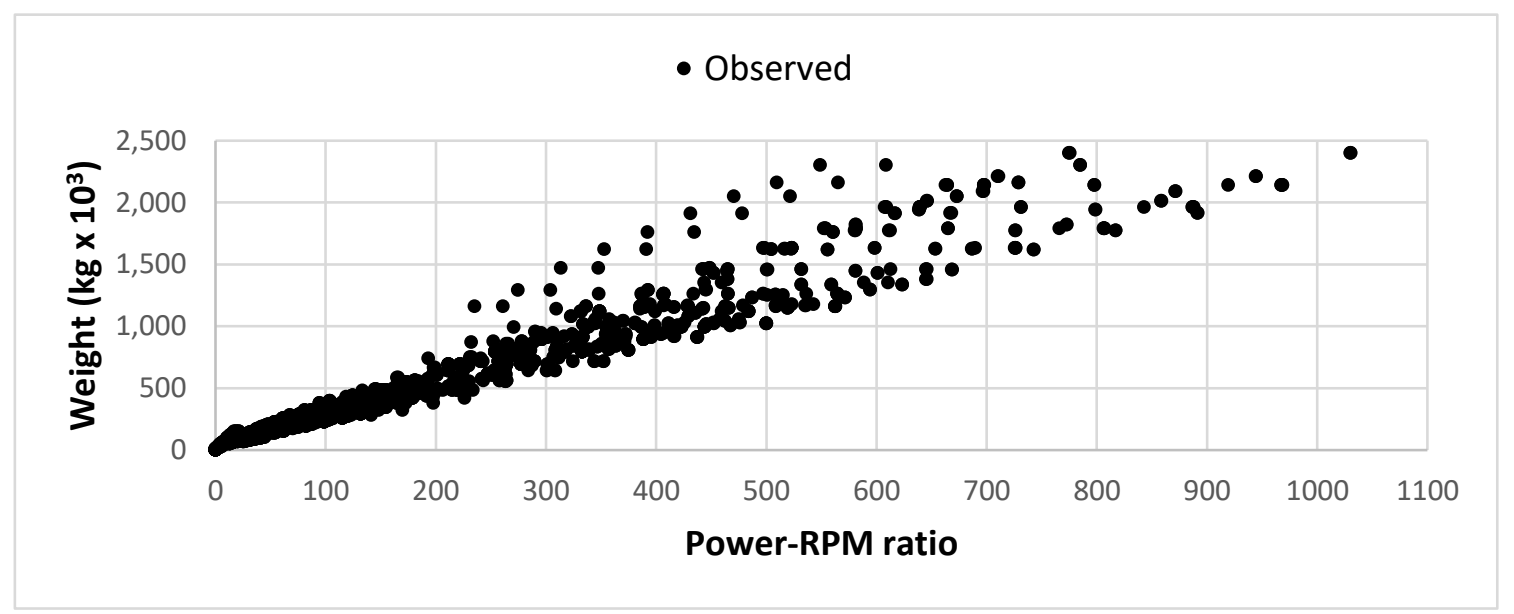

Fig.20 ME weight versus power-RPM ratio of I-type ME 


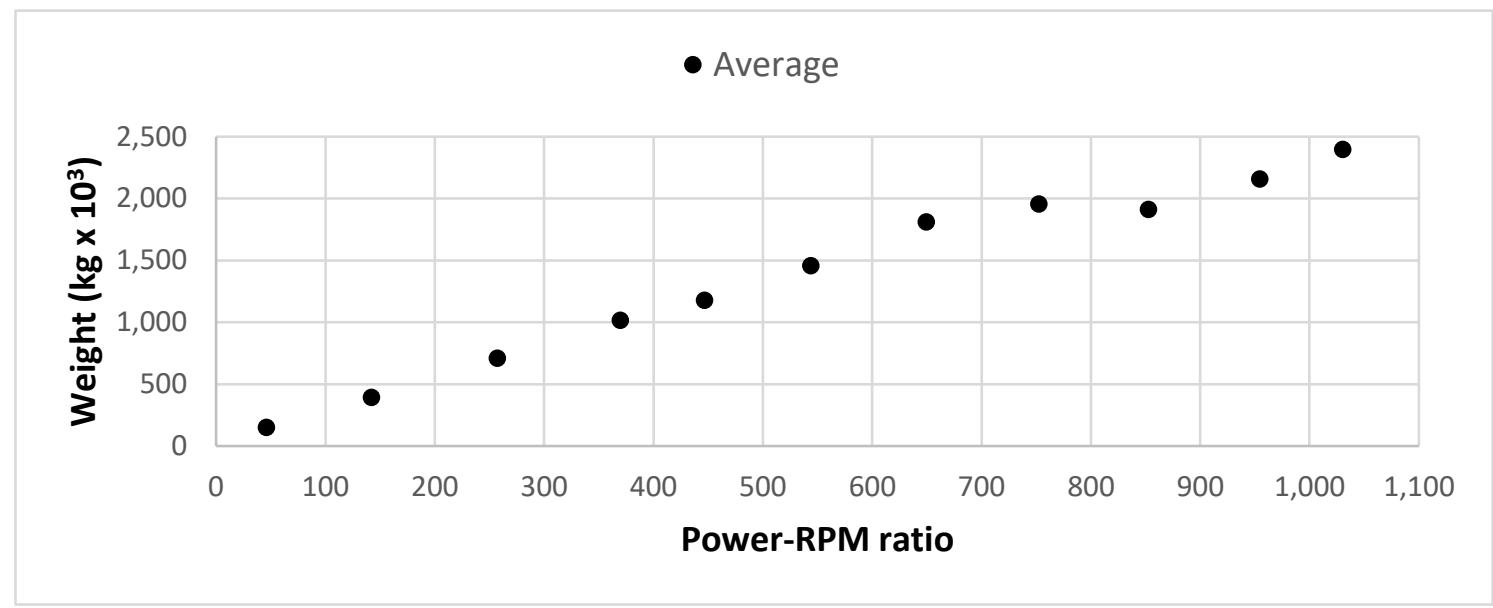

Fig.21 Average ME weight versus average power-RPM ratio of I-type ME

Table 8 Summary of correlation coefficients

\begin{tabular}{|c|c|r|r|r|r|r|r|}
\hline \multirow{2}{*}{$\begin{array}{c}\text { Figure } \\
\text { No. }\end{array}$} & \multirow{2}{*}{ Variables } & $y=\mathrm{m}^{*} x+\mathrm{c}$ & $y=\mathrm{m}^{*} x$ & $y=\mathrm{m}^{*} x^{\mathrm{n}}$ & $y=\mathrm{m}^{*} \mathrm{e}^{\mathrm{b} x}$ & $y=\mathrm{a}^{*} x^{2}+\mathrm{b}^{*} x+\mathrm{c}$ & $y=\mathrm{a} * \ln (x)+\mathrm{c}$ \\
\cline { 3 - 8 } & & \multicolumn{7}{|c|}{ Correlation coefficients $\left(r^{2}\right)$} \\
\hline 20 & Wv $T$ & 0.9518 & 0.9479 & 0.9681 & 0.5737 & 0.9538 & 0.5173 \\
\hline 21 & $W$ vs $T$ & 0.9781 & 0.9679 & 0.9956 & 0.8217 & 0.9938 & 0.8972 \\
\hline
\end{tabular}

\subsubsection{Engine power-RPM ratio per cylinder}

Main engine weights are analyzed against the power-RPM ratio per cylinder to determine the ME weight's behaviour from different viewpoints and presented in Figs.22-23. Figure 22 demonstrates the ME weight (observed values) trend against the power-RPM ratio per cylinder. Figures 23 illustrates the behaviour of average ME weight (average by power-RPM ratio per cylinder group) against average power-RPM ratio per cylinder. It also shows the sign of linearity among variables. This demonstrates that with the rise in power-RPM ratio per cylinder, the ME weight increases linearly. Therefore, it is expected that with the increase of power-RPM ratio per cylinder, the ME weight would be increased too.

Figures are investigated for other forms of relationships, and the results are presented in Table 9. Table 9 shows the correlation coefficients under different equation forms, and it suggests that power form yields high correlation coefficients for Figs.22 and 23. 


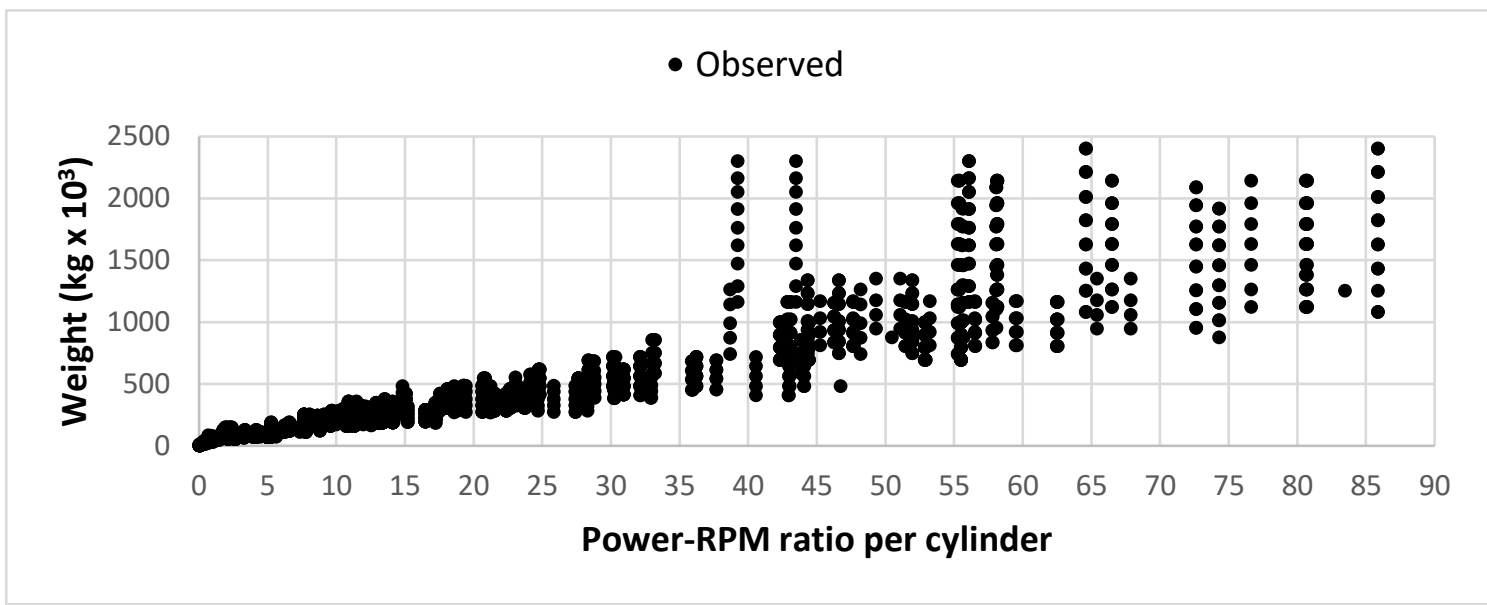

Fig.22 ME weight versus power-RPM ratio per cylinder of I-type ME

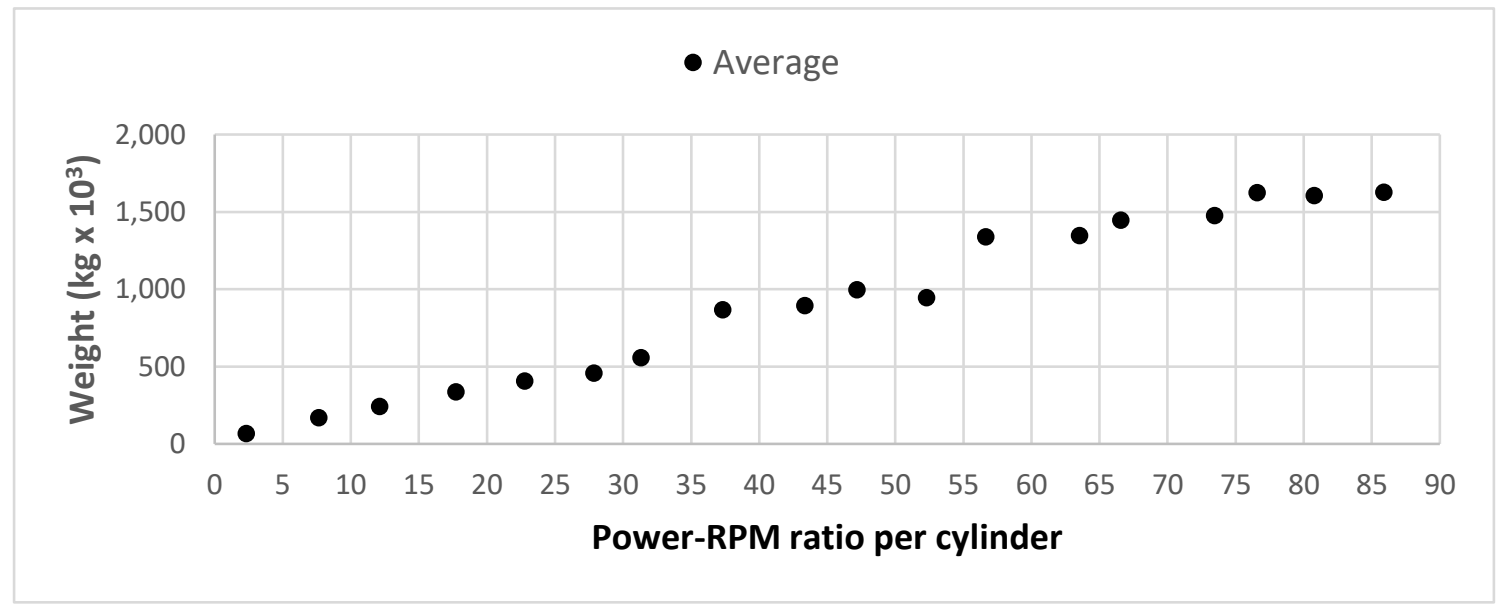

Fig.23 Average ME weight versus average power-RPM ratio per cylinder of I-type ME

Table 9 Summary of correlation coefficients

\begin{tabular}{|c|l|r|r|r|r|r|r|r|}
\hline \multirow{2}{*}{$\begin{array}{c}\text { Figure } \\
\text { No. }\end{array}$} & \multirow{2}{*}{ Variables } & $y=\mathrm{m}^{*} x+\mathrm{c}$ & $y=\mathrm{m}^{*} x$ & $y=\mathrm{m}^{*} x^{\mathrm{n}}$ & $y=\mathrm{m}^{*} \mathrm{e}^{\mathrm{bx}}$ & $y=\mathrm{a} * x^{2}+\mathrm{b}^{*} x+\mathrm{c}$ & $y=\mathrm{a} \ln (x)+\mathrm{c}$ \\
\cline { 3 - 8 } & & \multicolumn{7}{|c|}{ Correlation coefficients $\left(r^{2}\right)$} \\
\hline 22 & $W$ vs $T / N$ & 0.8420 & 0.8419 & 0.9360 & 0.636 & 0.8432 & 0.4489 \\
\hline 23 & $W$ vs $T / N$ & 0.9774 & 0.9770 & 0.9838 & 0.8593 & 0.9783 & 0.7939 \\
\hline
\end{tabular}

At a glance, Table 10 is the summary of Tables 5-9. It shows an overview of the highest correlation coefficients of different relationships under different equation forms. It is clear from the table that ME weight versus power output (Fig.14), ME weight versus cylinder number (Fig.18), and average ME weight versus cylinder number (Fig.19) follow the polynomial form (second-order) of an equation. Rest of relationships follow the power form of an equation. Also, based on $r^{2}$ values, out of ten, seven relationships support the power equation form. Hence, it is unbiased to consider a power form equation for all correlations. 
Table 10 Summary of highest correlation coefficients

\begin{tabular}{|c|c|c|c|c|c|c|c|c|}
\hline \multirow{2}{*}{$\begin{array}{c}\text { Table } \\
\text { No. }\end{array}$} & \multirow{2}{*}{$\begin{array}{c}\text { Figure } \\
\text { No. }\end{array}$} & \multirow{2}{*}{ Variables } & $y=m * x+c$ & $y=m * x$ & $y=m^{*} x^{n}$ & $y=m^{*} e^{b x}$ & $y=a * x^{2}+b * x+c$ & $y=a * \ln (x)+c$ \\
\hline & & & \multicolumn{6}{|c|}{ Correlation coefficients $\left(r^{2}\right)$} \\
\hline \multirow{2}{*}{5} & 14 & $W$ vs $P$ & & & & & 0.9330 & \\
\hline & 15 & $W$ vs $P$ & & & 0.9798 & & & \\
\hline \multirow{2}{*}{6} & 16 & $W$ vs $R P M$ & & & 0.7201 & & & \\
\hline & 17 & $W$ vs $R P M$ & & & 0.9317 & & & \\
\hline \multirow{2}{*}{7} & 18 & $W$ vs $N$ & & & & & 0.3608 & \\
\hline & 19 & $W$ vs $N$ & & & & & 0.5204 & \\
\hline \multirow{2}{*}{8} & 20 & $W$ vs $T$ & & & 0.9681 & & & \\
\hline & 21 & $W$ vs $T$ & & & 0.9955 & & & \\
\hline \multirow{2}{*}{9} & 22 & $W$ vs $T / N$ & & & 0.9360 & & & \\
\hline & 23 & $W$ vs $T / N$ & & & 0.9838 & & & \\
\hline
\end{tabular}

\subsubsection{Weight analysis by speed groups}

In Sub-Sub-Section 3.2.3, it is explained how the RPM of an engine affects the engine weight. In real life, all high-power output engines are of low-speed group engines, and they are cumbersome in weight (for example, 80,080 kW, 102 RPM and 2,300 tonnes, Model - RT-fles96C, version B, Wartsila). Also, all high-speed engines are of low power output, and they are comparatively very light in weight (for example, 599 kW, 2100 RPM and 2 tonnes, Model - C18ACERT, Caterpillar). In between, there is another group called medium-speed (for example, 6,600 kW, 600 RPM and 80 tonnes, Model - 12DKM36 , Daihatsu). Because of the full range of operating speed and hence the torque, the design criterion is also different, and it prompted the analysis of the engine weight under various speed groups. In the following sections, engine weights are examined under three speed groups - low, medium, and high. Usually, from an operational point of view, main engines are classified according to their design speed (revolution per minute, RPM). They are low-, medium- and high-speed. Wartsila Diesel, one of the leading engine manufacturers, defines low-, medium- and high-speed as RPM 400 and below, 401 to 1000 and more than 1000. In this research, the same specification is followed.

\subsubsection{Low-speed group}

Main engine weights under low-speed group (speed $\leq 400$ RPM) are analyzed against the power output, RPM, cylinder number, power-speed ratio and power-speed ratio per cylinder to determine the ME weight's behaviour from different viewpoints and presented in Figs.24-33. Their trends are almost identical to that of Figs.14-23, but with different responses and correlation coefficients. Figures are also investigated for other types of relationships, and the results are presented in Table 11. 
Low-Speed: Observed

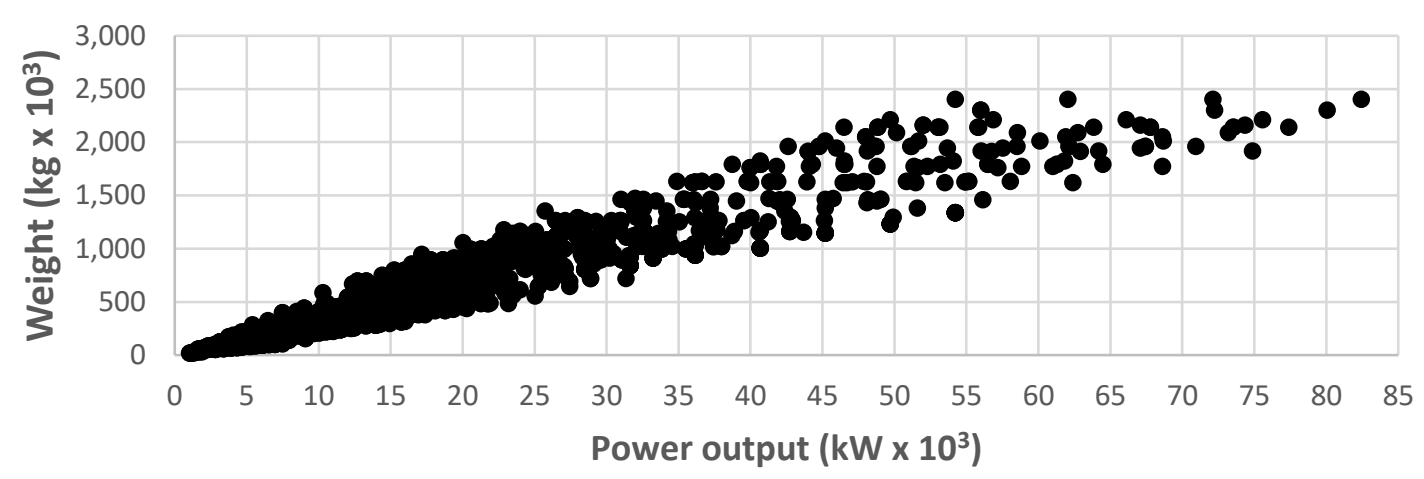

Fig.24 ME weight versus power output of I-type ME (low-speed)

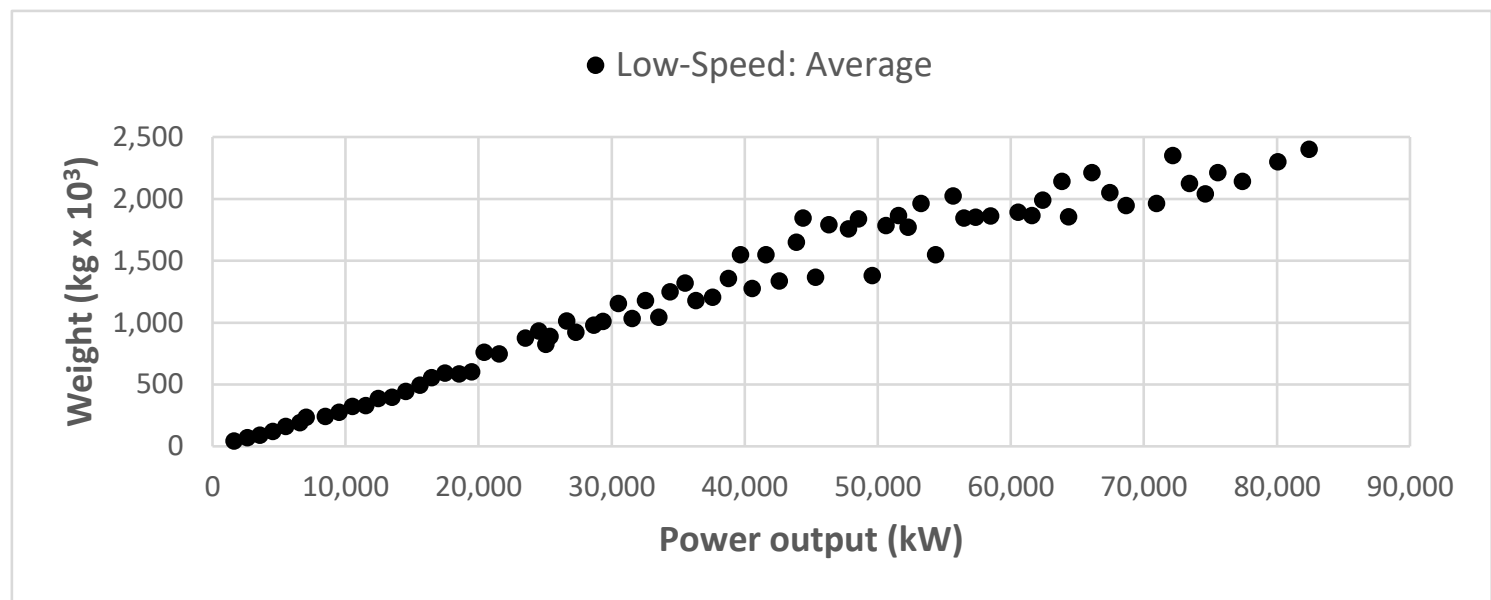

Fig.25 Average ME weight versus average power output of I-type ME (low-speed)

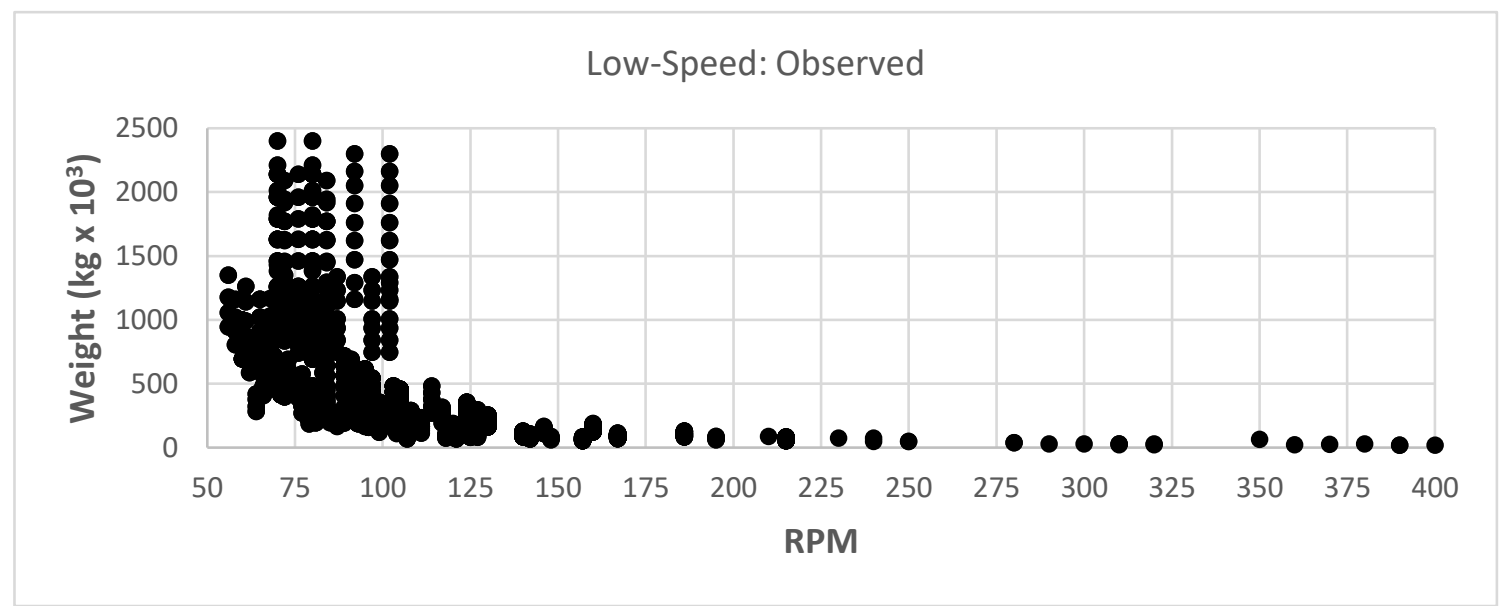

Fig.26 ME weight versus RPM of I-type ME (low-speed) 


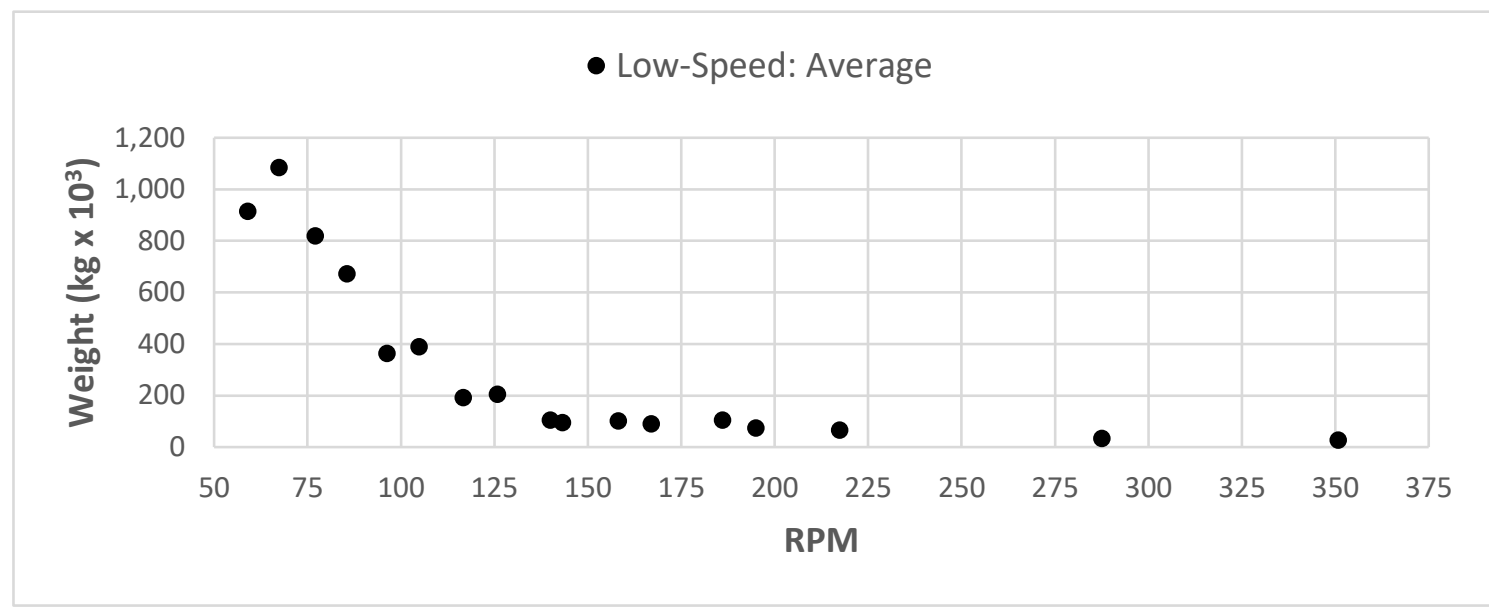

Fig.27 Average ME weight versus average RPM of I-type ME (low-speed)

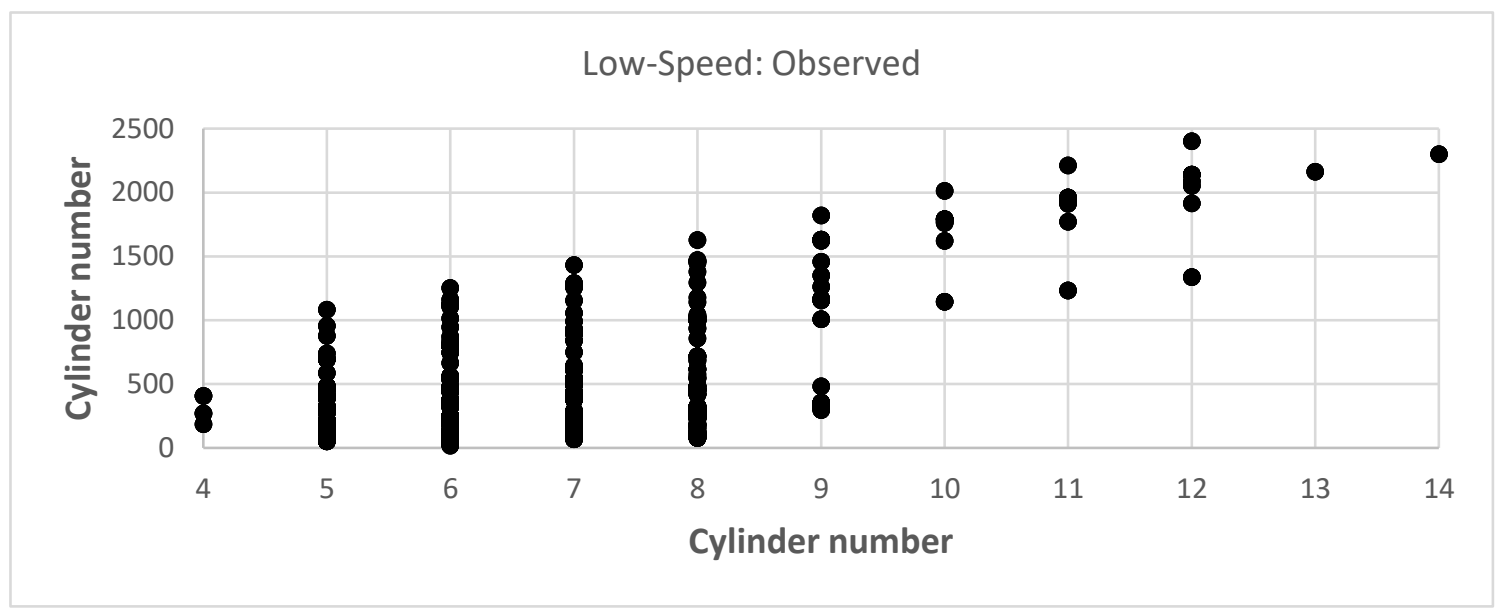

Fig.28 ME Weight versus cylinder number of I-type ME (low-speed)

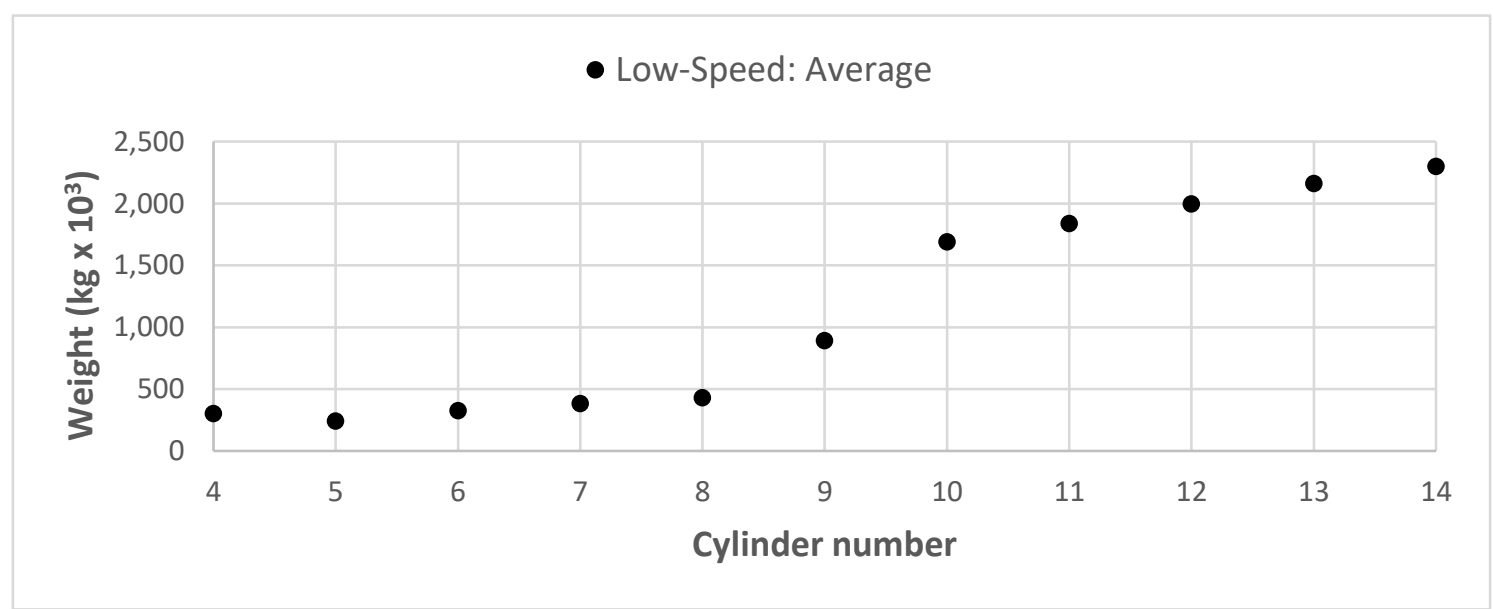

Fig.29 Average ME weight versus cylinder number of I-type ME (low-speed) 
Low-Speed: Observed

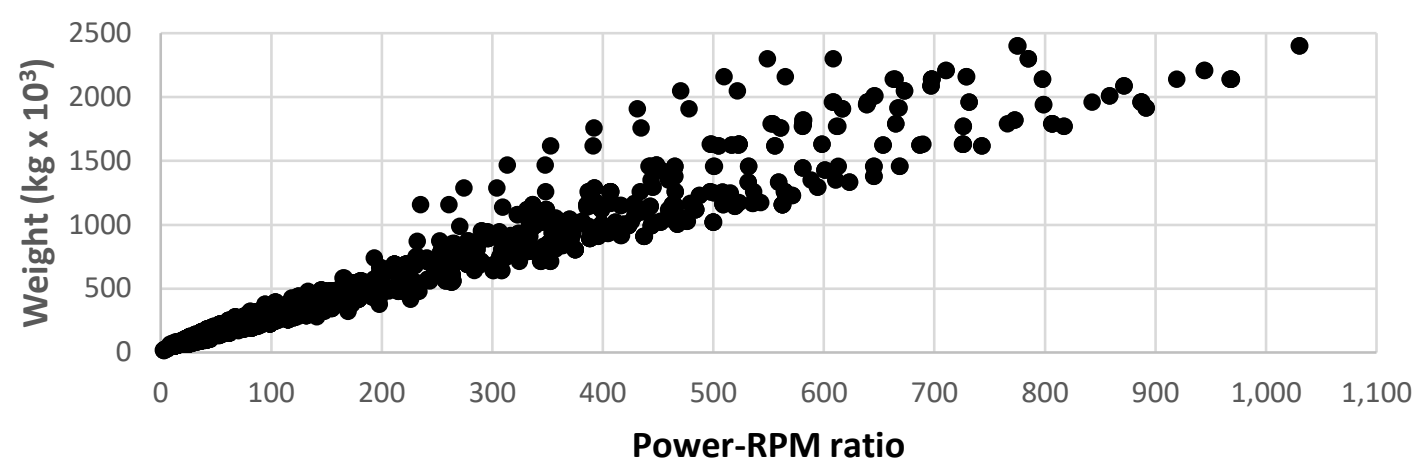

Fig.30 ME weight versus power-RPM ratio of I-type ME (low-speed)

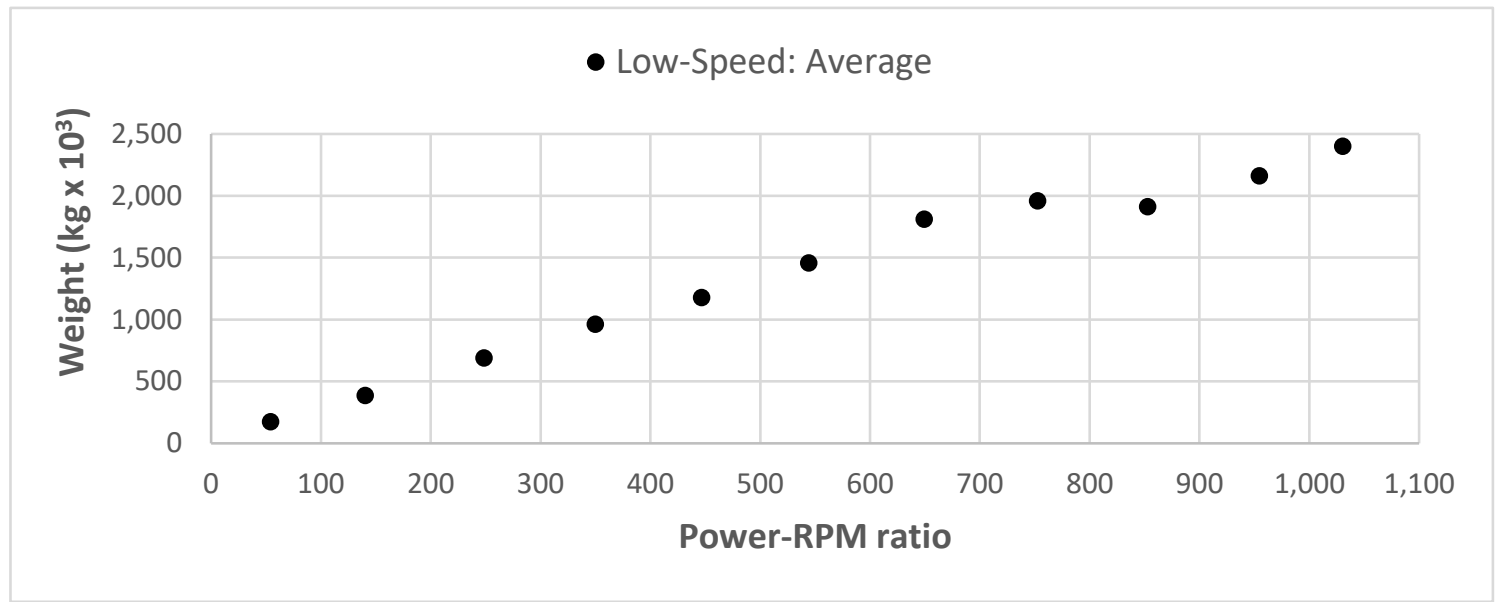

Fig.31 Average ME weight versus average power-RPM ratio of I-type ME (low-speed)

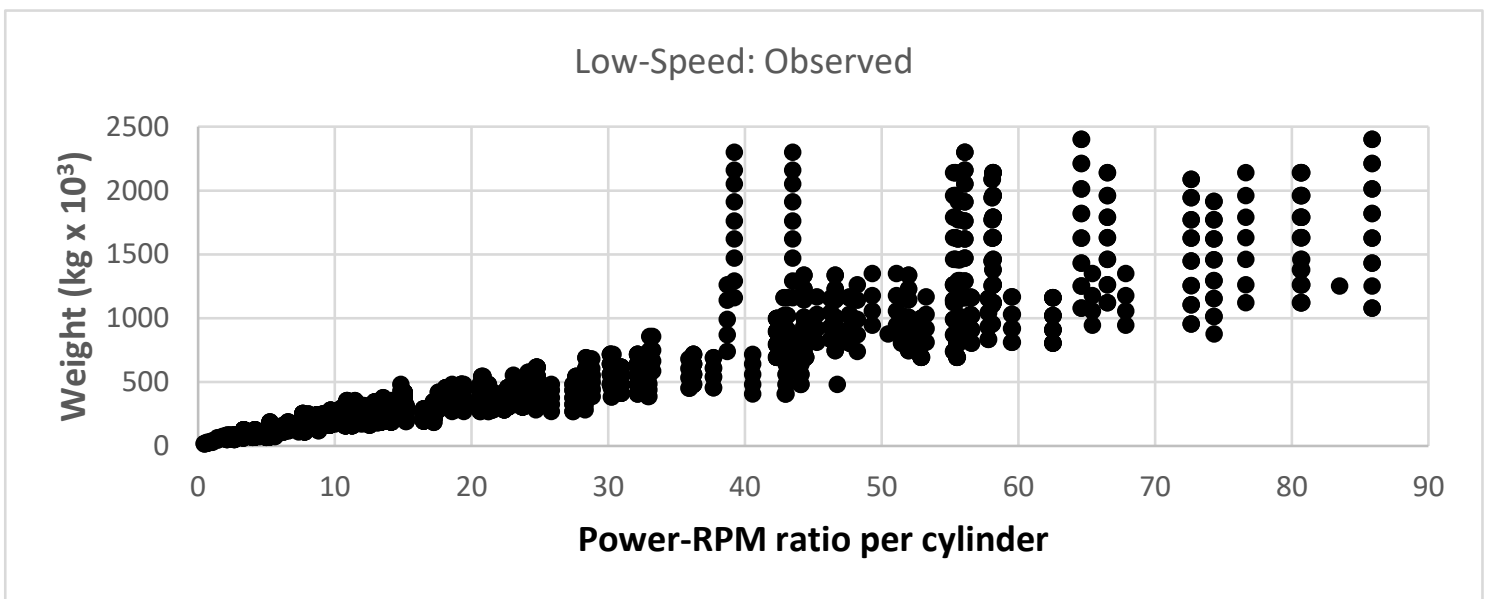

Fig.32 ME weight versus power-RPM ratio per cylinder of I-type ME (low-speed) 


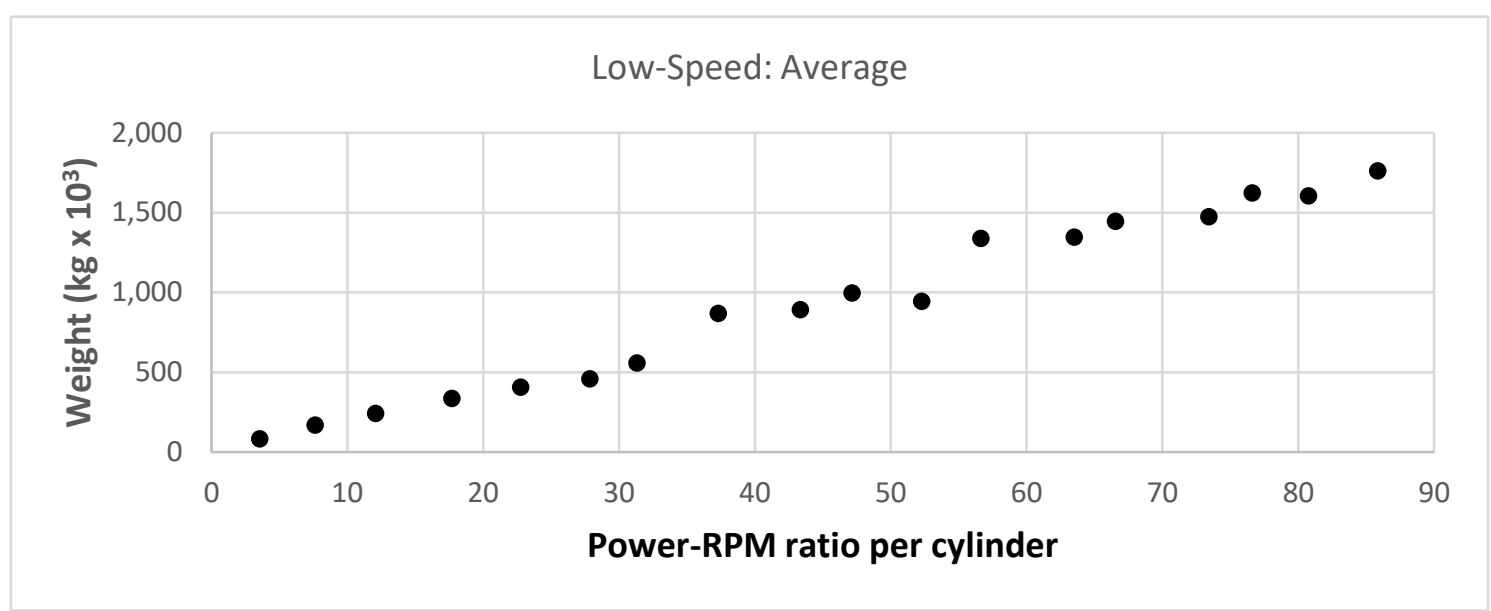

Fig.33 Average ME weight versus average power-RPM ratio per cylinder of I-type ME (low-speed)

Table 11 Summary of correlation coefficients (for low-speed engines)

\begin{tabular}{|c|c|c|c|c|c|c|c|}
\hline \multirow{2}{*}{$\begin{array}{c}\text { Figure } \\
\text { No. }\end{array}$} & \multirow{2}{*}{ Variables } & $y=m^{*} x+c$ & $y=m^{*} x$ & $y=m^{*} x^{n}$ & $y=m * e^{b x}$ & $y=a * x^{2}+b * x+c$ & $y=a * \ln (x)+c$ \\
\hline & & \multicolumn{6}{|c|}{ Correlation coefficients $\left(r^{2}\right)$} \\
\hline 24 & \multirow{2}{*}{$W$ vs $P$} & 0.9268 & 0.9252 & 0.9304 & 0.7384 & 0.9305 & 0.7693 \\
\hline 25 & & 0.9531 & 0.9484 & 0.9859 & 0.7584 & 0.9730 & 0.8635 \\
\hline 26 & \multirow{2}{*}{$W$ vs $R P M$} & $0.2760(-)$ & -0.3720 & 0.6543 & 0.5855 & 0.3749 & 0.3591 \\
\hline 27 & & $0.5190(-)$ & -0.6180 & 0.9518 & 0.8338 & 0.8548 & 0.7486 \\
\hline 28 & \multirow{2}{*}{$W$ vs $N$} & 0.3620 & 0.2191 & 0.2095 & 0.2379 & 0.4820 & 0.2941 \\
\hline 29 & & 0.9073 & 0.7207 & 0.8767 & 0.9109 & 0.9229 & 0.8275 \\
\hline 30 & \multirow{2}{*}{$W$ vs $T$} & 0.9485 & 0.9447 & 0.9699 & 0.7633 & 0.9507 & 0.7322 \\
\hline 31 & & 0.9777 & 0.9671 & 0.9952 & 0.8335 & 0.9896 & 0.9017 \\
\hline 32 & \multirow{2}{*}{$W$ vs $T / N$} & 0.8309 & 0.8305 & 0.9243 & 0.8101 & 0.8317 & 0.6436 \\
\hline 33 & & 0.9822 & 0.9816 & 0.9870 & 0.8802 & 0.9822 & 0.8270 \\
\hline
\end{tabular}

\subsubsection{Medium-speed group}

Main engine weights under medium-speed group ( $400<$ speed $\leq 1000$ RPM) are analyzed against the power output, RPM, cylinder number, power-speed ratio and power-speed ratio per cylinder to determine the ME's behaviour weight from different viewpoints and presented in Figs.34-43. Their trends are almost identical to that of Figs.14-23 but with different responses and correlation coefficients. Figures are also investigated for other types of relationships, and the results are presented in Table 12. 


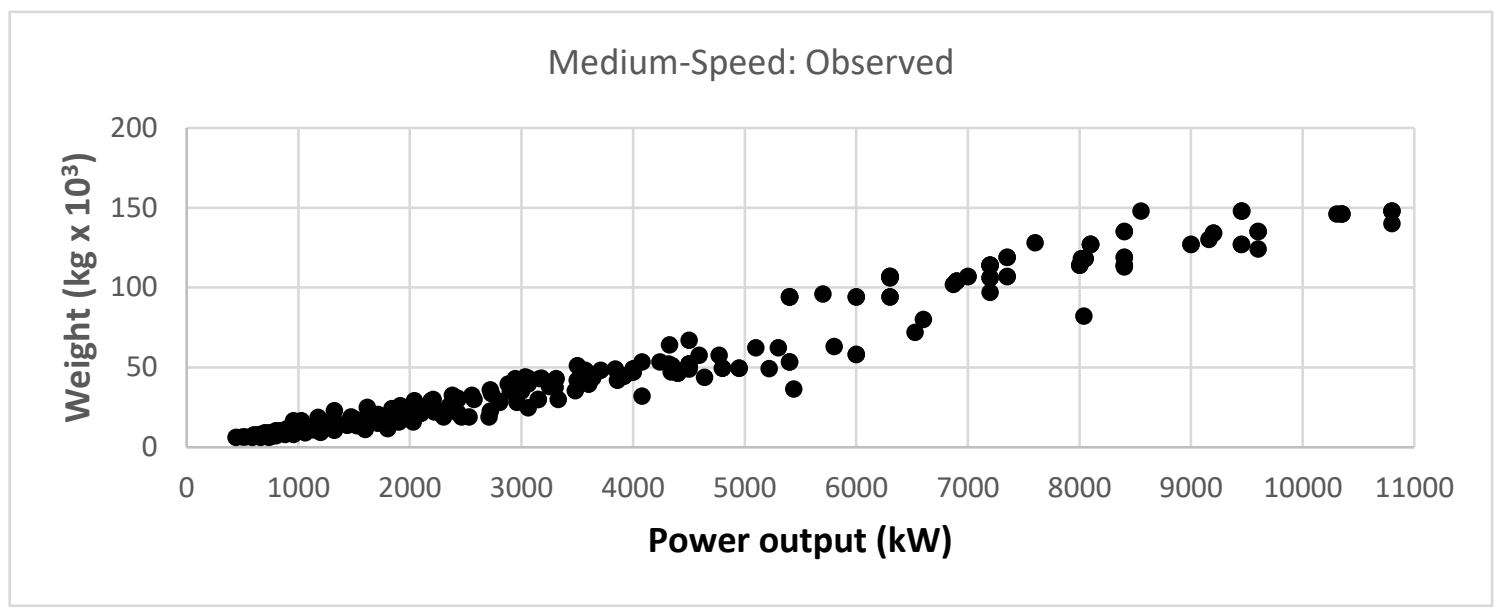

Fig.34 ME weight versus power output of I-type ME (medium-speed)

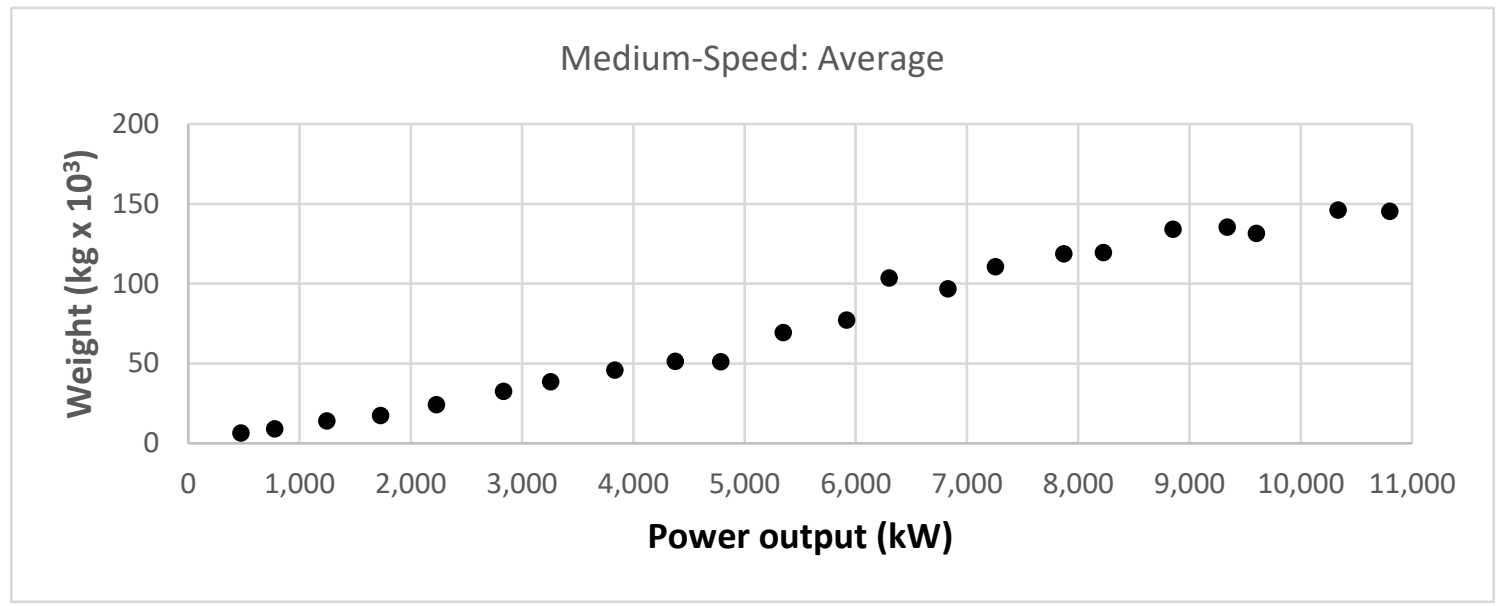

Fig.35 Average ME weight versus average power output of I-type ME (medium-speed)

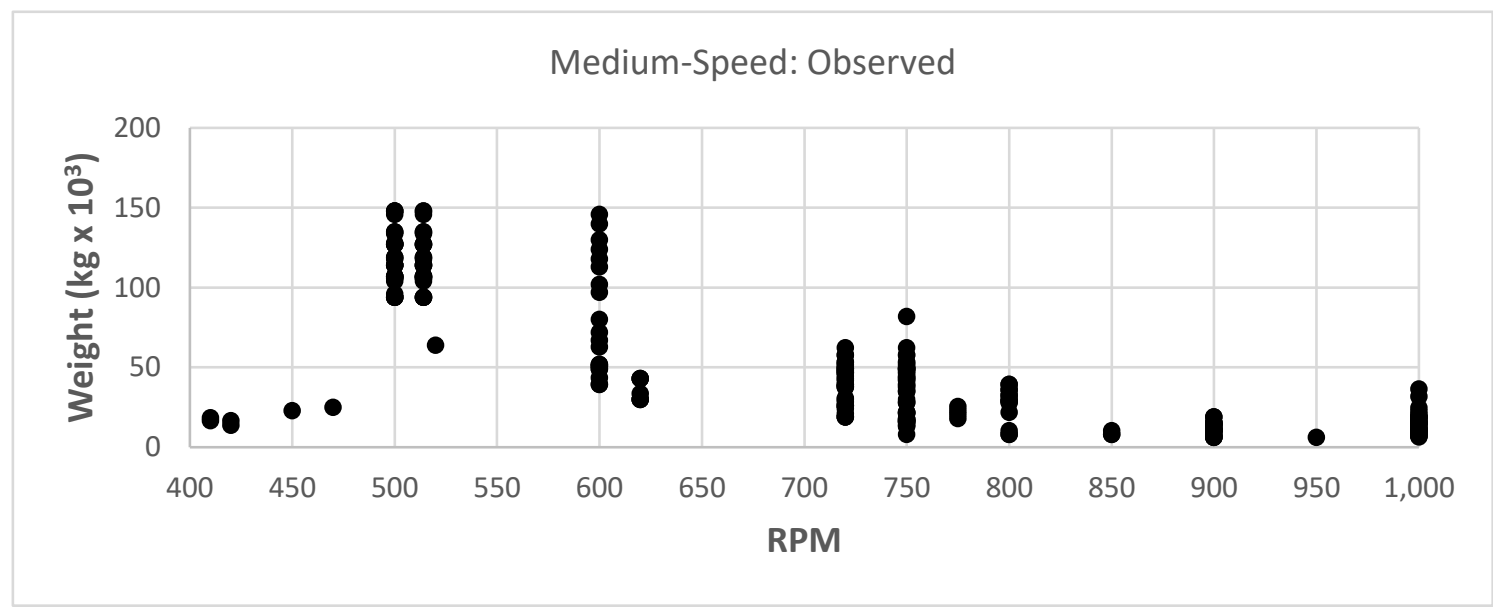

Fig.36 ME weight versus RPM of I-type ME (medium-speed) 


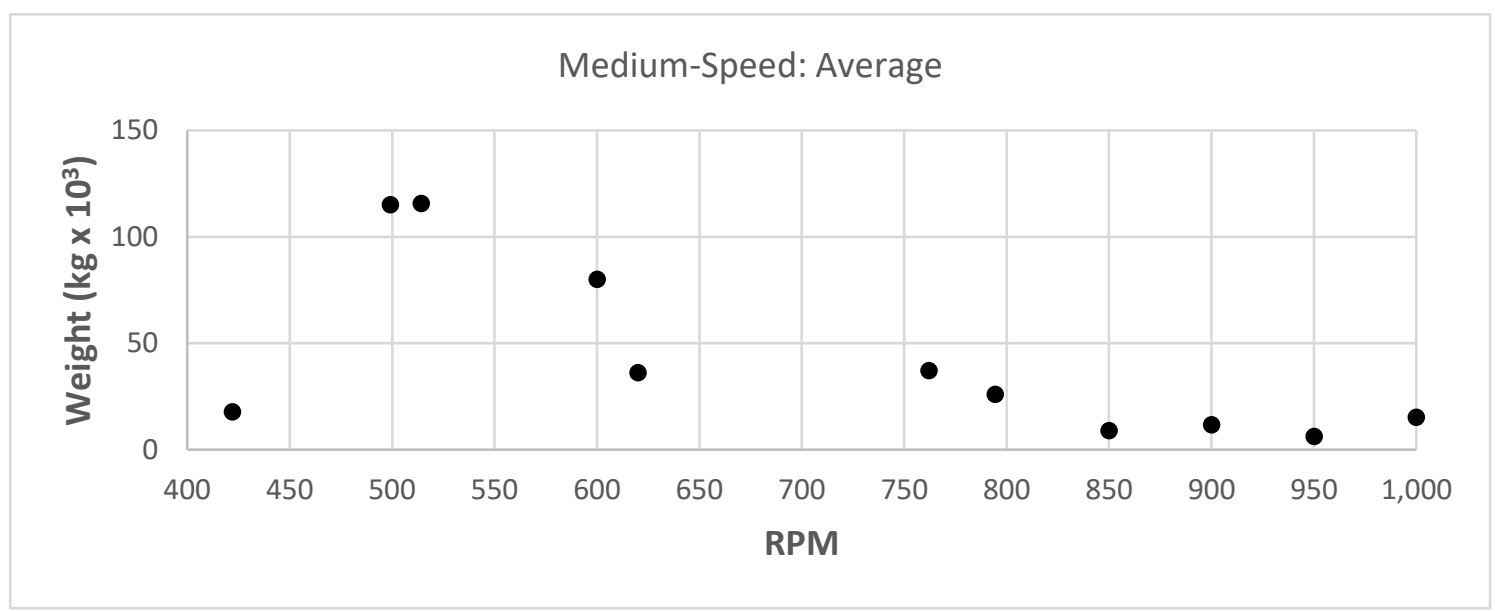

Fig.37 Average ME weight versus average RPM of I-type ME (medium-speed)

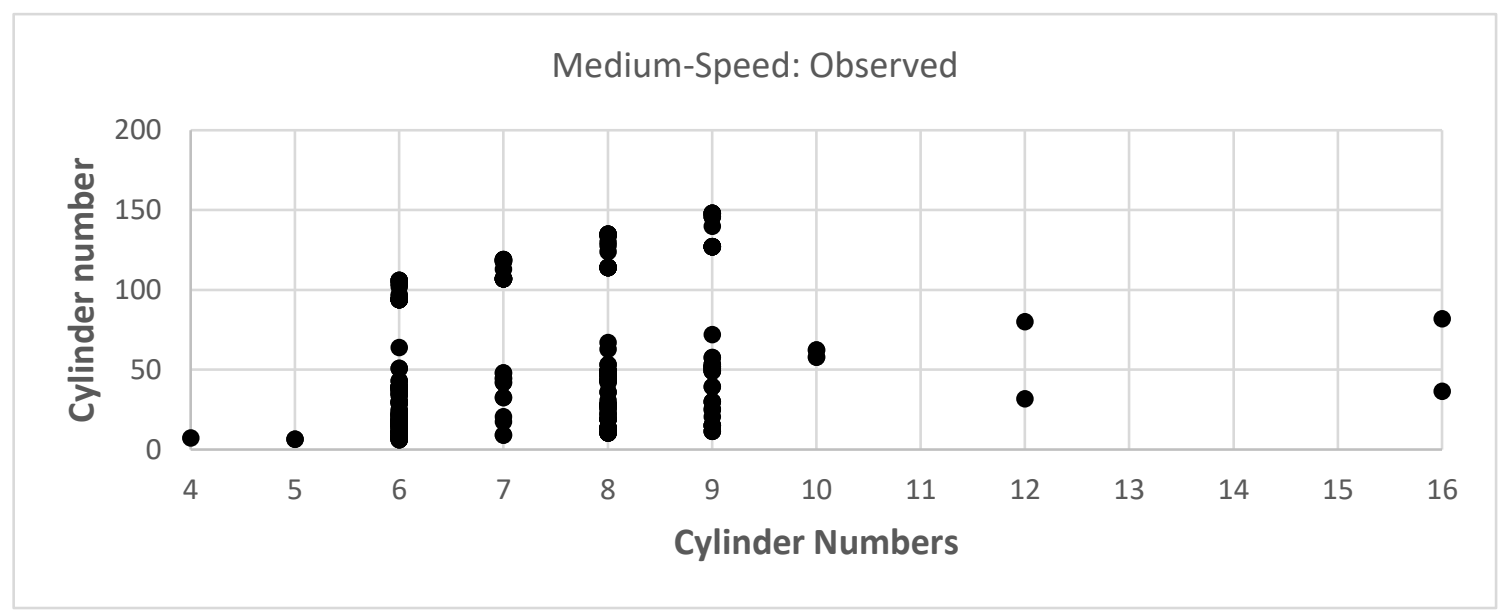

Fig.38 ME weight versus cylinder number of I-type ME (medium-speed)

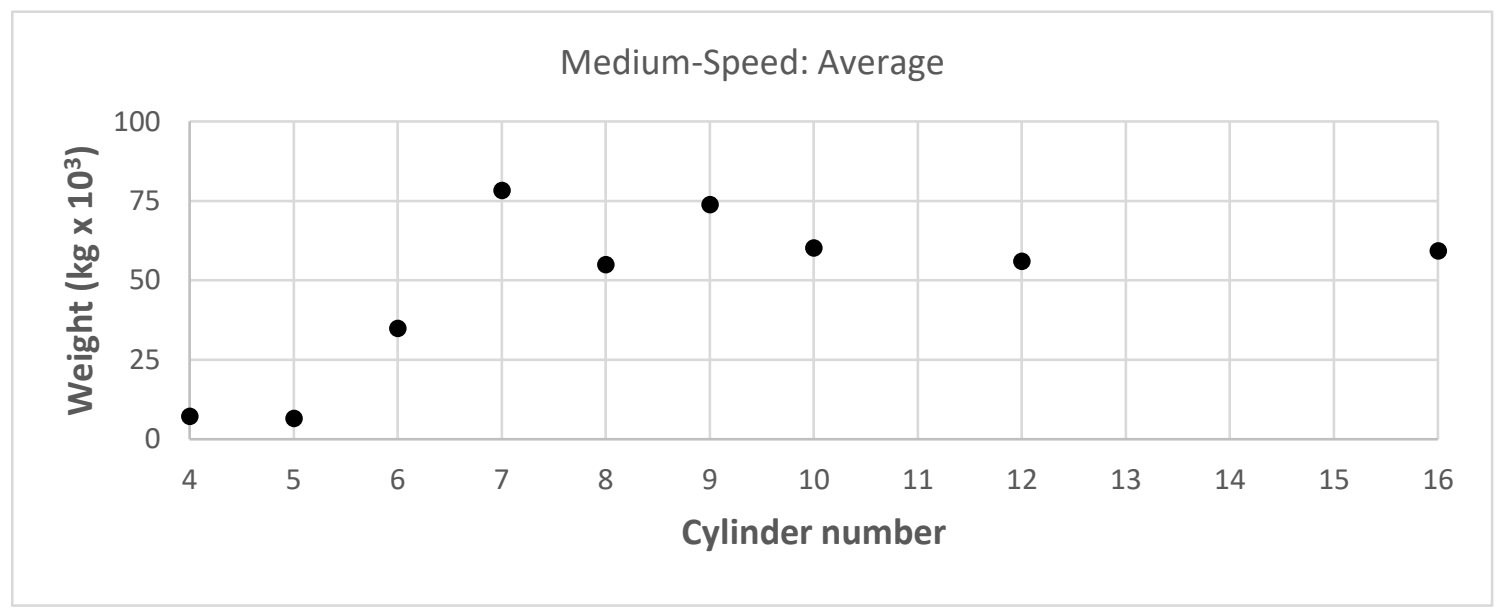

Fig.39 Average ME weight versus cylinder number of I-type ME (medium-speed) 


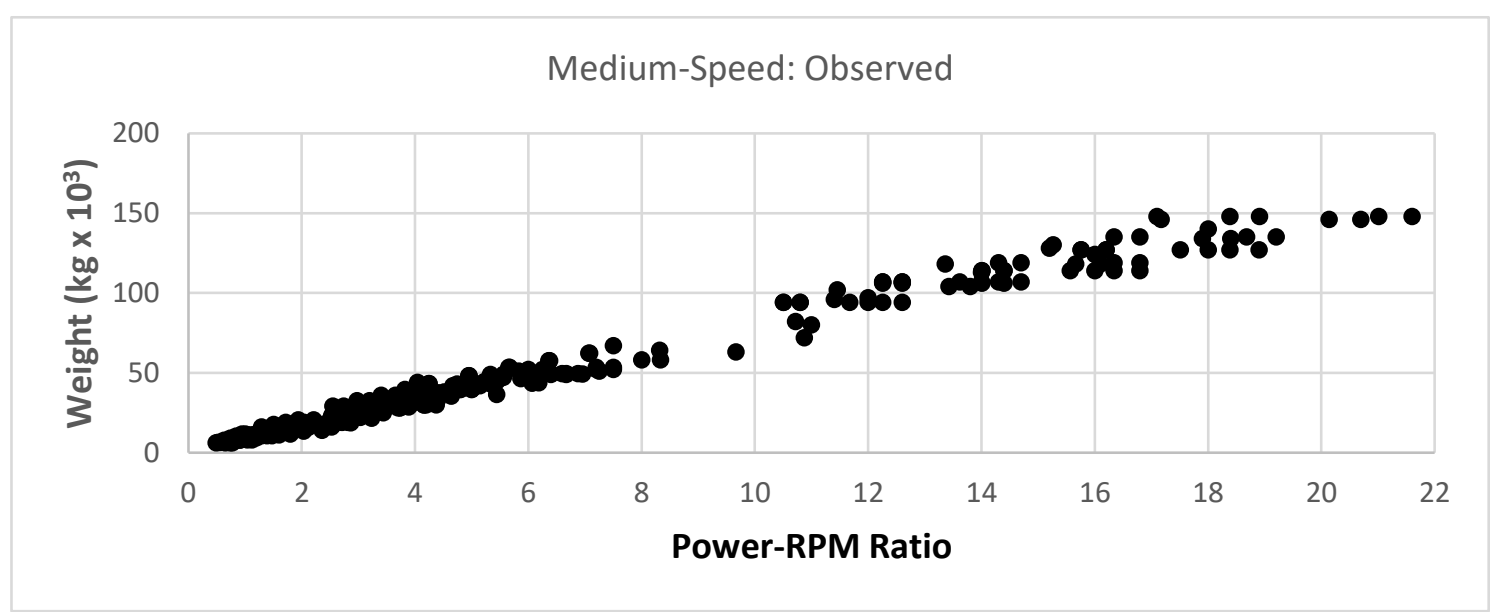

Fig.40 ME weight versus power-speed ratio of I-type ME (medium-speed)

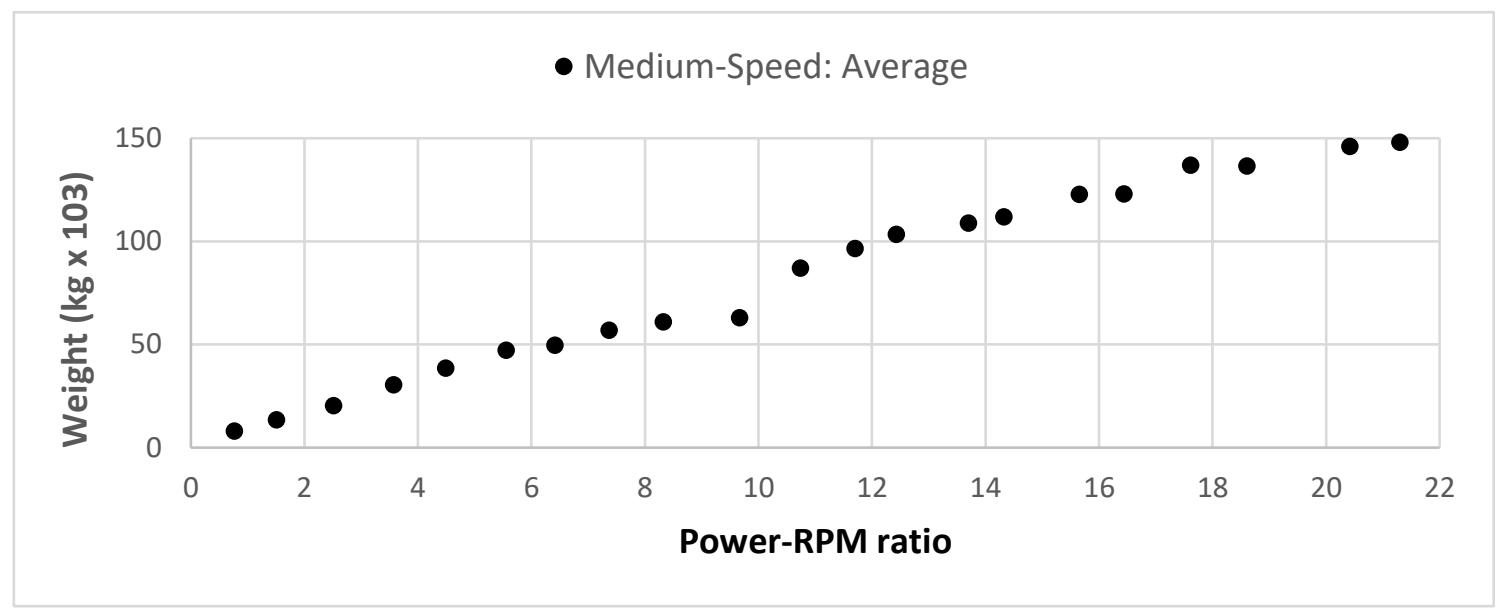

Fig.41 Average ME weight versus average power-RPM ratio of I-type ME (medium-speed)

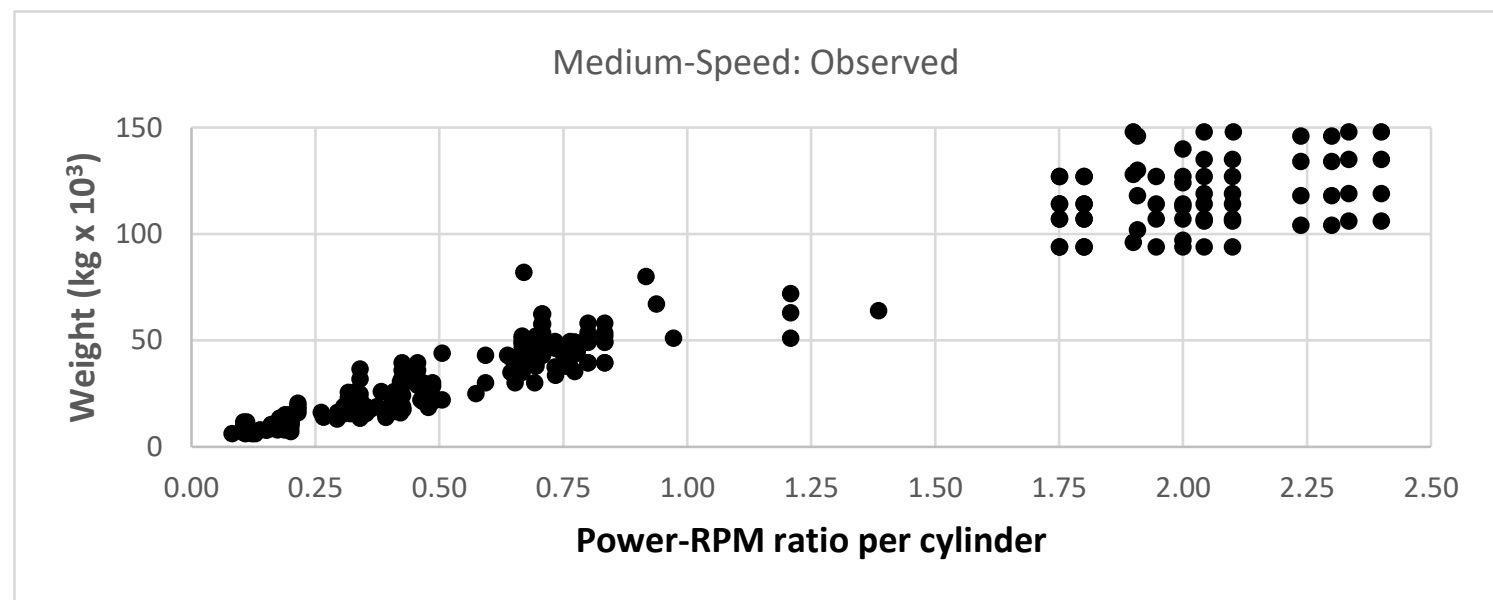

Fig.42 ME weight versus power-RPM ratio per cylinder of I-type ME (medium-speed) 


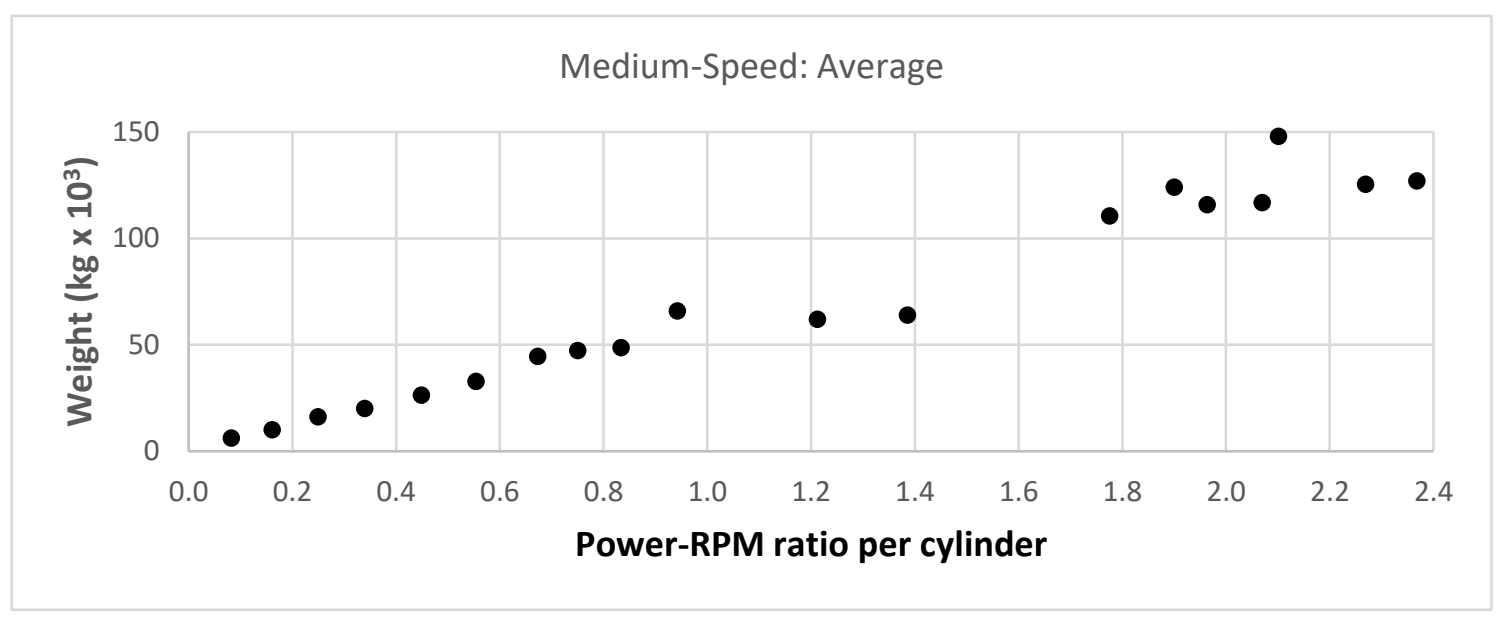

Fig.43 Average ME weight versus average power-RPM ratio per cylinder of I-type ME (medium-speed)

Table 12 Summary of correlation coefficients (for medium-speed engines)

\begin{tabular}{|c|c|c|c|c|c|c|c|}
\hline \multirow{2}{*}{$\begin{array}{c}\text { Figure } \\
\text { No. }\end{array}$} & \multirow{2}{*}{ Variables } & $y=m^{*} x+c$ & $y=m^{*} x$ & $y=m^{*} x^{n}$ & $y=m^{*} e^{b x}$ & $y=a * x^{2}+b * x+c$ & $y=a^{*} \ln (x)+c$ \\
\hline & & \multicolumn{6}{|c|}{ Correlation coefficients $\left(r^{2}\right)$} \\
\hline 34 & \multirow{2}{*}{$W$ vs $P$} & 0.9514 & 0.9400 & 0.9545 & 0.8834 & 0.9528 & 0.7984 \\
\hline 35 & & 0.9802 & 0.9748 & 0.9866 & 0.8941 & 0.9802 & 0.8201 \\
\hline 36 & \multirow{2}{*}{$W$ vs $R P M$} & $0.6096(-)$ & -0.4710 & 0.6263 & 0.6443 & 0.6433 & 0.6267 \\
\hline 37 & & $0.4491(-)$ & -0.4030 & 0.4563 & 0.5166 & 0.4685 & 0.4106 \\
\hline 38 & \multirow{2}{*}{$W$ vs $N$} & 0.0744 & 0.0740 & 0.1313 & 0.1089 & 0.1084 & 0.0915 \\
\hline 39 & & 0.3362 & 0.2958 & 0.5730 & 0.3990 & 0.6962 & 0.4963 \\
\hline 40 & \multirow{2}{*}{$W$ vs $T$} & 0.9822 & 0.9799 & 0.9800 & 0.8426 & 0.9845 & 0.8504 \\
\hline 41 & & 0.9862 & 0.9834 & 0.9937 & 0.8496 & 0.9898 & 0.8577 \\
\hline 42 & \multirow{2}{*}{$W$ vs $T / N$} & 0.9381 & 0.9368 & 0.9454 & 0.8238 & 0.9409 & 0.8504 \\
\hline 43 & & 0.9605 & 0.9602 & 0.9870 & 0.8595 & 0.9605 & 0.8219 \\
\hline
\end{tabular}

\subsubsection{High-speed group}

Main engine weights under low-speed group (speed > 1000 RPM) are analyzed against the power output, RPM, cylinder number, power-speed ratio and power-speed ratio per cylinder to determine the ME weight's behaviour from different viewpoints and presented in Figs.44-53. Their trends are almost identical to that of Figs.14-23 but with different responses and correlation coefficients. Figures are also investigated for other types of relationships, and the results are presented in Table 13. 
High-Speed: Observed

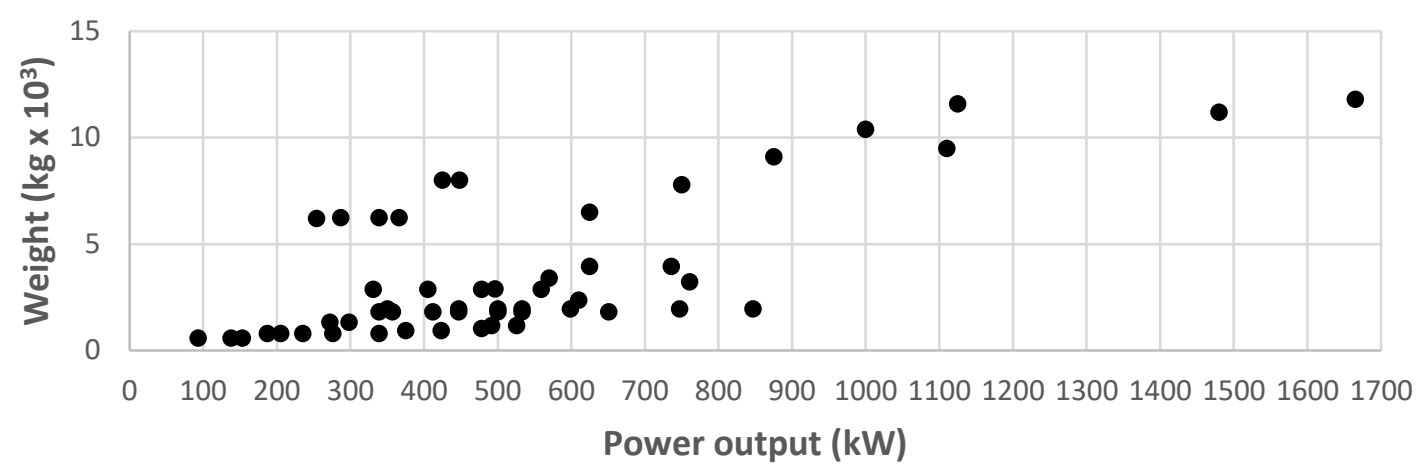

Fig.44 ME weight versus power output of I-type ME (high-speed)

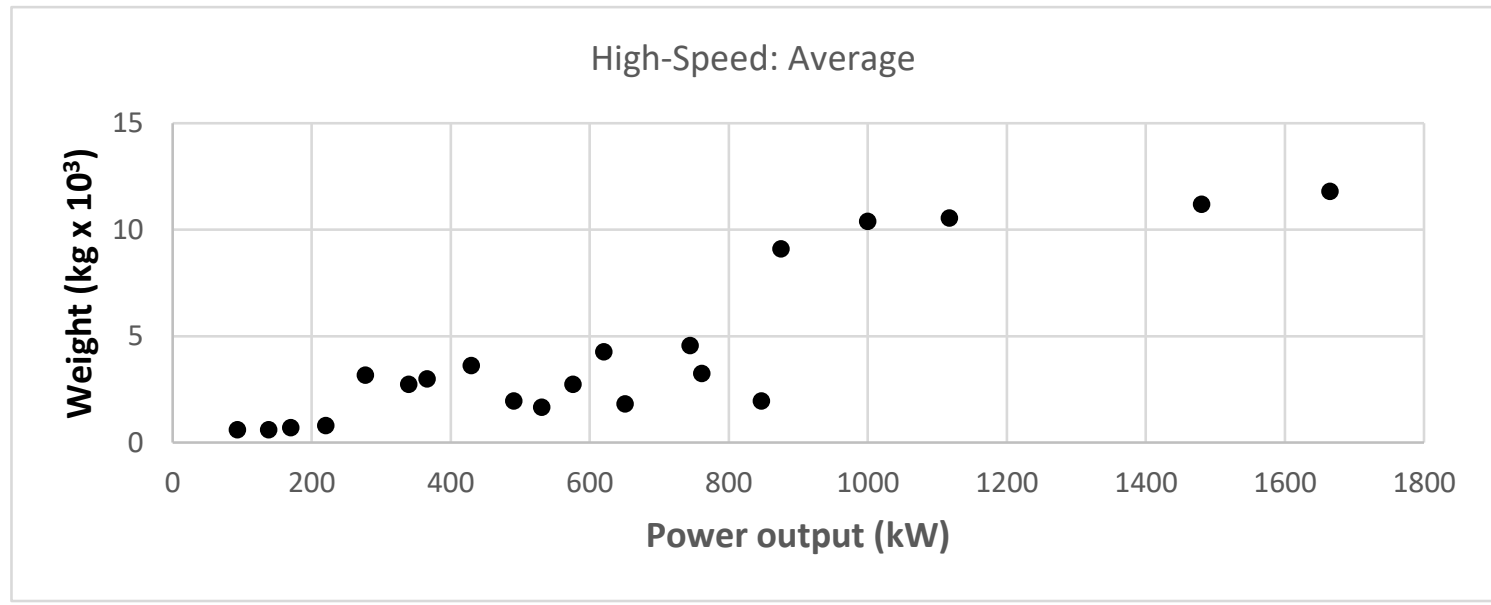

Fig.45 Average ME weight versus average power output of I-type ME (high-speed)

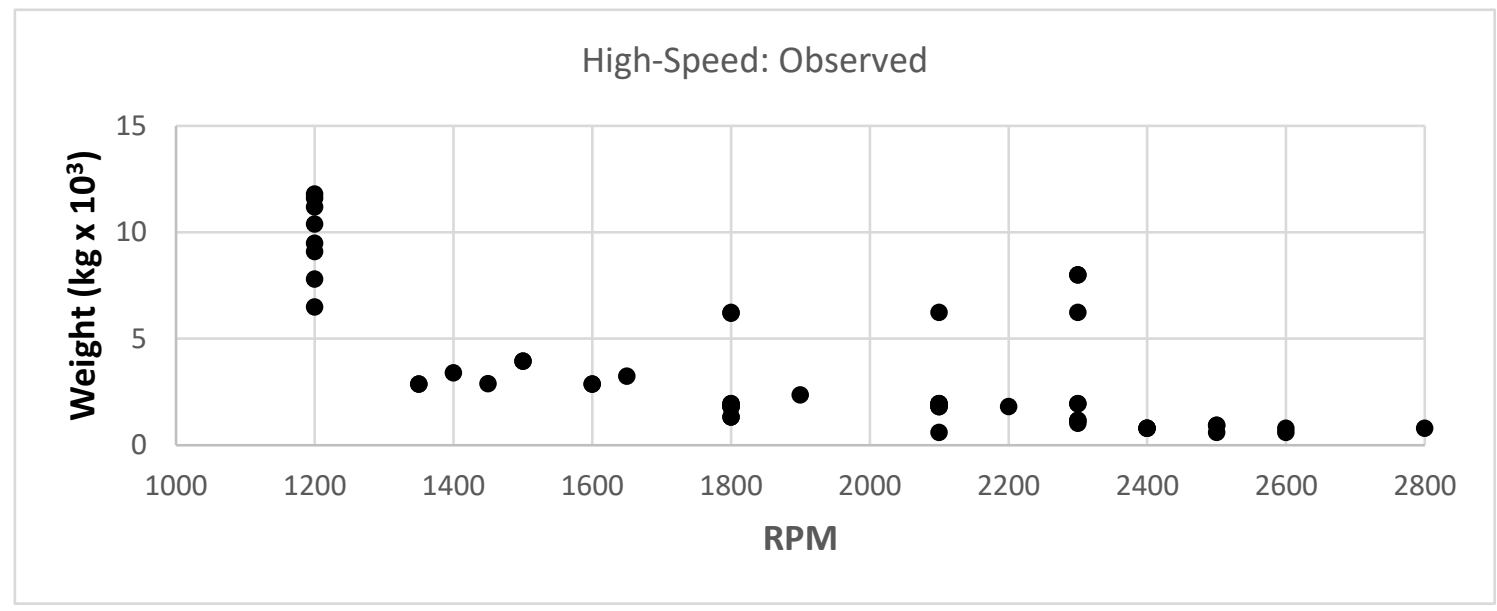

Fig.46 ME weight versus RPM of I-type ME (high-speed) 


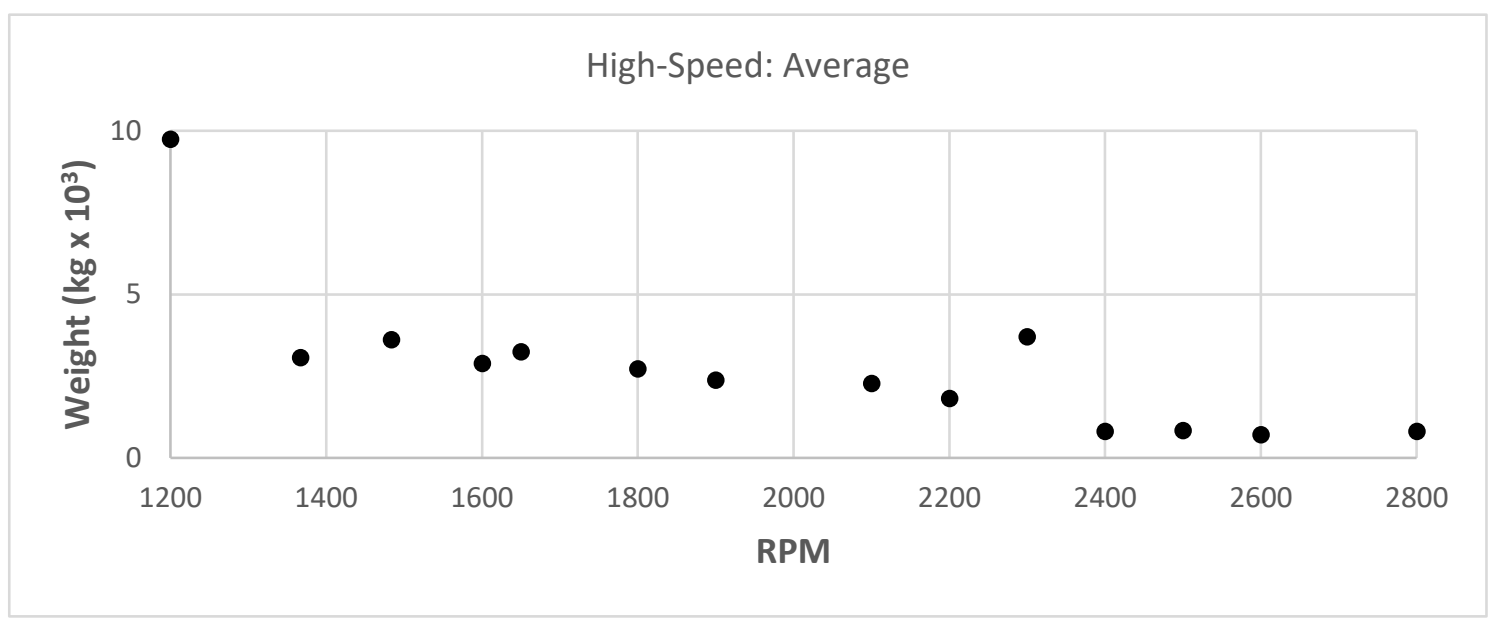

Fig.47 Average ME weight versus average RPM of I-type ME (high-speed)

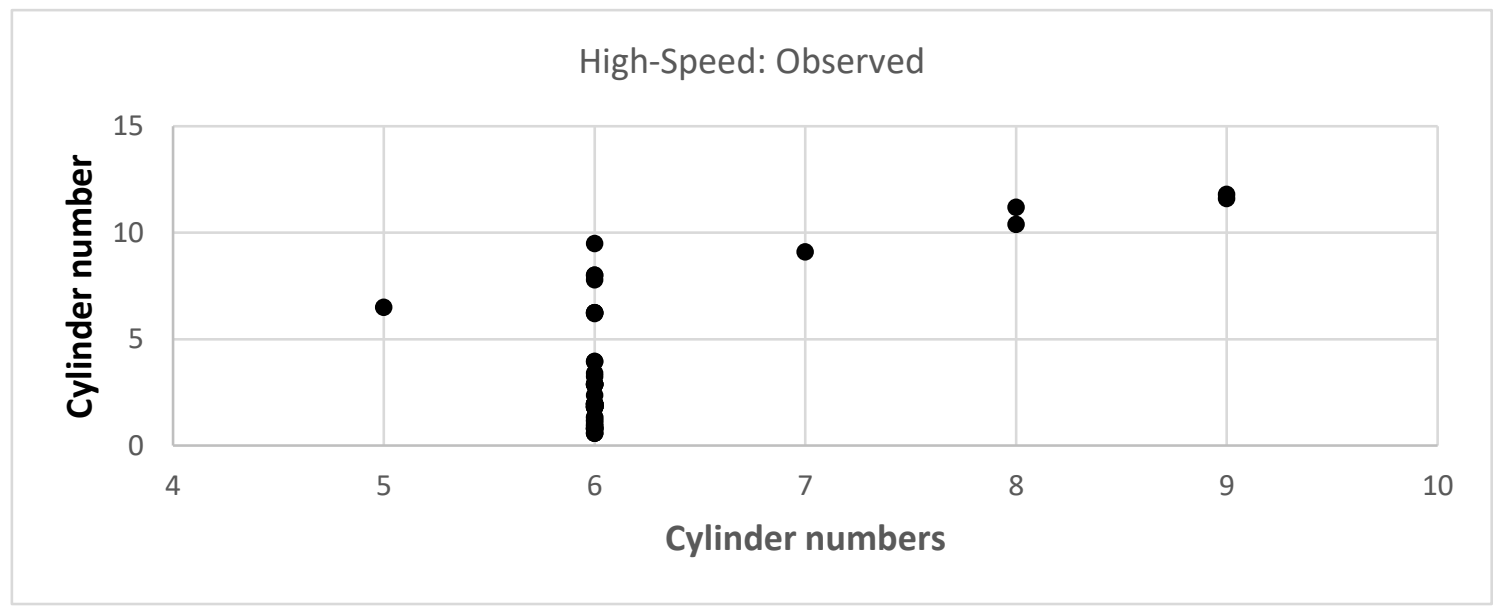

Fig.48 ME weight versus cylinder number of I-type ME (high-speed)

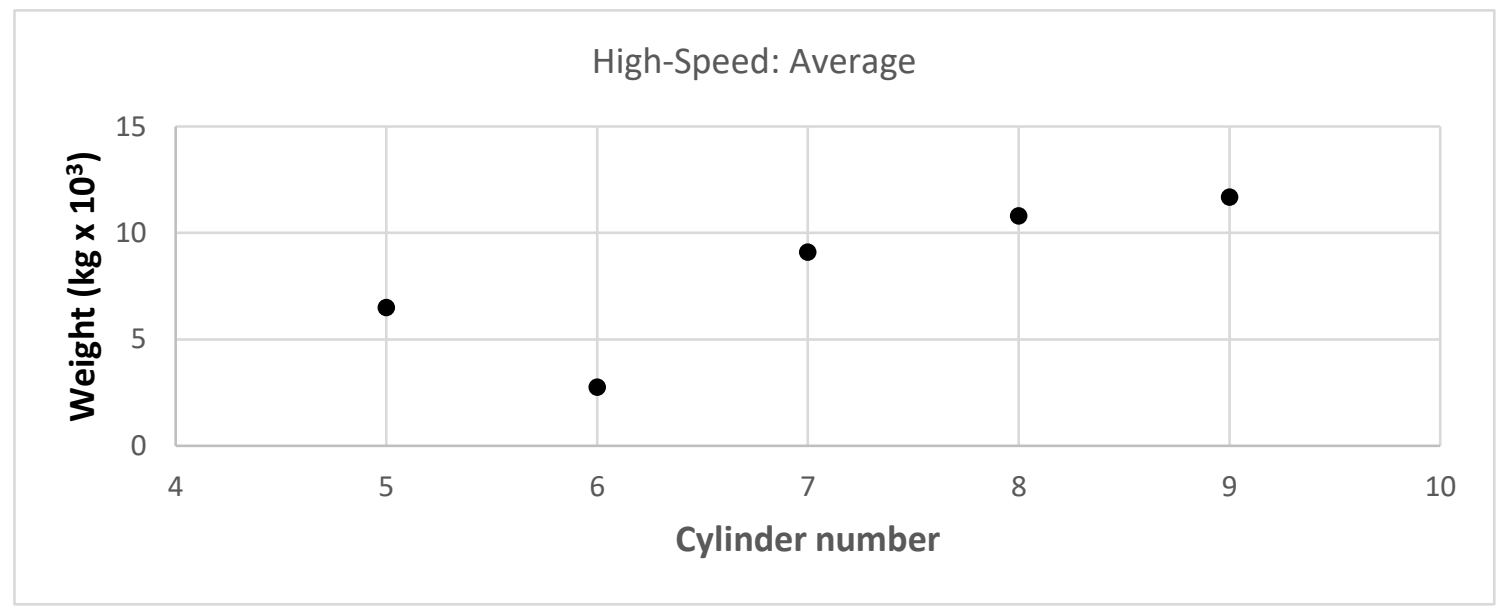

Fig.49 Average ME weight versus cylinder number of I-type ME (high-speed) 
High-Speed: Observed

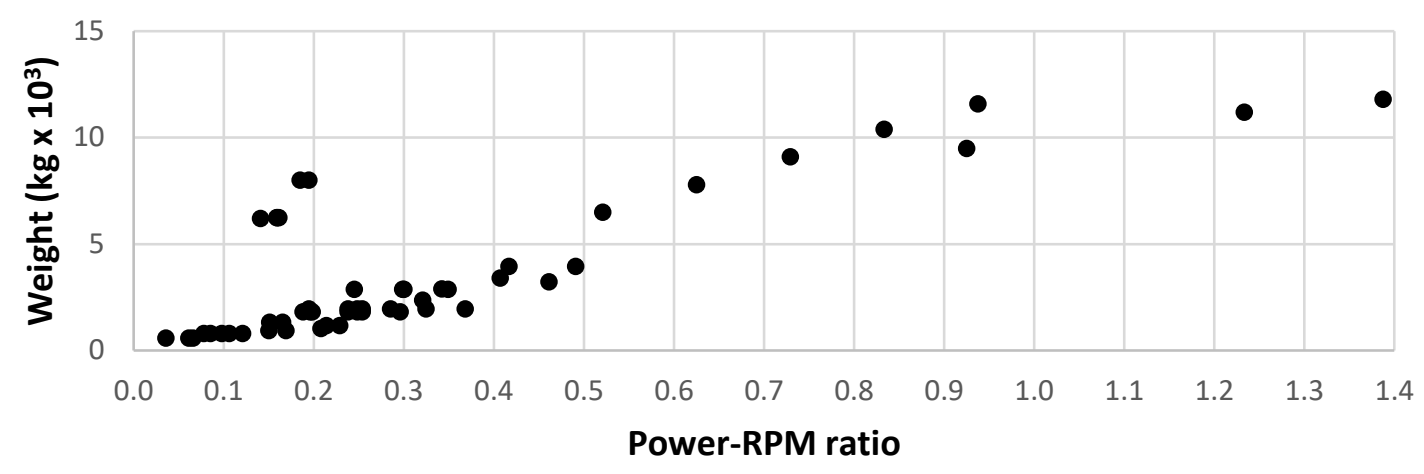

Fig.50 ME weight versus power-RPM ratio of I-type ME (high-speed)

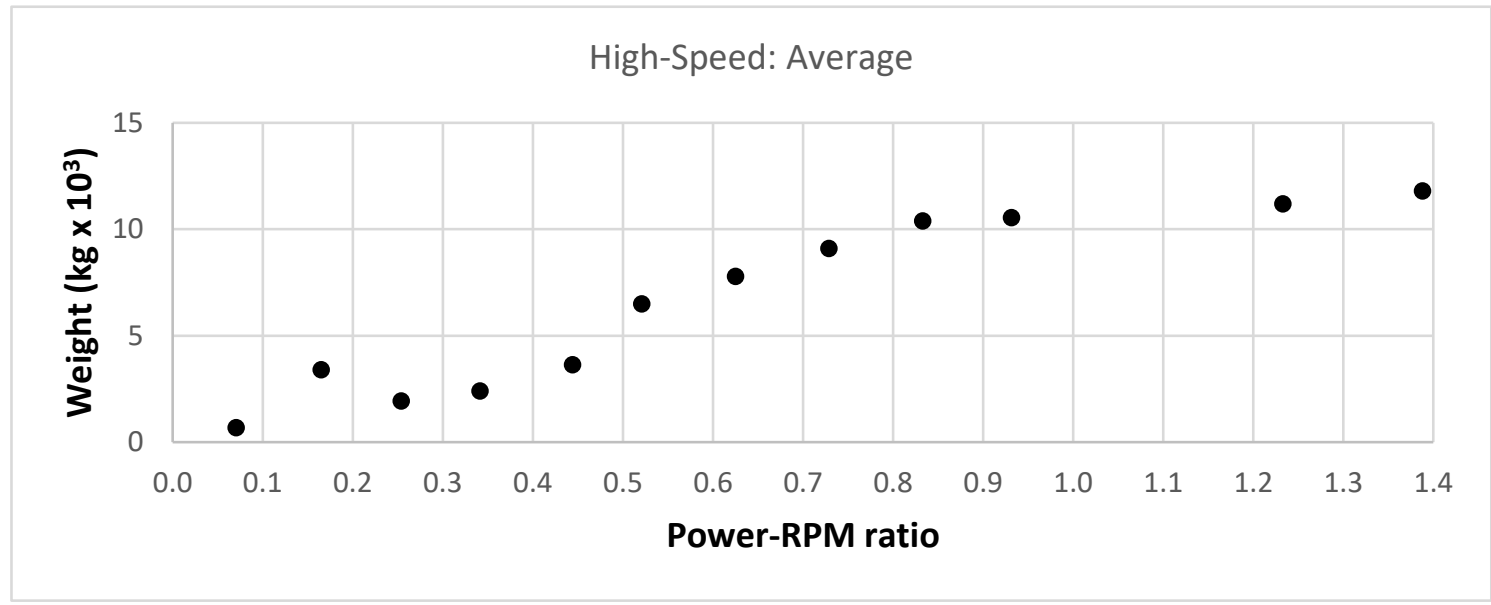

Fig.51 Average ME weight versus average power-RPM ratio of I-type ME (high-speed)

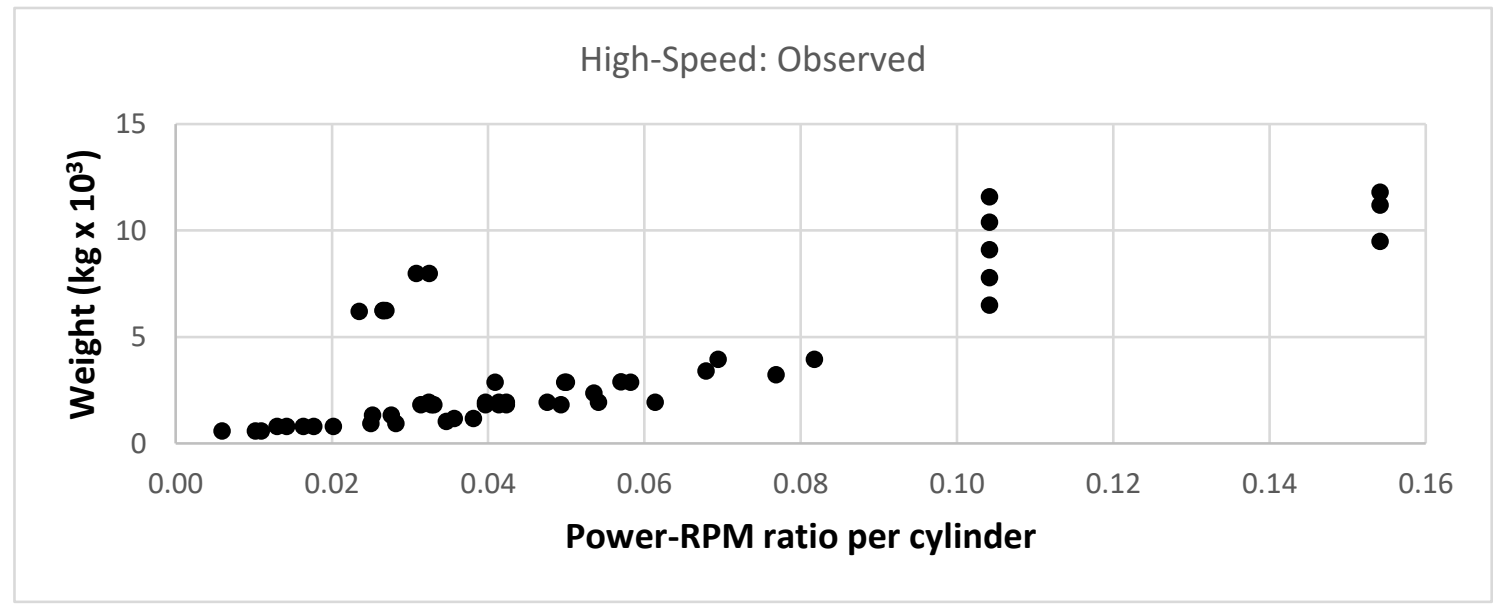

Fig.52 ME weight versus power-RPM ratio per cylinder of I-type ME (high-speed) 


\section{High-Speed: Average}

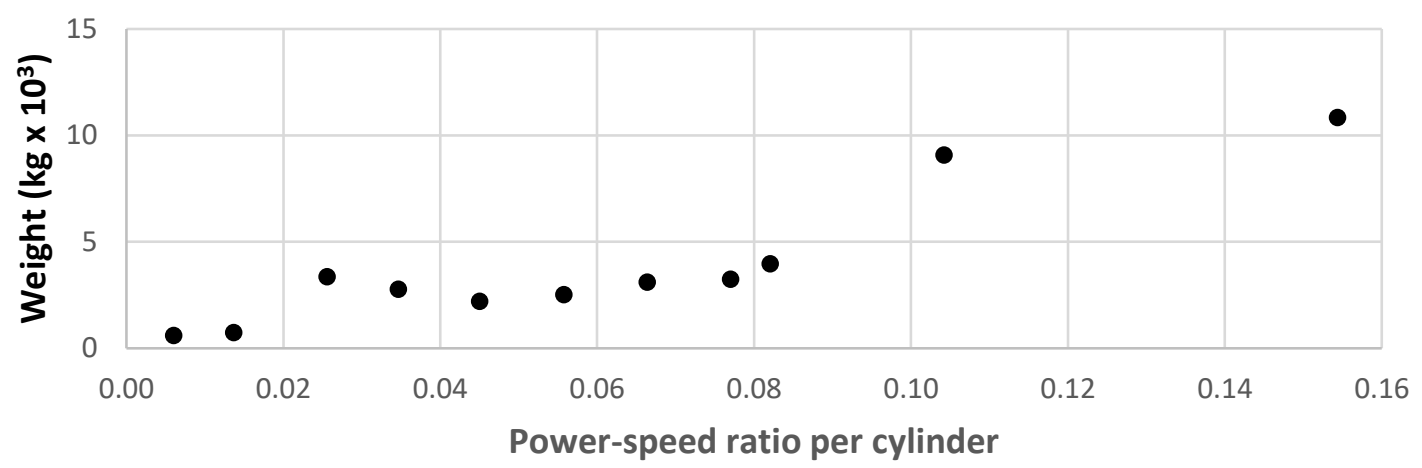

Fig.53 Average weight versus average power-RPM ratio per cylinder of I-type ME (high-speed)

Table 13 Summary of correlation coefficients (for high-speed engines)

\begin{tabular}{|c|c|c|c|c|c|c|c|}
\hline \multirow{2}{*}{$\begin{array}{c}\text { Figure } \\
\text { No. }\end{array}$} & \multirow{2}{*}{ Variables } & $y=m^{*} x+c$ & $y=m * x$ & $y=m^{*} x^{n}$ & $y=m^{*} e^{b x}$ & $y=a * x^{2}+b * x+c$ & $y=a * \ln (x)+c$ \\
\hline & & \multicolumn{6}{|c|}{ Correlation coefficients $\left(r^{2}\right)$} \\
\hline 44 & \multirow{2}{*}{$W$ vs $P$} & 0.5251 & 0.5208 & 0.4707 & 0.4526 & 0.5361 & 0.3920 \\
\hline 45 & & 0.7780 & 0.7657 & 0.7669 & 0.7068 & 0.7815 & 0.6057 \\
\hline 46 & \multirow{2}{*}{$W$ vs $R P M$} & $0.4219(-)$ & -0.3710 & 0.5470 & 0.5352 & 0.5197 & 0.4707 \\
\hline 47 & & $0.5503(-)$ & -0.4760 & 0.7426 & 0.7507 & 0.6510 & 0.6088 \\
\hline 48 & \multirow{2}{*}{$W$ vs $N$} & 0.4485 & 0.1573 & 0.2216 & 0.2332 & 0.4646 & 0.4310 \\
\hline 49 & & 0.6501 & 0.5660 & 0.4333 & 0.4660 & 0.6795 & 0.6150 \\
\hline 50 & \multirow{2}{*}{$W$ vs $T$} & 0.6452 & 0.6306 & 0.5924 & 0.5185 & 0.6452 & 0.4986 \\
\hline 51 & & 0.8819 & 0.8677 & 0.8894 & 0.7288 & 0.9284 & 0.8324 \\
\hline 52 & \multirow{2}{*}{$W$ vs $T / N$} & 0.5893 & 0.5888 & 0.5716 & 0.5442 & 0.6604 & 0.4379 \\
\hline 53 & & 0.8334 & 0.8316 & 0.8387 & 0.7740 & 0.8666 & 0.5735 \\
\hline
\end{tabular}

Table 14 is the summary of Tables 11-13. It shows the improvement in $r^{2}$ values under three speed groups except for high-speed group.

Table 14 Comparison of correlation coefficients

\begin{tabular}{|c|c|c|c|c|c|}
\hline & & & \multicolumn{3}{|c|}{ Speed groups } \\
\hline \multirow{2}{*}{ Variables } & \multirow{2}{*}{ Equation form } & Combined & Low & Medium & High \\
\hline & & \multicolumn{4}{|c|}{ Correlation coefficients $\left(r^{2}\right)$} \\
\hline \multirow{3}{*}{$W=f(P)$} & $\mathrm{W}=\mathrm{a}+\mathrm{b} * P$ & 0.9544 & 0.9531 & 0.9802 & 0.7780 \\
\hline & $\mathrm{W}=\mathrm{a} * p^{\mathrm{n}}$ & 0.9798 & 0.9859 & 0.9866 & 0.7669 \\
\hline & $\mathrm{W}=\mathrm{a} * P^{2}+\mathrm{b} * P+\mathrm{c}$ & 0.9743 & 0.9730 & 0.9802 & 0.7815 \\
\hline \multirow{3}{*}{$W=f(T)$} & $\mathrm{W}=\mathrm{a}+\mathrm{b}^{*} T$ & 0.9774 & 0.9777 & 0.9862 & 0.8819 \\
\hline & $\mathrm{W}=\mathrm{a} * T^{\mathrm{n}}$ & 0.9955 & 0.9952 & 0.9937 & 0.8894 \\
\hline & $\mathrm{W}=\mathrm{a} * T^{2}+\mathrm{b} * T+\mathrm{c}$ & 0.9895 & 0.9896 & 0.9898 & 0.9284 \\
\hline \multirow{2}{*}{$W=f(T / N)$} & $\mathrm{W}=\mathrm{a}+\mathrm{b}^{*}(T / N)$ & 0.9775 & 0.9822 & 0.9605 & 0.8334 \\
\hline & $\mathrm{W}=\mathrm{a} *(T / N)^{\mathrm{n}}$ & 0.9838 & 0.9879 & 0.9870 & 0.8387 \\
\hline
\end{tabular}




$$
\begin{array}{|l|l|l|l|l|}
\mathrm{W}=\mathrm{a} *(T / N)^{2}+\mathrm{b} *(T / N)+\mathrm{c} & 0.9783 & 0.9822 & 0.9605 & 0.8666 \\
\hline
\end{array}
$$

\subsection{Power generation machinery weight analysis}

Initial investigations on pairs of variables of interests related to AE weight are shown in Figs.54-63. Pairs of variables refer to a combination of the dependent variable, the AE weight and an independent variable, such as generator power output, cylinder number, RPM, power-RPM ratio, and power-RPM ratio per cylinder. Correlation coefficients are calculated for each relationship under different equation forms for comparison purpose and presented in tabular form in Tables 15-19.

\subsubsection{Generator power output}

Auxiliary engine weights are analyzed against the power output to determine the AE weight's behaviour from different viewpoints and presented in Figs.54-55. Figure 54 shows the AE weight (observed values) trend against the power output, and it seems to be linearly associated. Figure 55 shows the behaviour of average AE weight (average by power output group) against average power output. It also shows the sign of linearity among variables. This shows that with the rise in power output, the AE weight increases linearly. Therefore, it is expected that with the increase of power output, the AE weight would be increased too. This is in line with the second assumption.

Figures are additionally investigated for other forms of relationships, and the result is presented in Table 15. Table 15 shows the correlation coefficients under different equation forms, and it suggests that power form and polynomial form (second-order) yield high correlation coefficients for Figs.54 and 55 and that those are not very far apart.

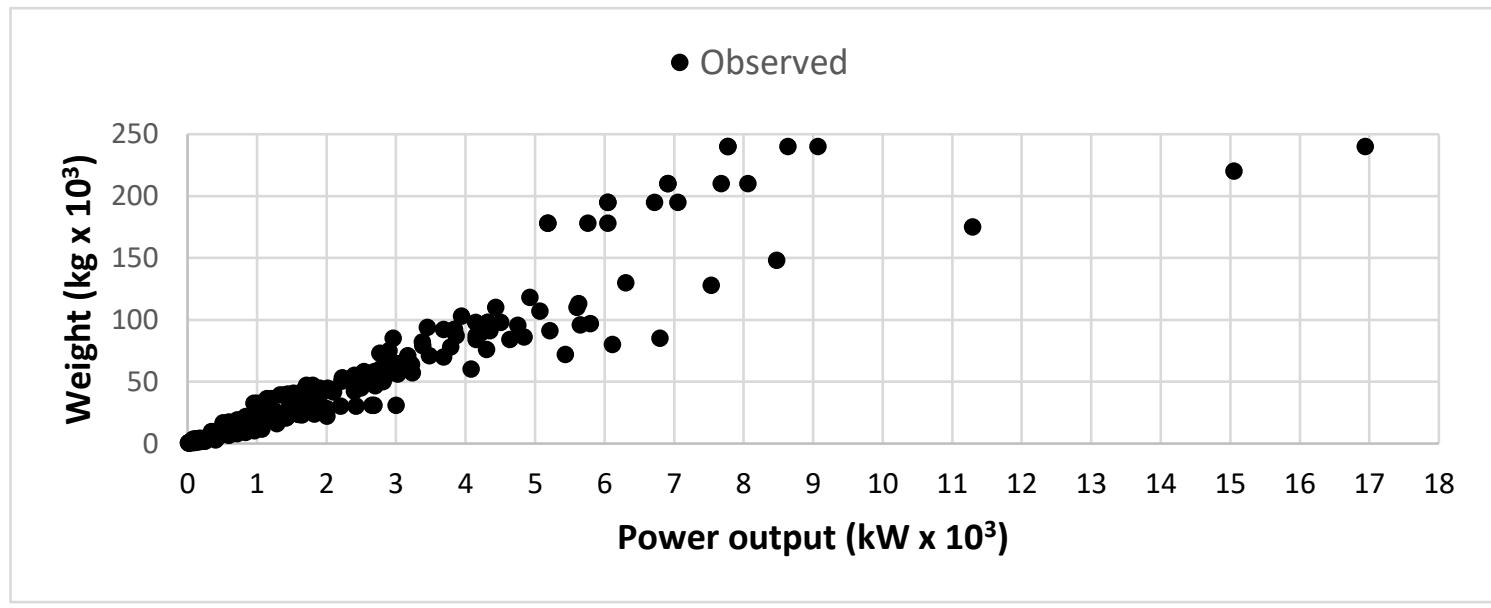

Fig.54 AE weight versus power output of I-type $A E$ 


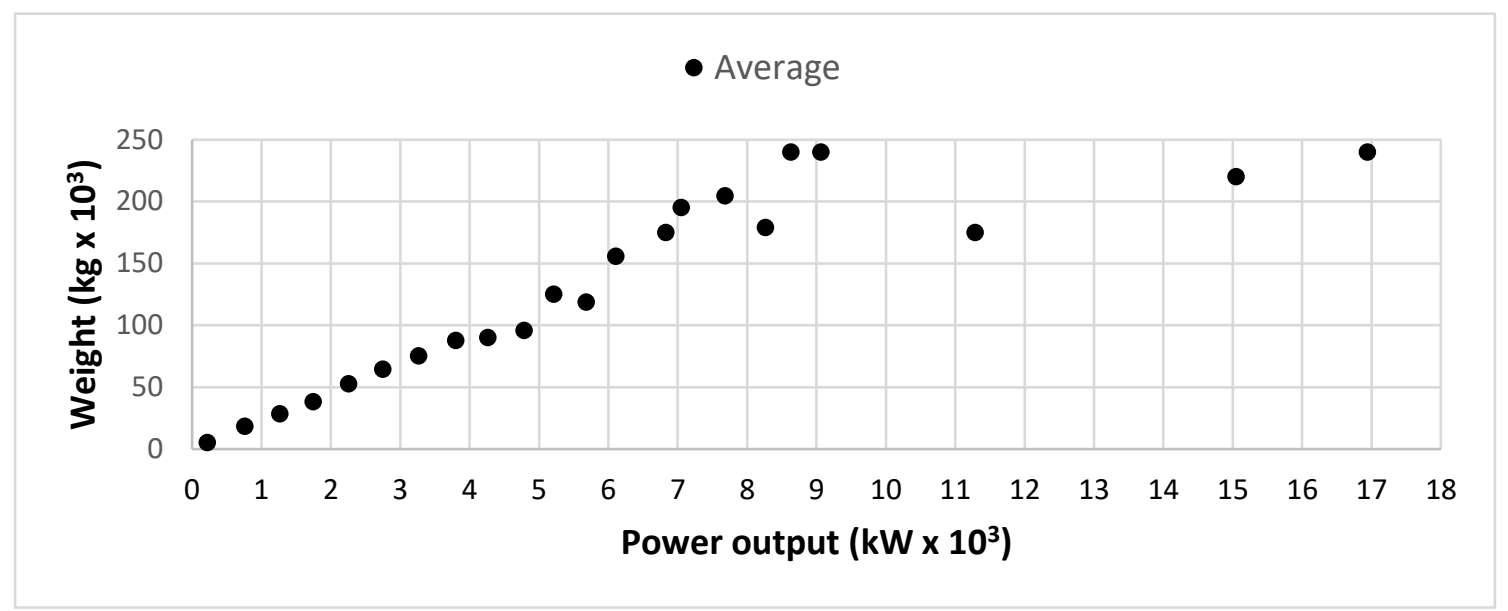

Fig.55 Average AE weight versus average power output of I-type AE

Table 15 Summary of correlation coefficients

\begin{tabular}{|c|c|c|c|c|c|c|c|}
\hline \multirow{2}{*}{$\begin{array}{c}\text { Figure } \\
\text { No. }\end{array}$} & Variables & $y=m^{*} x+c$ & $y=m^{*} x$ & $y=m^{*} x^{n}$ & $y=m^{*} e^{b x}$ & $y=a^{*} x^{2}+b^{*} x+c$ & $y=a * \ln (x)+c$ \\
\cline { 3 - 8 } & & \multicolumn{7}{|c|}{ Values of correlation coefficients $\left(r^{2}\right)$} & 0.887 & 0.5568 \\
\hline 54 & $W$ vs $P$ & 0.8565 & 0.8563 & 0.9562 & 0.5974 & 0.9200 & 0.7842 \\
\hline 55 & $W$ vs $P$ & 0.7818 & 0.7171 & 0.9662 & 0.6054 & 0.962 \\
\hline
\end{tabular}

\subsubsection{Engine RPM}

Auxiliary engine weights are analyzed against the RPM to determine the behaviour of the AE weight from different viewpoints, and the results are presented in Figs.56-57. Figure 56 illustrates the trend of the AE weight (observed values) against RPM. Figure 57 demonstrates the behaviour of average AE weight (average by RPM group) against average RPM. It also shows the sign of power form of relationship among variables. This shows that with the rise in RPM, the AE weight decreases. Therefore, it is expected that with the increase in RPM, the AE weight would be reduced. This is in line with the third assumption, but in the power form, instead of a linear form of an equation.

Figures are additionally investigated for other forms of relationships, and the results are presented in Table 16. Table 16 shows the correlation coefficients under different equation forms, and it suggests that power form yields the highest correlation coefficient for Figs. 56 and 57, respectively. 


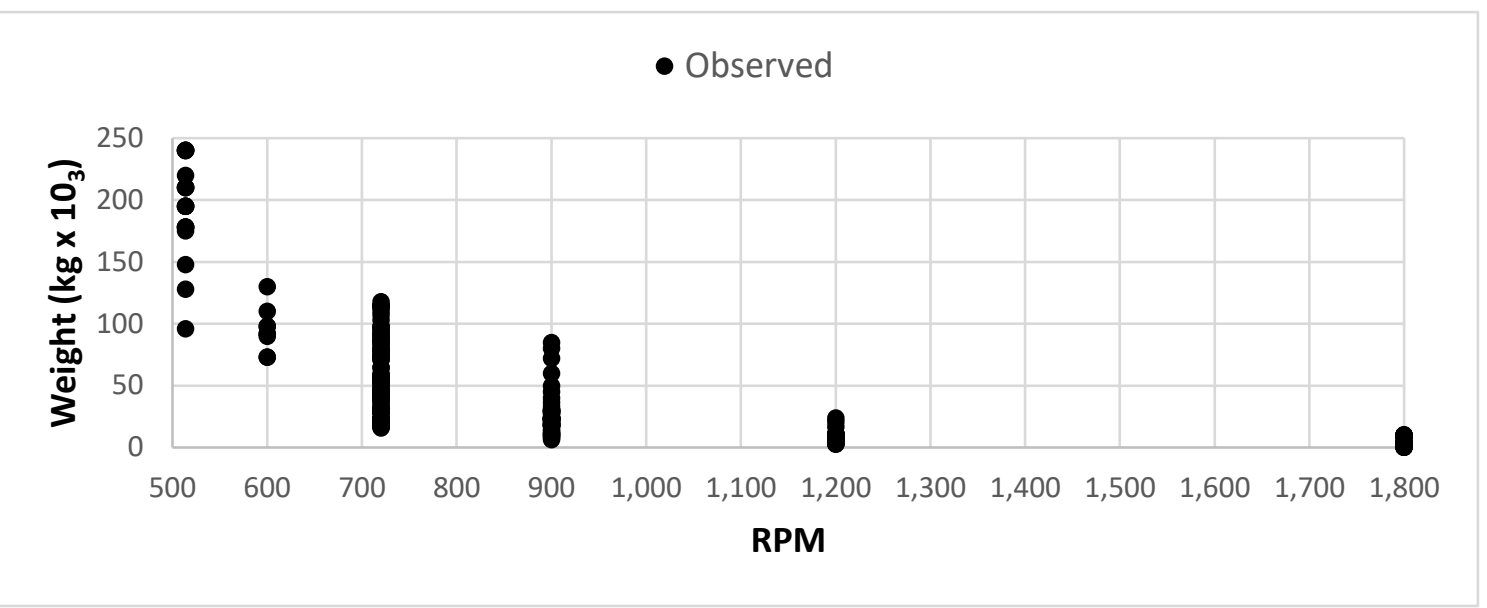

Fig.56 AE weight versus RPM of I-type AE

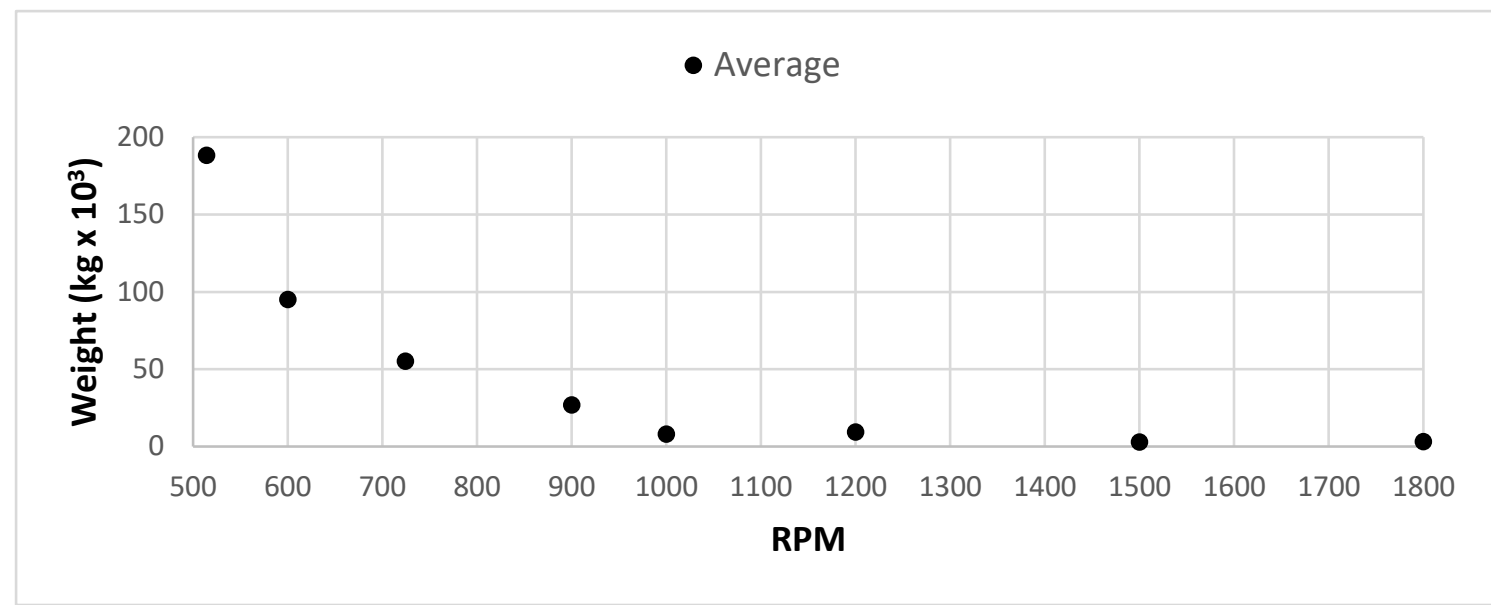

Fig.57 Average AE weight versus average RPM of I-type AE

Table 16 Summary of correlation coefficients

\begin{tabular}{|c|c|c|c|c|c|c|c|}
\hline \multirow{2}{*}{$\begin{array}{c}\text { Figure } \\
\text { No. }\end{array}$} & \multirow{2}{*}{ Variables } & $y=\mathrm{m}^{*} x+\mathrm{c}$ & $y=\mathrm{m}^{*} x$ & $y=\mathrm{m}^{*} x^{\mathrm{n}}$ & $y=\mathrm{m}^{*} \mathrm{e}^{\mathrm{b} x}$ & $y=\mathrm{a}^{*} x^{2}+\mathrm{b}^{*} x+\mathrm{c}$ & $y=\mathrm{a} * \ln (x)+\mathrm{c}$ \\
\cline { 3 - 8 } & & \multicolumn{7}{|c|}{ Values of correlation coefficients $\left(r^{2}\right)$} \\
\hline 56 & $W$ vs RPM & $0.3835(-)$ & -0.4540 & 0.8260 & 0.7945 & 0.6957 & 0.5042 \\
\hline 57 & $W$ vs RPM & $0.5331(-)$ & -0.4300 & 0.9510 & 0.8794 & 0.8765 & 0.7213 \\
\hline
\end{tabular}

\subsubsection{Cylinder number}

Auxiliary engine weights are analyzed against the cylinder number to determine the AE weight's behaviour from different viewpoints and presented in Figs.58-59. Figure 58 shows the trend of the $A E$ weight (observed values) against the cylinder number. Figures 59 shows the pattern of average $A E$ weight (average by cylinder number) against cylinder number. It also shows the sign of linearity among variables. Although the observed values offer a full range of the $A E$ weight against the cylinder number, it demonstrates that the $A E$ weight increases linearly with the increase of the engines' 
cylinder number. Therefore, it is expected that with the rise in the cylinder number, the AE weight would be increased too. This is in line with the fourth assumption.

Figures are additionally investigated for other forms of relationships, and the results are presented in Table 17. Table 17 shows the correlation coefficients under different equation forms, and it suggests that power form and polynomial form (second-order) yield the highest correlation coefficients for Figs.58 and 59, respectively.

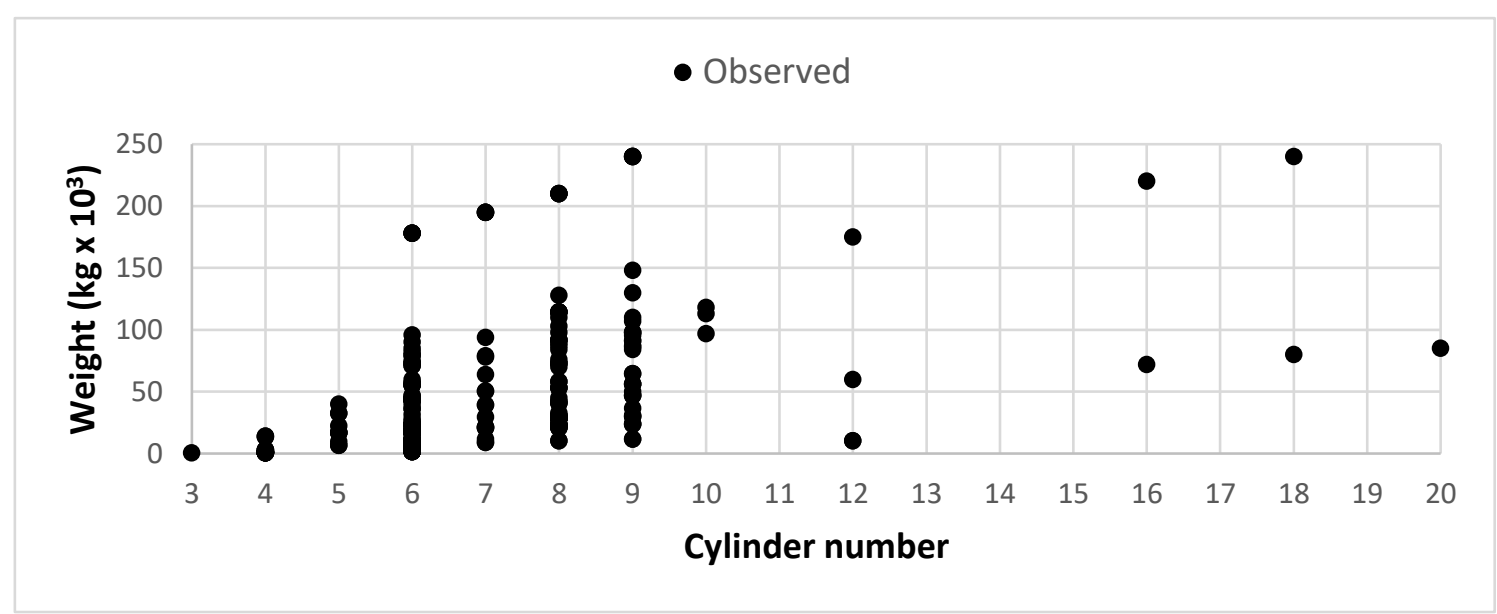

Fig.58 AE weight versus cylinder number of I-type AE

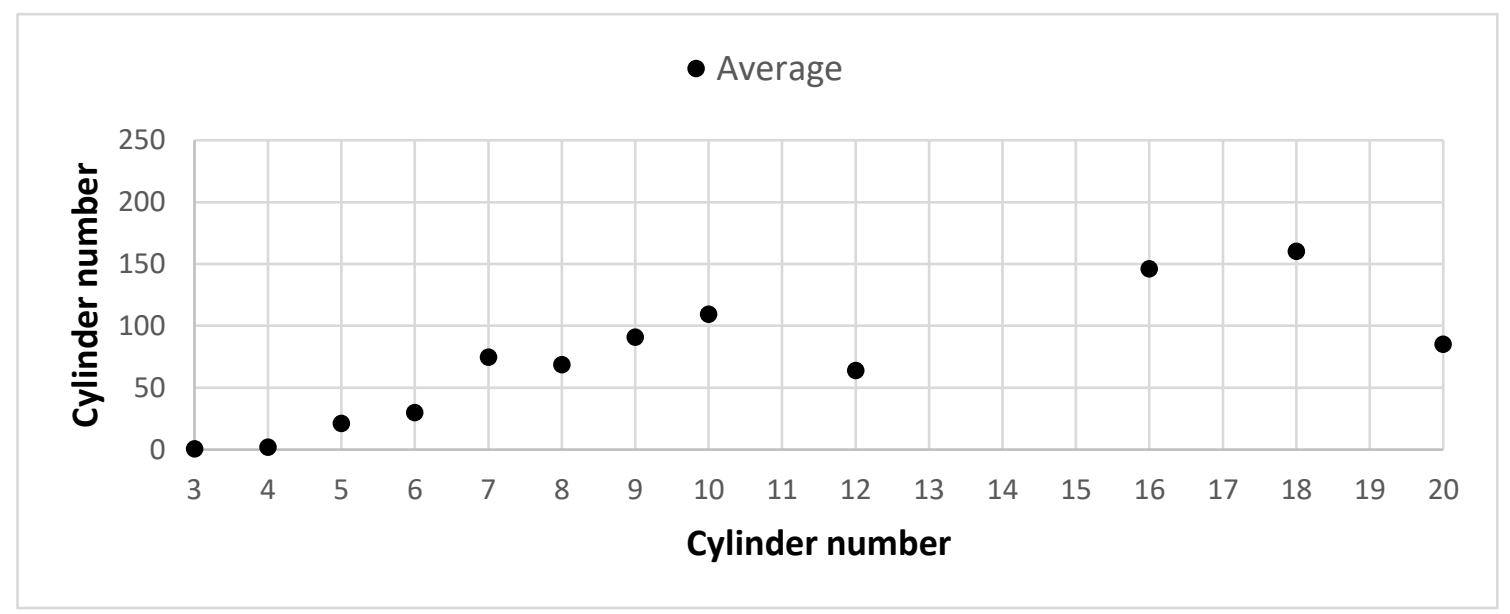

Fig.59 Average AE weight versus cylinder number of I-type AE

Table 17 Summary of correlation coefficients

\begin{tabular}{|c|c|r|r|r|r|r|r|r|}
\hline \multirow{2}{*}{$\begin{array}{c}\text { Figure } \\
\text { No. }\end{array}$} & \multirow{2}{*}{ Variables } & $y=\mathrm{m}^{*} x+\mathrm{c}$ & $y=\mathrm{m}^{*} x$ & $y=\mathrm{m}^{*} x^{\mathrm{n}}$ & $y=\mathrm{m}^{*} \mathrm{e}^{\mathrm{bx}}$ & $y=\mathrm{a}^{*} x^{2}+\mathrm{b}^{*} x+\mathrm{c}$ & $y=\mathrm{a} \ln (x)+\mathrm{c}$ \\
\cline { 3 - 8 } & & \multicolumn{6}{|c|}{ Values of correlation coefficients $\left(r^{2}\right)$} \\
\hline 58 & $W$ vs $N$ & 0.2265 & 0.1867 & 0.4077 & 0.2986 & 0.2554 & 0.2491 \\
\hline 59 & $W$ vs $N$ & 0.6567 & 0.6559 & 0.7114 & 0.4759 & 0.8033 & 0.7559 \\
\hline
\end{tabular}




\subsubsection{Power-RPM ratio}

Auxiliary engine weights are analyzed against the power-RPM ratio to determine the AE weight's behaviour from different viewpoints and presented in Figs.60-61. Figure 60 demonstrates the trend of the AE weight (observed values) against the power-RPM ratio. Figure 61 illustrates the behaviour of average AE weight (average by power-RPM ratio group) against average power-RPM ratio. It also shows the sign of linearity among variables. This shows that with the rise in power-RPM ratio, the AE weight increases linearly. Therefore, it is expected that with the increase of power-RPM ratio, the AE weight would be increased too.

Figures are additionally investigated for other forms of relationships, and the results are presented in Table 18. Table 18 shows the correlation coefficients under different equation forms, and it suggests that the power form yields the highest correlation coefficients for Fig.60 and 61, but yields from polynomial (second-order) type are not very far from the highest values.

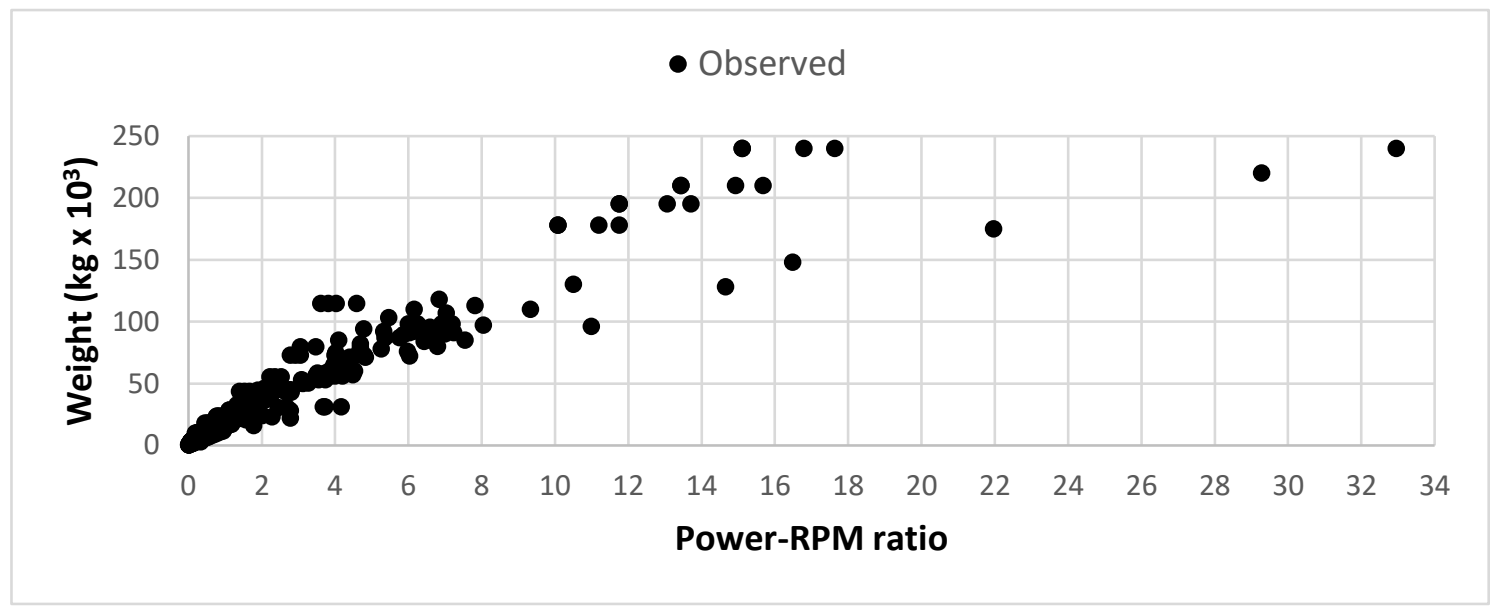

Fig.60 AE weight versus power-RPM ratio of I-type AE

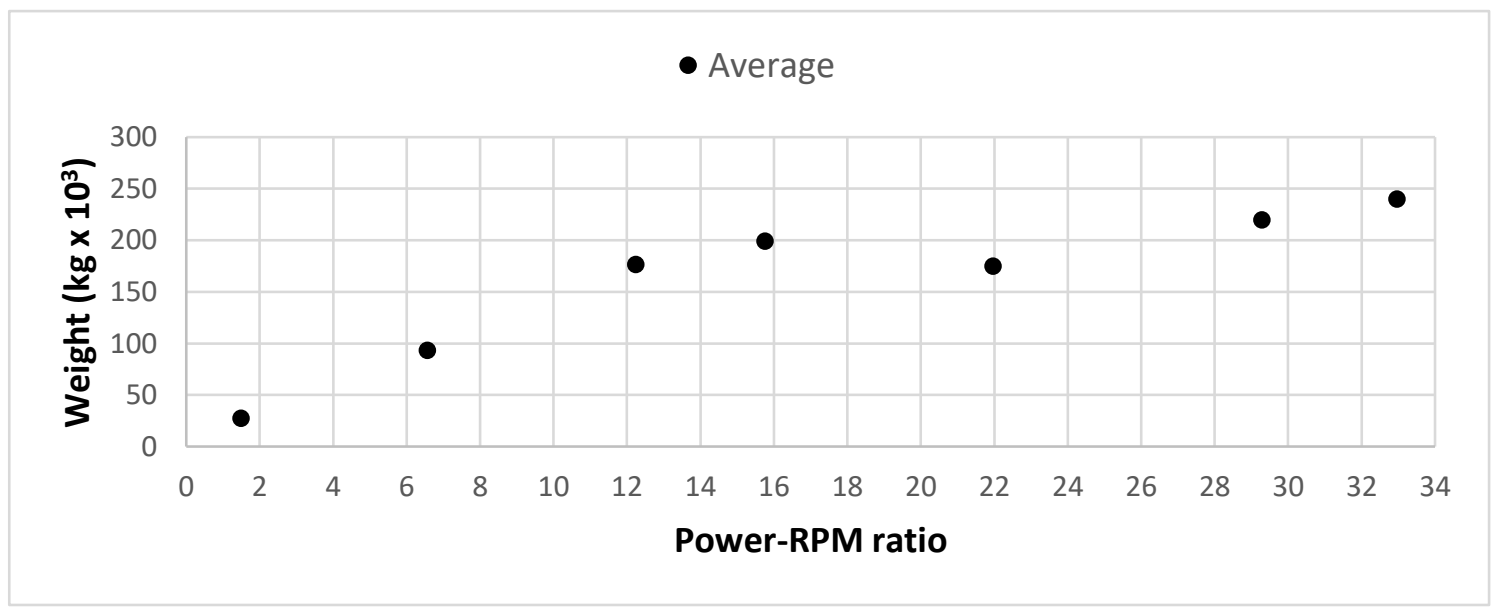

Fig.61 Average AE weight versus average engine power-RPM ratio of I-type AE 
Table 18 Summary of correlation coefficients

\begin{tabular}{|c|c|r|r|r|r|r|r|r|}
\hline \multirow{2}{*}{$\begin{array}{c}\text { Figure } \\
\text { No. }\end{array}$} & \multirow{2}{*}{ Variables } & $y=\mathrm{m}^{*} x+c$ & $y=\mathrm{m}^{*} x$ & $y=\mathrm{m}^{*} x^{\mathrm{n}}$ & $y=\mathrm{m}^{*} \mathrm{e}^{\mathrm{bx}}$ & $y=\mathrm{a}^{*} x^{2}+\mathrm{b}^{*} x+\mathrm{c}$ & $y=\mathrm{a} * \ln (x)+\mathrm{c}$ \\
\cline { 3 - 9 } & & \multicolumn{6}{|c|}{ Values of correlation coefficients $\left(r^{2}\right)$} \\
\hline 60 & $W$ vs $T$ & 0.8524 & 0.8180 & 0.9594 & 0.4590 & 0.9283 & 0.5941 \\
\hline 61 & $W$ vs $T$ & 0.8114 & 0.5955 & 0.9575 & 0.6596 & 0.9213 & 0.9352 \\
\hline
\end{tabular}

\subsubsection{Power-RPM ratio per cylinder}

Auxiliary engine weights are analyzed against the power-RPM ratio per cylinder to determine the AE weight's behaviour from different viewpoints and presented in Figs.62-63. Figure 62 demonstrates the AE weight (observed values) trend against the power-RPM ratio per cylinder. Figure 63 illustrates the behaviour of average AE weight (average by power-RPM ratio per cylinder group) against average power-RPM ratio per cylinder. It also shows the sign of linearity among variables. This shows that with the rise in power-RPM ratio per cylinder, the AE weight increases linearly. Therefore, it is expected that with the increase of power-RPM ratio per cylinder, the AE weight would be increased too.

Figures are additionally investigated for other forms of relationships, and the results are presented in Table 19. Table 19 shows the correlation coefficients under different equation forms. It suggests that the power form yields the highest correlation coefficients for Fig. 62 and 63 but yields from linear and polynomial (second-order) type are not very far from the highest values.

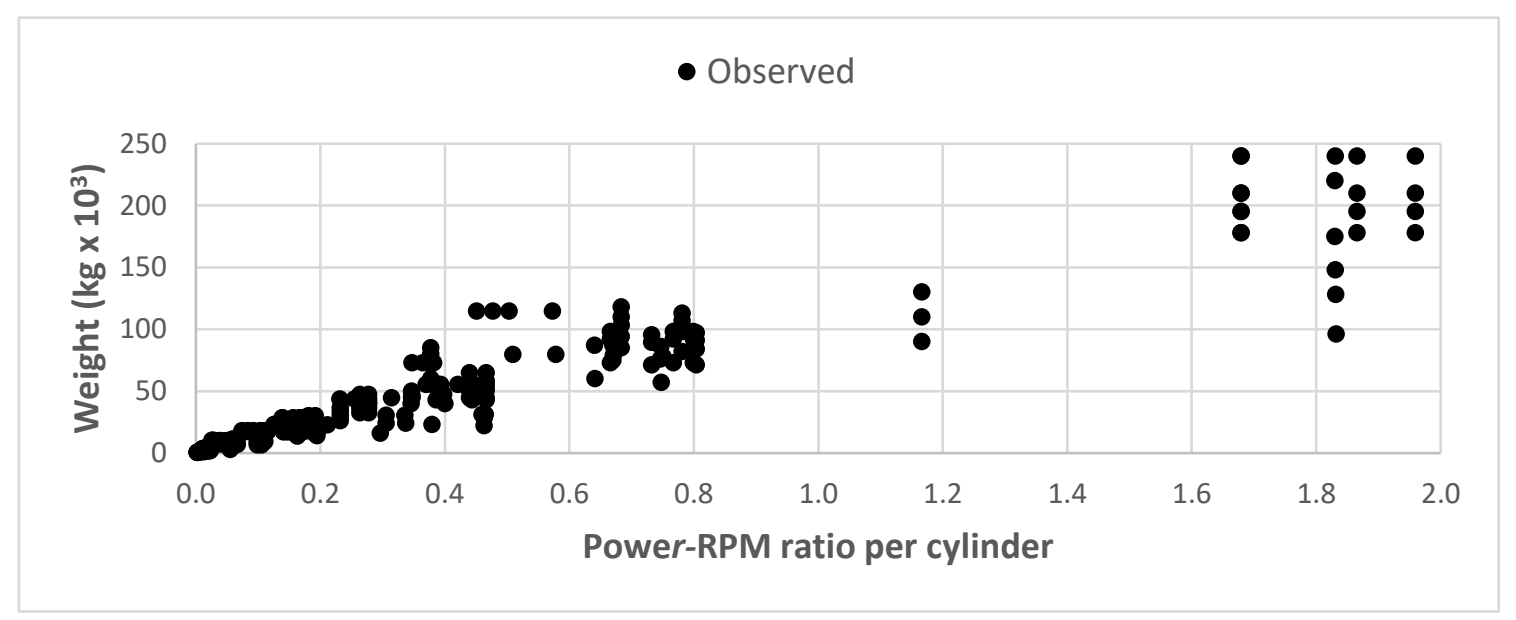

Fig.62 AE weight versus power-RPM ratio per cylinder of I-type $A E$ 


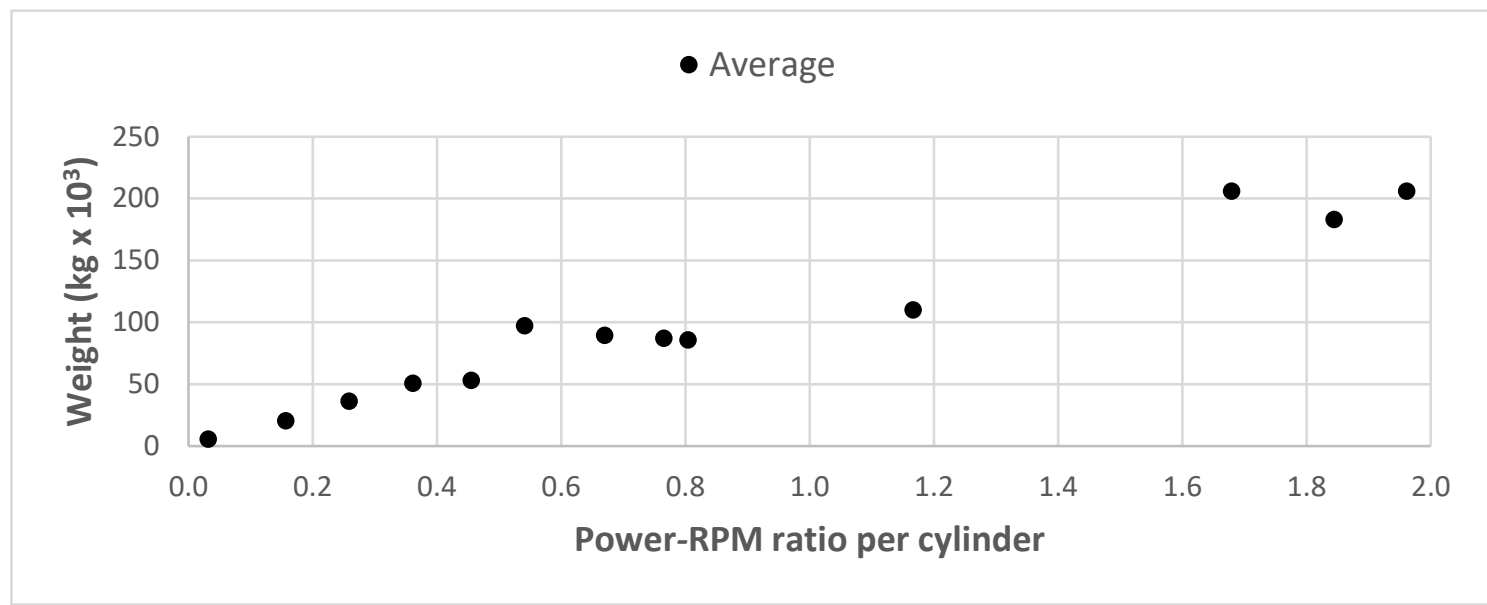

Fig.63 Average AE weight versus average power-RPM ratio per cylinder of I-type AE

Table 19 Summary of correlation coefficients

\begin{tabular}{|c|c|r|r|r|r|r|r|r|}
\hline \multirow{2}{*}{$\begin{array}{c}\text { Figure } \\
\text { No. }\end{array}$} & \multirow{2}{*}{ Variables } & $y=\mathrm{m}^{*} x+\mathrm{c}$ & $y=\mathrm{m}^{*} x$ & $y=\mathrm{m}^{*} x^{\mathrm{n}}$ & $y=\mathrm{m}^{*} \mathrm{e}^{\mathrm{b} x}$ & $y=\mathrm{a} * x^{2}+\mathrm{b}^{*} x+\mathrm{c}$ & $y=\mathrm{a} * \ln (x)+\mathrm{c}$ \\
\cline { 3 - 8 } & & \multicolumn{7}{|c|}{ Values of correlation coefficients $\left(r^{2}\right)$} \\
\hline 62 & $W$ vs $T / N$ & 0.9090 & 0.9034 & 0.9516 & 0.5457 & 0.9138 & 0.5925 \\
\hline 63 & $W$ vs $T / N$ & 0.9534 & 0.9412 & 0.9809 & 0.6997 & 0.9550 & 0.7552 \\
\hline
\end{tabular}

Table 20 is the summary of Tables 15-19. It shows an overview of the highest correlation coefficients of different relationships under different equation forms. It is clear from the table that the power form yields the best goodness fit for a weight-power relationship (observed and average, Figs.54-55 respectively), weight-RPM relationship (observed and average, Figs.56-57 respectively), weightcylinder number relationship (observed, Fig.58), weight-power-RPM ratio relationship (observed and average, Figs.60 and 61 respectively) and weight-power-RPM per cylinder relationship (observed and average, Figs.62 and 63 respectively). Similarly, for weight-cylinder number relationship (average, Fig.59), polynomial form (second-order) produces the best goodness of fit.

Table 20 Summary of highest correlation coefficients

\begin{tabular}{|c|c|c|c|c|c|c|c|c|}
\hline \multirow{2}{*}{$\begin{array}{c}\text { Table } \\
\text { No. }\end{array}$} & \multirow{2}{*}{$\begin{array}{c}\text { Figure } \\
\text { No. }\end{array}$} & \multirow{2}{*}{ Variables } & $y=m^{*} x+c$ & $y=m^{*} x$ & $y=m^{*} x^{n}$ & $y=m^{*} e^{b x}$ & $y=a * x^{2}+b * x+c$ & $y=a * \ln (x)+c$ \\
\hline & & & \multicolumn{6}{|c|}{ Correlation coefficients $\left(r^{2}\right)$} \\
\hline \multirow{2}{*}{15} & 54 & $W$ vs $P$ & & & 0.9562 & & & \\
\hline & 55 & $W$ vs $P$ & & & 0.9662 & & & \\
\hline \multirow{2}{*}{16} & 56 & $W$ vs $R P M$ & & & 0.8260 & & & \\
\hline & 57 & $W$ vs $R P M$ & & & 0.9510 & & & \\
\hline \multirow{2}{*}{17} & 58 & $W$ vs $N$ & & & 0.4077 & & & \\
\hline & 59 & $W$ vs $N$ & & & & & 0.8033 & \\
\hline \multirow{2}{*}{18} & 60 & $W$ vs $T$ & & & 0.9594 & & & \\
\hline & 61 & $W$ vs $T$ & & & 0.9575 & & & \\
\hline \multirow{2}{*}{19} & 62 & $W$ vs $(T / N)$ & & & 0.9516 & & & \\
\hline & 63 & $W$ vs $(T / N)$ & & & 0.9809 & & & \\
\hline
\end{tabular}




\subsection{Multiple linear regression analysis}

Multiple linear regression analysis is a mathematical method used to determine the mathematical relationship involving more than one independent variable, unlike a single independent variable in simple linear regression analysis. This method uses the past data of both dependent and independent variables to establish a relationship to predict the dependent variable against a set of independent variables. A general but comprehensive description of the multiple linear regression analysis is available in Dev, A.K. and Saha, M. $(2015,2016)$.

For this research, the following multiple linear regression model is chosen to represent the relationship expressed in equations 6 and 7 .

$W_{\mathrm{ME}}=b_{0}+b_{1} * P+b_{2} * N+b_{3} * R P M$

$W_{\mathrm{AE}}=b_{0}+b_{1} * P+b_{2} * N+b_{3} * R P M$

Where $W_{\mathrm{ME}}$ is the main engine weight (tonne $=\mathrm{kg} \times 10^{3}$ )

$W_{\mathrm{AE}}$ is the auxiliary engine weight (tonne $=\mathrm{kg} \times 10^{3}$ )

$P$ is the power output $(\mathrm{kW})$ of the main engine (ME) at $100 \%$ MCR and

electrical power output $(\mathrm{kW})$ of the auxiliary engine $(\mathrm{AE})$

$N$ is the cylinder number of engine

$R P M$ is the speed of the engine (revolution per minute)

By the least-squares method (Walpole, R.E. and Myers, R.H., 1978) the required simultaneous equations with unknown regression coefficients are obtained (Dev, A.K. and Saha, M. 2015, 2016). Using the observed data of $\mathrm{W}_{\mathrm{ME}}, \mathrm{W}_{\mathrm{AE}}, \mathrm{P}, \mathrm{RPM}, \mathrm{N}$, the values of the required statistical notations in simultaneous equations are calculated and inserted into the simultaneous equations. The solution of These simultaneous equations' solution yields the regression coefficients' estimate, and the required regression equation is formed. Subsequently, the statistical testing parameters such as i) standard deviation, ii) coefficient of multiple determination and iii) $F$ statistic and its critical value at $5 \%$ significance level, are calculated to demonstrate the model's adequacy.

The same method was applied for the analysis of main engine weight under various speed groups (low-, medium- and high-speed engines)

\subsection{Discussion}

\subsection{General}

There are some significant findings of various V-type and I-type marine propulsion engines' parameters, and auxiliary engines produced globally based on collected data presented in Tables 21 and 22, respectively. Parameters are cylinder number, RPM, power output, and weight. From Table 21 , one can understand that the range of mentioned parameters are significantly different, and it is unfair to compare those by specified parameters. Similarly, from Table 22, one can understand that 
the range of mentioned parameters are significantly different except RPM, and it is unfair to compare those by specified parameters.

Table 21 Summary of the range of values of parameters of V-type and I-type main engines

\begin{tabular}{|c|l|r|r|}
\hline Serial no. & \multicolumn{1}{|c|}{ Item } & \multicolumn{1}{c|}{ V-type } & \multicolumn{1}{c|}{ I-type } \\
\hline 1 & Cylinder number & $8 \sim 20$ & $4 \sim 16$ \\
\hline 2 & RPM & $500 \sim 2,300$ & $60 \sim 2,800$ \\
\hline 3 & Power output (kW) & $492 \sim 21,600$ & $93 \sim 82,440$ \\
\hline 4 & ME weight (tonne) & $3 \sim 265$ & $1 \sim 2,400$ \\
\hline
\end{tabular}

Table 22 Summary of the range of values of parameters of

V-type and I-type auxiliary engines

\begin{tabular}{|c|l|r|r|}
\hline Serial no. & \multicolumn{1}{|c|}{ Item } & \multicolumn{1}{c|}{ V-type } & \multicolumn{1}{c|}{ I-type } \\
\hline 1 & Cylinder number & $8 \sim 20$ & $3 \sim 20$ \\
\hline 2 & RPM & $514 \sim 1,800$ & $514 \sim 1,800$ \\
\hline 3 & Power output (kW) & $600 \sim 16,111$ & $12 \sim 16,940$ \\
\hline 4 & AE weight (tonne) & $0.5 \sim 345$ & $0.5 \sim 240$ \\
\hline
\end{tabular}

\subsection{Marine propulsion machinery}

Weights are analyzed against power output (Figs.14-15, 24-25, 34-35, 44-45), RPM (Figs.16-17, 26-27, 36-37, 46-47), cylinder number (Figs.18-19, 28-29, 38-39, 48-49), power-RPM ratio (Figs.20-21, 30-31, 40-41, 50-51), power-RPM ratio per cylinder (Figs.22-23, 32-33, 42-43, 52-53) under combined and speed groups (low-speed, medium-speed and high-speed) and the correlation coefficients of corresponding relationships under various forms are presented in Tables 5-9 and 11-13 respectively. Detailed explanations regarding the nature of the relationship of competing variables are given in SubSub-Sections 5.1.1, 5.1.2, 5.1.3, 5.1.4, 5.1.5, 5.1.6.1, 5.1.6.2, and 5.1.6.3, respectively. All these figures suggest that the weight of a marine diesel engine is very much dependent on power output, RPM, cylinder number, power-RPM ratio, and power-RPM ratio per cylinder, but of different magnitudes and trends. Hence, the weight is a function of power output, RPM, cylinder number, power-RPM ratio, and power-RPM ratio per cylinder exactly, as per earlier assumptions. However, the change in weight against the variation in the afore-mentioned variables is different in terms of magnitude and behaviour. Table 10 summarizes the highest correlation coefficients for different figures under a different form of equations. One can find that for a weight-power relationship (observed, Fig.14), weight-cylinder number relationship (observed and average, Figs.18 and 19 respectively), a polynomial form (second-order) yields the best goodness of fit. Similarly, for weight-power relationship (average, Fig.15), weight-RPM relationship (observed and average, Figs.16 and 17 respectively), weight-power-RPM ratio relationship (observed and average, Figs.20 and 21 
respectively) and weight-power-RPM ratio per cylinder (observed and average, Figs.22 and 23 respectively), a power form yields the best goodness of fit, and so on.

Table 14 provides a summary of the highest correlation coefficient values for different forms under various speed groups. One can find that the speed group yields better results than the combined group for low and medium-speed engines. Power-RPM ratio relation produces the highest values. It shows that the models have a better fit when main engines are dealt as per speed groups, power-RPM ratio as independent variable and power form relationship with weight.

Using a mathematical model under the estimation method of least squares and collected data, the final regression equation for the marine propulsion engine weight (tonne $=\mathrm{kg} \times 10^{3}$ ) under combined and speed groups (low, medium and high) are as follows respectively:

$W_{\mathrm{ME}}=23.0684+0.0343 * P-8.3134 * N-0.0236 * R P M$

$W_{\mathrm{ME}}=114.3358+0.0321 * P+1.0762 * N-1.1504 * R P M$, where $R P M \leq 400$

$W_{\mathrm{ME}}=33.236+0.0147 * P-2.88463 * \boldsymbol{N}-0.0257 * R P M$, where $400 \leq R P M \leq 1000$

$W_{\mathrm{ME}}=-2.3965+0.003 * P+1.457 * N-0.0024 * R P M, R P M \geq 1000$

In the regression equations, power output and RPM have similar signs as assumed, but cylinder number has the opposite sign for the combined and medium-speed group. However, the findings are still in line with the assumptions made earlier, except for the cylinder number.

Table 23 displays the regression coefficients' estimates and other statistical parameters of the regression equations while adding new variables. From the table, one can understand that the successful inclusion of variables of the main engine parameters in the model contributed to a higher $R^{2}$ value, which is the primary measurement of the model's adequacy.

Table 23 Values of parameters of different mathematical models for main engines

Combined group

\begin{tabular}{|c|c|c|c|c|c|c|c|c|c|c|c|c|c|}
\hline \multirow{2}{*}{$\begin{array}{c}\text { Mathematical } \\
\text { models }\end{array}$} & \multicolumn{8}{|c|}{ Regression coefficients } & \multicolumn{5}{|c|}{ Statistical parameters } \\
\hline & $n$ & $k$ & $a$ & $b$ & $b_{0}$ & $b_{1}$ & $b_{2}$ & $b_{3}$ & $S$ & $R^{2}$ & $f$ & $f_{0.05}$ & $f-f_{0.05}$ \\
\hline$W_{\text {ENGINE }}=f(P)$ & 3,006 & 1 & -33.26 & 0.03 & NA & NA & NA & NA & 118 & 0.9298 & 39,766 & 3.84 & 39,762 \\
\hline$W_{\text {ENGINE }}=f(P, N)$ & 3,006 & 2 & NA & NA & 22.75 & 0.03 & -9.35 & NA & 118 & 0.9304 & 20,091 & 3.00 & 20,088 \\
\hline $\begin{array}{l}W_{\text {ENGINE }}= \\
f(P, N, R P M)\end{array}$ & 3,006 & 3 & NA & NA & 23.07 & 0.03 & -8.31 & -0.02 & 118 & 0.9307 & 13,440 & 2.60 & 13,437 \\
\hline
\end{tabular}

Low-speed group

\begin{tabular}{|l|c|c|c|c|c|c|c|c|c|c|c|c|c|}
\hline$W_{\text {ENGINE }}=f(P)$ & 2,698 & 1 & -26.69 & 0.03 & NA & NA & NA & NA & 123 & 0.9298 & 34,143 & 3.84 & 34,139 \\
\hline $\begin{array}{l}W_{\text {ENGINE }}= \\
f(P, R P M)\end{array}$ & 2,698 & 2 & NA & NA & 119.56 & 0.03 & -1.14 & NA & 117 & 0.9335 & 18,928 & 3.00 & 18,925 \\
\hline $\begin{array}{l}W_{\text {ENGINE }}= \\
f(P, N, R P M)\end{array}$ & 2,698 & 3 & NA & NA & 114.34 & 0.03 & 1.08 & -1.15 & 117 & 0.9335 & 12,620 & 2.60 & 12,617 \\
\hline
\end{tabular}

Medium-speed group

\begin{tabular}{|l|c|c|c|c|c|c|c|c|c|c|c|c|c|}
\hline$W_{\text {ENGINE }}=f(P)$ & 255 & 1 & -8.09 & 0.02 & NA & NA & NA & NA & 9 & 0.9514 & 4,958 & 3.84 & 4,954 \\
\hline$W_{\text {ENGINE }}=f(P, N)$ & 255 & 2 & NA & NA & 16.56 & 0.02 & -3.79 & NA & 8 & 0.9668 & 3,681 & 3.00 & 3,678 \\
\hline
\end{tabular}




\begin{tabular}{|l|c|c|c|c|c|c|c|c|c|c|c|c|c|}
$\begin{array}{l}W_{\mathrm{ENGINE}}= \\
f(P, N, R P M)\end{array}$ & 255 & 3 & $\mathrm{NA}$ & $\mathrm{NA}$ & 33.24 & 0.01 & -2.85 & -0.03 & 7 & 0.9706 & 2,783 & 2.60 & 2,780 \\
\hline
\end{tabular}

High-speed group

\begin{tabular}{|l|c|c|c|c|c|c|c|c|c|c|c|c|c|}
\hline$W_{\text {ENGINE }}=f(N)$ & 53 & 1 & -15.40 & 3.07 & NA & NA & NA & NA & 2 & 0.6697 & 41 & 4.04 & 37 \\
\hline $\begin{array}{l}W_{\text {ENGINE }}= \\
f(N, R P M)\end{array}$ & 53 & 2 & NA & NA & -4.03 & 2.21 & 0.00 & NA & 2 & 0.6132 & 40 & 3.19 & 37 \\
\hline $\begin{array}{l}W_{\text {ENGINE }}= \\
f(P, N, R P M)\end{array}$ & 53 & 3 & NA & NA & -2.40 & 0.003 & 1.46 & 0.00 & 2 & 0.6435 & 31 & 2.80 & 28 \\
\hline
\end{tabular}

NA : Not Applicable

Focusing on the final regression equations, which pass the $F$ statistic test, the value of the multiple determinations' coefficient is $0.9307,0.9335,0.9706$ and 0.6435 for the combined, low-, medium- and high-speed groups respectively, which can be considered very high except for low-speed group. Mathematically, this means that providing the example for the combined group, $93 \%$ of the variation in the dependent variable (ME weight) is contributed due to the difference in the independent variables and the remaining (7\%) is called the error of estimation. Statistically, it is called an error of the sum of squares (SSE) or unexplained variation. This variation behaves randomly or unpredictably (Murray, R.S. 1992). It also reflects the difference in the regression line. However, this estimation error is due to the absence of one or more critical independent variables responsible for the change in the dependent variable. The coefficient of multiple determination and the error of estimation are inversely related, meaning that for the higher value of the coefficient of multiple determination, the error of estimation would be low and vice versa. The lower value of the coefficient may also occur if the collected data suffers inconsistency, meaning that some of the independent variables have low values (Dev, A.K. and Saha, M. 2015).

Validation of a postulated mathematical model is an alternative way to demonstrate the model's adequacy of fitness to the system in question. To carry out the mathematical model's validation (Equations 10, 11, 12 and 13), each model is applied to estimate $W_{\mathrm{ME}}$ for each main engine and compared with actual data of main engine weight regarding deviation (\% error). The summary of the result is presented in Table 24. The table shows the outline of the variation of model values from the

Table 24 Summary of validation results

\begin{tabular}{|r|r|r|r|r|}
\hline Regression Equation & Combined & Low-Speed & Medium-Speed & High-Speed \\
\hline$R^{2}$ & 0.9307 & 0.9335 & 0.9706 & 0.6435 \\
\hline Maximum error (\%) & 127 & 66 & 53 & 189 \\
\hline Minimum error (\%) & -13946 & $-1,936$ & -139 & -74 \\
\hline Range (\%) & 14,072 & 2,002 & 192 & 263 \\
\hline Mean (\%) & -61.16 & -6 & -5 & 33 \\
\hline Variance & 453,849 & 8,458 & 960 & 3,044 \\
\hline Standard Deviation & 674 & 92 & 31 & 55 \\
\hline
\end{tabular}


actual values. The table shows that the range of error is very high for all models, more on the negative side, except for the high-speed group. This means that models yield a wide range of error, which is not acceptable. This also suggests that the distribution of error has a backward skew. Due to this wide range of error, the proposed multiple linear regression model does not appropriately explain the data. The possible reasons are described as follows.

The "Forward selection" method under the "stepwise regression" technique ensures the most useful variables from a set of variables. However, it is quite possible that a variable entering the regression equation at an early stage might have been rendered unimportant or redundant because of relationships that exist with other variables entering later stages. When the $F$ value exceeds the tabulated critical point of the $F$ distribution for the degrees of freedom of the samples at a selected significance level [i.e., $f>f_{\alpha}(k, n-k-1)$ ], it does indicate that the regression explained by the model is significant, but this does not rule out the possibility that

(a) The selected linear regression model with the selected set of variables is not the only model that can be used to explain the data; indeed, there might be other models with transformations on existing variables that might give a more significant value of the $F$ statistic (Walpole, R.E and Myers, R.H. 1978). In this case, power or polynomial form of the equation and power-RPM ratio or power-RPM ratio per cylinder as independent variables might yield the more significant $F$ statistic.

(b) The model might have been more productive with the inclusion of other variables in addition to existing variables, or perhaps the deletion of one or more of the variables in the model (Walpole, R.E and Myers, R.H. 1978). In this case, cylinder bore diameter, stroke length, etc. could be the additional variables. However, this is beyond the scope of this article.

Moreover, detailed investigations reveal that excessive negative deviations occur in the case of highspeed main engines. Mathematically, this is very much expected, due to the negative contribution of speed in final regression equations of all groups (equations 10-13). It is clear from Table 24 that with high $R^{2}$ value, the model could yield an extensive range of error. With this outcome, it can be concluded that the multiple linear regression model, combined or separate speed group, does not provide sufficient adequacy to fit the data. Furthermore, based on the mean value of power output, cylinder number and RPM of sample engines, low-speed, medium-speed, and high-speed engines, their contributions to the mean ME weight are calculated and presented in Table 25. If any combinations of independent variables deviate from this order, the model will yield the different model value depending on the combination's equivalent mean value. 
Table 25 Comparison of contribution by variables

\begin{tabular}{|l|r|r|r|r|}
\cline { 2 - 5 } \multicolumn{1}{c|}{} & \multicolumn{4}{c|}{ Components' contribution (\%) } \\
\hline Components $\downarrow$ & Combined & Low-Speed & Medium-Speed & High-Speed \\
\hline Constant $\left(b_{0}\right)$ & 4.43 & 16.58 & 25.29 & 13.63 \\
\hline Power output $(P)$ & 83.72 & 64.08 & 44.74 & 9.03 \\
\hline Cylinder number $(N)$ & 10.97 & 1.07 & 15.99 & 51.28 \\
\hline Speed $(\mathrm{RPM})$ & 0.87 & 18.28 & 13.98 & 26.06 \\
\hline
\end{tabular}

Referring to the statement made in point (a) above, various equations, such as power form, polynomial form and transformed form of existing independent variables, such as power-RPM ratio and power-RPM ratio per cylinder are tried, including validation. The results are presented in Table 26. One can identify that the power form and transformed independent variables (power-RPM ratio and power-RPM ratio per cylinder) yield the comparatively better fit to data regarding deviation from an actual value.

At this point of discussion, one should remember that the mean error (\%) is just a figure, and it must not be the criteria of selection of the model. Instead, the range of error (\%) should be the criteria. It indicates the stretch of error. Under power form, Figs.23 and 21 have mean errors of 2 and -3 respectively, whereas their range of error is 202 (-89 to 113) and 139 (-87 to 52) respectively. Fig. 21 is more suitable than Fig.23 for estimation purposes.

Table 26 Validation results of other models

\begin{tabular}{|r|r|r|r|r|r|r|}
\hline Equations $\rightarrow$ & \multicolumn{3}{|c|}{ Power form } & \multicolumn{3}{c|}{ Polynomial form } \\
\hline Figures $\rightarrow$ & \multicolumn{1}{|c|}{15} & \multicolumn{1}{c|}{21} & \multicolumn{1}{c|}{23} & \multicolumn{1}{c|}{15} & \multicolumn{1}{c|}{21} & \multicolumn{1}{c|}{23} \\
\hline$r^{2}$ value $\rightarrow$ & 0.9798 & 0.9955 & 0.9838 & 0.9743 & 0.9895 & 0.9783 \\
\hline Maximum Error (\%) & 735 & 52 & 113 & 228 & 56 & 119 \\
\hline Minimum Error (\%) & -52 & -87 & -89 & $-20,494$ & $-4,946$ & $-7,547$ \\
\hline Range (\%) & 787 & 139 & 202 & 20,722 & 5,002 & 7,666 \\
\hline Mean (\%) & 10 & -3 & 2 & 136 & -56 & -74 \\
\hline Variance & 3,521 & 465 & 1,023 & $1,168,836$ & 81,164 & 187,667 \\
\hline Standard Deviation & 59 & 22 & 32 & 1,081 & 285 & 433 \\
\hline
\end{tabular}

Table 27 shows a summary of the validation results of different relationships under a different form of equations and speed groups. According to the table and based on the range of error, $W=\mathrm{a}^{*} T^{\mathrm{n}}$ (power form) provides the best fit for all engines, low and medium-speed engines, and $W=\mathrm{a} * T^{2}+\mathrm{b}$ * $T+\mathrm{c}$ (polynomial-second-order) provides an excellent fit to high-speed engines. 
Table 27 Summary of validation result under different forms and speed groups

Combined main engines

\begin{tabular}{|r|r|r|r|r|r|r|}
\cline { 2 - 7 } \multicolumn{1}{c|}{} & \multicolumn{3}{c|}{ Figure $21,[W=f(T)]$} & \multicolumn{3}{c|}{ Figure 23, $[W=f(T / N)]$} \\
\hline Item & Linear & Power & Polynomial & Linear & Power & Polynomial \\
\hline Maximum error (\%) & 24,790 & 52 & 56 & 115 & 113 & 119 \\
\hline Minimum error (\%) & -42 & -87 & $-4,946$ & $-1,683$ & -89 & $-7,547$ \\
\hline Range & 24,832 & 139 & 5,002 & 1,798 & 202 & 7,666 \\
\hline Mean & 236 & -3 & -56 & -19 & 2 & -74 \\
\hline Variance & $1,970,466$ & 465 & 81,164 & 10,775 & 1,023 & 187,667 \\
\hline Standard dev. & 1,404 & 22 & 285 & 104 & 32 & 433 \\
\hline
\end{tabular}

Low-speed engines

\begin{tabular}{|r|r|r|r|r|r|r|}
\cline { 2 - 7 } \multicolumn{1}{c|}{} & \multicolumn{2}{c|}{ Figure $31,[W=f(T)]$} & \multicolumn{3}{c|}{ Figure 33, $[W=f(T / N)]$} \\
\hline Item & Linear & Power & Polynomial & Linear & Power & Polynomial \\
\hline Maximum error (\%) & 859 & 51 & 56 & 116 & 53 & 117 \\
\hline Minimum error (\%) & -42 & -50 & -250 & -196 & -75 & -241 \\
\hline Range & 901 & 101 & 306 & 313 & 128 & 358 \\
\hline Mean & 50 & 1 & -13 & -5 & -18 & -7 \\
\hline Variance & 4,470 & 247 & 777 & 965 & 387 & 1,104 \\
\hline Standard dev. & 67 & 16 & 28 & 31 & 20 & 33 \\
\hline
\end{tabular}

Medium-speed engines

\begin{tabular}{|r|r|r|r|r|r|r|}
\cline { 2 - 7 } \multicolumn{1}{c|}{} & \multicolumn{3}{c|}{ Figure $41,[W=f(T)]$} & \multicolumn{3}{c|}{ Figure 43, $[W=f(T / N)]$} \\
\hline Item & Linear & Power & Polynomial & Linear & Power & Polynomial \\
\hline Maximum error (\%) & 66 & 49 & 38 & -7 & 82 & 75 \\
\hline Minimum error (\%) & -23 & -25 & -51 & -87 & -50 & -50 \\
\hline Range & 89 & 74 & 89 & 81 & 132 & 126 \\
\hline Mean & 7 & 1 & -4 & -67 & 5 & 4 \\
\hline Variance & 306 & 173 & 214 & 327 & 493 & 465 \\
\hline Standard dev. & 17 & 13 & 15 & 18 & 22 & 22 \\
\hline
\end{tabular}

High-speed engines

\begin{tabular}{|r|r|r|r|r|r|r|}
\cline { 2 - 7 } \multicolumn{1}{c|}{} & \multicolumn{3}{c|}{ Figure $51,[W=f(T)]$} & \multicolumn{3}{c|}{ Figure 53, $[W=f(T / N)]$} \\
\hline Item & Linear & Power & Polynomial & Linear & Power & Polynomial \\
\hline Maximum error (\%) & 166 & 118 & 129 & 103 & 121 & 97 \\
\hline Minimum error (\%) & -68 & -75 & -171 & -78 & -74 & -76 \\
\hline Range & 234 & 192 & 300 & 181 & 195 & 174 \\
\hline Mean & 52 & 21 & 12 & 11 & 20 & 19 \\
\hline Variance & 4,260 & 2,289 & 3,948 & 2,283 & 2,581 & 2,326 \\
\hline Standard dev. & 65 & 48 & 63 & 48 & 51 & 48 \\
\hline
\end{tabular}

Note: Linear: $y=m^{*} x+c$, Power: $y=m^{*} x^{n}$, Polynomial: $y=a^{*} x^{2}+b^{*} x+c$

Typically, when the values of independent variables are closer to the sample's mean value, the model would yield a reasonable estimate of negligible deviation. This can be demonstrated in Figure 64 ( $\mu$ refers to the equivalent mean values of various independent variables of sample data). The Figure 
shows that the independent variables' values lower than the sample data's equivalent mean value increased the estimated engine weight giving a higher positive deviation. Inversely, the independent variables' values more elevated than the equivalent mean value of the sample data resulted in a decrease in the estimated engine weight giving a higher negative deviation. This phenomenon is due to RPM's negative contribution in final regression equations, and it is very much expected. However, someone may alternatively choose to use the individual relationship of the independent variables with the dependent variable.

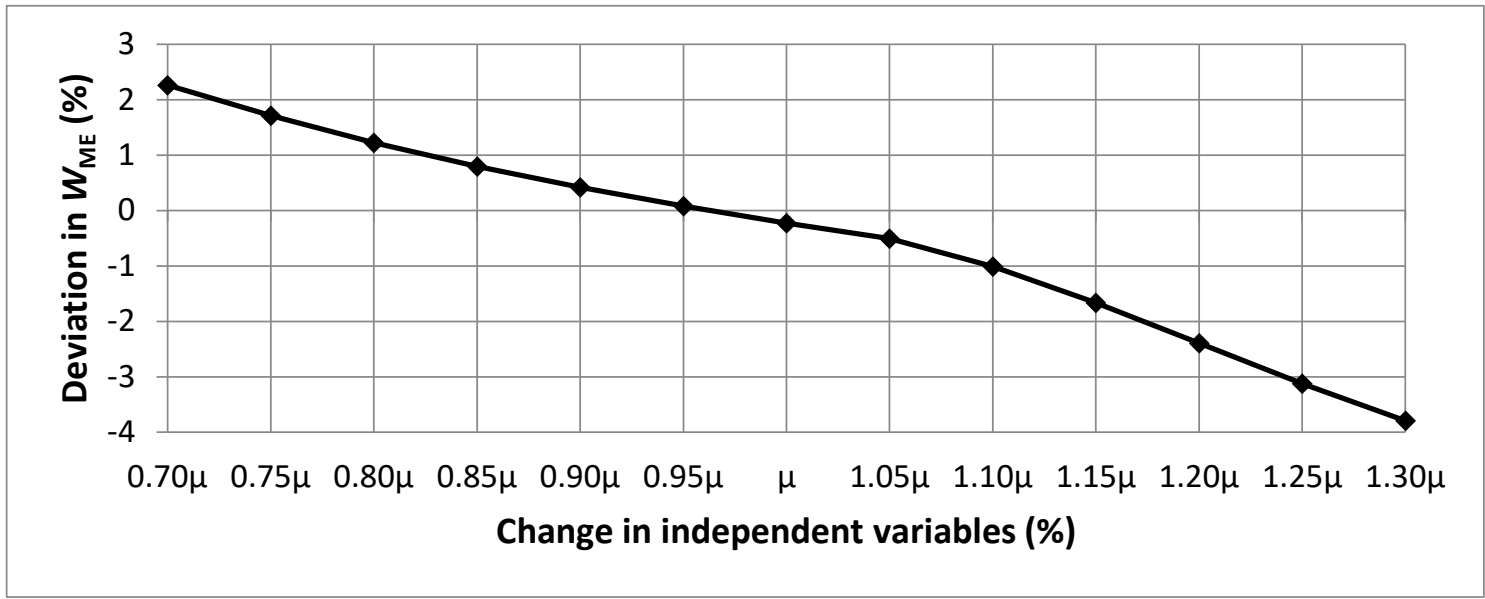

Fig.64 Deviation in $W_{\mathrm{ME}}(\%)$ versus change in the equivalent mean value of independent variables (\%)

Finally, the proposed mathematical model (multiple linear regression equation) may be used to estimate engine weight for initial stability calculation during the preliminary and conceptual design stage. Using the above model as a guide, designers may estimate the expected engine weight against expected main engine parameters. If the desired independent variables are close to the mean value (Table 1), the model will provide a reasonable estimate of engine weight. The assessment could be high and low ( $+3 \%$ to $-4 \%)$ accordingly. Alternatively, one may choose to use the individual relationship under different equation form and other speed groups as appropriate.

\subsection{Power generation machinery}

Weights are analyzed against power output (Figs.54-55), RPM (Figs.56-57), cylinder number (Figs.5859), power-RPM ratio (Figs.60-61), the power-RPM ratio per cylinder (Figs.62-63) and the correlation coefficients of corresponding relationships under various forms are presented in Tables 15-19, respectively. Detailed explanations regarding the nature of the relationship of competing variables are given in Sub-Sub-Sections 5.2.1, 5.2.2, 5.2.3, 5.2.4, and 5.2.5, respectively. All these figures suggest that the AE weight (generator coupled with a diesel engine) is very much dependent on power output, RPM, cylinder number, power-RPM ratio, and power-RPM ratio per cylinder, but of different magnitudes and trends. Hence, the AE weight is a function of power output, RPM, cylinder number, 
power-RPM ratio and power-RPM ratio per cylinder, precisely as per assumption. However, the change in $\mathrm{AE}$ weight against the difference in the mentioned variables is different in terms of magnitude and behaviour. Table 20 provides the highest correlation coefficients for other figures under a different form of equations. One can easily find that for a weight-power relationship (observed and average, Figs.54 and 55 respectively), weight-RPM relationship (observed and average, Figs.56 and 57 respectively), weight-cylinder number relationship (observed, Fig.58), weight-powerRPM ratio relationship (observed and average, Figs. 60 and 61 respectively) and power-RPM ratio per cylinder relationship (observed and average, Figs.62 and 63 respectively), power form yields the best goodness of fit. Similarly, for weight-cylinder number (average, Fig.59) polynomial form (secondorder) generates the best goodness of fit.

Using a mathematical model under the estimation method of least squares and collected data, the final regression equation for the marine auxiliary engine weight (tonne) is as follows:

$W_{\mathrm{AE}}=57.0056+0.0225 * P-5.6727 * N-0.0168 * R P M$

In the regression equation, power output has a similar sign as assumed, but cylinder number and RPM have the opposite sign. The findings are otherwise still in line with the assumptions made earlier, except for the cylinder number and RPM.

Table 28 displays the regression coefficients' estimates and other statistical parameters of the regression equation while adding new variables. From the table, one can easily understand that the successful inclusion of variables of the auxiliary engine parameters in the model contributed to higher $R^{2}$ value, which is the primary measurement of the model's adequacy.

Table 28 Values of parameters of different mathematical models for auxiliary engines

\begin{tabular}{|c|c|c|c|c|c|c|c|c|c|c|c|c|c|}
\hline \multirow{2}{*}{$\begin{array}{c}\text { Mathematical } \\
\text { model }\end{array}$} & \multicolumn{8}{|c|}{ Regression coefficients } & \multicolumn{5}{|c|}{ Statistical parameters } \\
\hline & $n$ & $k$ & $a$ & $b$ & $b_{0}$ & $b_{1}$ & $b_{2}$ & $b_{3}$ & $S$ & $R^{2}$ & $f$ & $f_{0.05}$ & $f-f_{0.05}$ \\
\hline$W_{\mathrm{AE}}=f(P)$ & 288 & 1 & 3.68 & 0.02 & NA & NA & NA & NA & 21 & 0.8457 & 1,568 & 3.84 & 1,564 \\
\hline$W_{\mathrm{AE}}=f(P, N)$ & 288 & 2 & NA & NA & 35.22 & 0.02 & -5.52 & NA & 19 & 0.8732 & 985 & 3.00 & 982 \\
\hline$W_{\mathrm{AE}}=f(P, N, R P M)$ & 267 & 3 & NA & NA & 57.01 & 0.02 & -5.67 & -0.02 & 19 & 0.8845 & 676 & 2.60 & 673 \\
\hline
\end{tabular}

NA : Not Applicable

Focusing on the final regression equation (14), which passes the $F$ statistic test, the multiple determination coefficient value is 0.88 , which can be considered high. Mathematically, $88 \%$ of the dependent variable (AE weight) variation is contributed due to the difference in the independent variables, and the remaining $(12 \%)$ is called the error of estimation. Statistically, it is called an error of the sum of squares (SSE) or unexplained variation. This variation behaves randomly or unpredictably (Murray, R.S. 1992). It also reflects the interpretation of the regression line. However, this estimation error is due to the absence of one or more influential independent variables responsible for the dependent variable's change. The coefficient of multiple determination and the error of estimation 
are inversely related, meaning that for the higher value of the coefficient of considerable determination, the assessment error would be low and vice versa. The lower value of the coefficient may also occur if the collected data suffers inconsistency, meaning that some of the independent variables have low values (Dev, A.K. and Saha, M. 2015).

The validation procedure, like that applied to the model (equation 10) for ME weight, is applied to the model (equation 14) for AE weight. The result is presented in Table 29. The table shows the summary of deviation of model values from the actual values. The table shows that the range of error is very high, 2721 (-385 t0 2337), especially at the positive side.

Table 29 Summary of validation results

\begin{tabular}{|r|r|}
\hline \multicolumn{1}{|c|}{ Model } & Multiple Linear Regression \\
\hline Maximum error (\%) & 2337 \\
\hline Maximum error (\%) & -385 \\
\hline Range & 2721 \\
\hline Mean & 41 \\
\hline variance & 71263 \\
\hline Standard deviation & 267 \\
\hline
\end{tabular}

This suggests that the distribution of error has a forward skew. Due to this wide range of error, the proposed multiple linear regression model does not explain the data. The possible reasons are precisely similar to those, as explained in Sub-Section 6.2, for ME weight.

Moreover, detailed investigation reveals that the excessive positive deviations occur in low power output with high-speed auxiliary engines (13 kW and 1800 RPM). Mathematically, it is expected due to the high positive intercept $\left(b_{0}=57\right)$ in the final regression equation (14). This situation suggests that the mentioned model (equation 14) cannot explain auxiliary engine weight data.

Furthermore, based on the mean value of power output, cylinder number, and RPM of sample auxiliary engines, their contributions to the mean AE weight are calculated and presented in Table 30. If any combinations of independent variables deviate from this order, the model will yield the different model value depending on the combination's equivalent mean value.

Table 30 Contribution by variables

\begin{tabular}{|l|r|}
\hline Components & Contribution $(\%)$ \\
\hline Constant $\left(b_{0}\right)$ & 35.62 \\
\hline Power output $(P)$ & 29.32 \\
\hline Cylinder number $(N)$ & 24.6 \\
\hline Speed (RPM) & 10.46 \\
\hline
\end{tabular}

Referring to the statement made in point (a), Sub Section 6.2, various models, such as power form, polynomial form and transformed form of existing independent variables, such as power-RPM ratio 
and power-RPM ratio per cylinder are tried, including validation. The results are presented in Table 31. One can identify that the power form and transformed independent variables (power-RPM ratio and power-RPM ratio per cylinder) yield the comparatively better fit to data regarding deviation from an actual value.

Table 31 Summary of validation result under different model

\begin{tabular}{|r|r|r|r|r|r|r|}
\cline { 2 - 7 } \multicolumn{1}{c|}{} & \multicolumn{3}{c|}{ Figure 61 $[W=f(T)]$} & \multicolumn{3}{c|}{ Figure 63 $[W=f(T / N)]$} \\
\hline Item & Linear & Power & Polynomial & Linear & Power & Polynomial \\
\hline Maximum error (\%) & 2,925 & 174 & 390 & 2,810 & 210 & 1,712 \\
\hline Minimum error (\%) & -87 & -49 & -92 & -50 & -55 & $-2,183$ \\
\hline Range & 3,013 & 223 & 483 & 2,860 & 265 & 3,895 \\
\hline Mean & 125 & 5 & -55 & 188 & 16 & 474 \\
\hline Variance & 228,833 & 1,201 & 5,760 & 203,854 & 1,881 & 155,719 \\
\hline Standard deviation & 478 & 35 & 76 & 452 & 43 & 395 \\
\hline
\end{tabular}

Note: Linear: $y=m^{*} x+c$, Power: $y=m^{*} x^{n}$, Polynomial: $y=a^{*} x^{2}+b^{*} x+c$

As explained in the ME weight case, when independent variables are closer to the sample's mean value, the model would yield a reasonable estimate with negligible error. This is equally applicable for AE weight and is demonstrated in Fig.65 ( $\mu$ refers to the equivalent mean value of various independent variables of sample data).

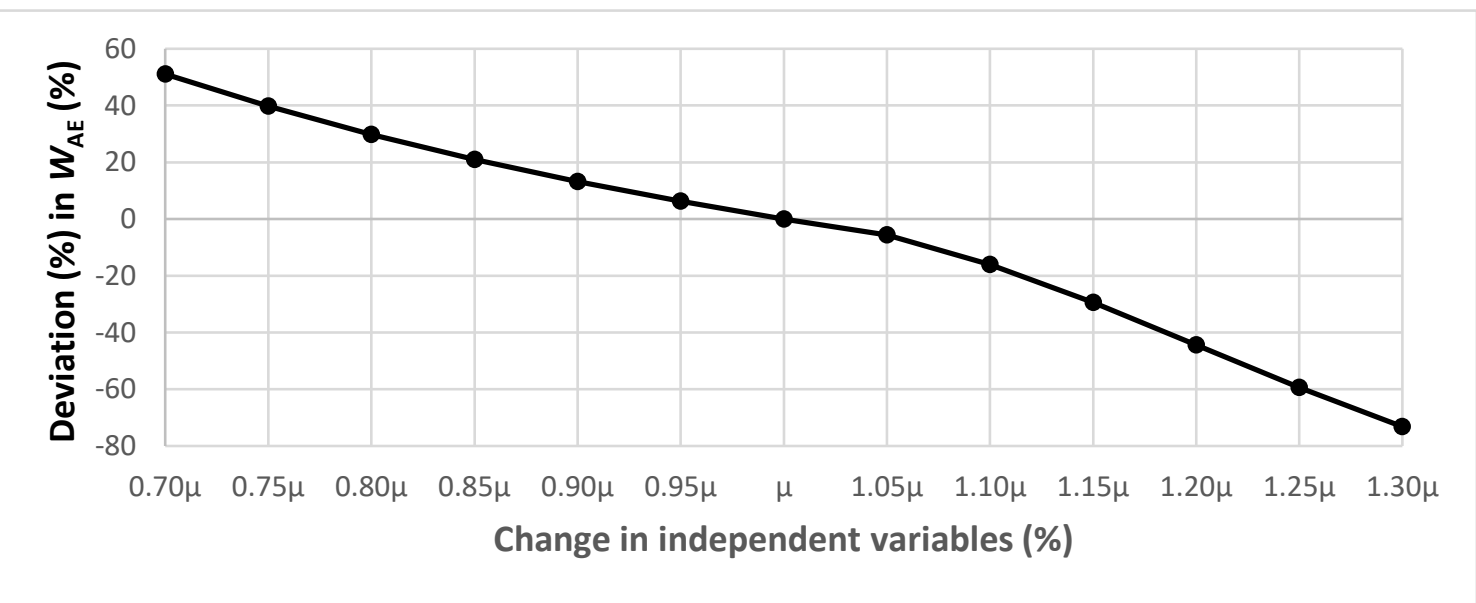

Fig.65 Deviation in $W_{\mathrm{AE}}(\%)$ versus change in equivalent mean value of an independent variable (\%) Finally, the proposed mathematical model may estimate auxiliary engine weight for initial stability calculation during the preliminary and conceptual design stage. Using the above model as a guide, designers may estimate the expected engine weight against expected auxiliary engine parameters. If the desired independent variables are close to the mean value (Table 2), then the model would provide a reasonable estimate of engine weight. However, for low and high values, the calculations could be high and low, respectively.

\subsection{Weight Estimation}




\subsubsection{Main Propulsion Engine}

Table 10 suggests that the weight-power relationship (Fig.15), weight-power-RPM ratio relationship (Fig.21) and weight-power-RPM ratio per cylinder relationship (Fig.23) yield the highest correlation coefficients under power equation form. It is evident from the validation results (Table 24) that the proposed mathematical models (equations $10,11,12$ and 13 ) yield a wide range of errors (\%) and high standard deviation (\%) which will affect the accuracy of the estimation of engine weight. The validation results of various relationships under different equations (Table 26) dictate that the power equation yields the lower range of error (\%) and a smaller standard deviation (\%). Table 32 displays a summary of the above findings. It is very clear from the Table that the above mentioned three relationships under power equation form yield the optimum result in terms of mentioned statistical parameters. During the preliminary and conceptual design stage, the designers have only two options to estimate the engine weight, i) based on engine power (kW), ii) based on engine power (kW) and engine speed (RPM). The cylinder number is not useful at that stage. As such, weight-power (Fig.15) and weightpower-RPM ratio (Fig.21) relationships are the most appropriate to estimate the engine weight.

Table 32 Summary of validation results

\begin{tabular}{|r|r|r|r|}
\cline { 2 - 4 } \multicolumn{1}{c|}{} & $W=\mathrm{a}^{*} P^{\mathrm{n}}$ & $W=\mathrm{a}^{*} T^{\mathrm{n}}$ & $W=\mathrm{a}^{*}(T / N)^{\mathrm{n}}$ \\
\hline Item & \multicolumn{1}{c|}{ Fig.15 } & Fig.21 & \multicolumn{1}{c|}{ Fig.23 } \\
\hline Maximum error (\%) & 735 & 52 & 113 \\
\hline Minimum error (\%) & -52 & -87 & -89 \\
\hline Range (\%) & 787 & 139 & 202 \\
\hline Mean (\%) & 10 & -3 & 2 \\
\hline Variance (\%) & 3,521 & 465 & 1,023 \\
\hline Standard deviation (\%) & 59 & 22 & 32 \\
\hline
\end{tabular}

Accordingly, Fig.66 is developed using the relationship of weight-power output of main engines under the power equation (Fig.15). Figure 66 shows the full range of estimated weight against the full range of estimated power output calculated during the conceptual and preliminary design stage. It shows the relationships between the estimated ME weight and estimated power output of propulsion engine under power equation.

Figure 67 is developed using the relationship of ME weight and a power-RPM ratio under power equations (Fig.21) for various constant RPMs. It is clear from the Fig.99 that the ME weight decreases with RPM's increase for particular power output. This is in line with the initial assumption. 


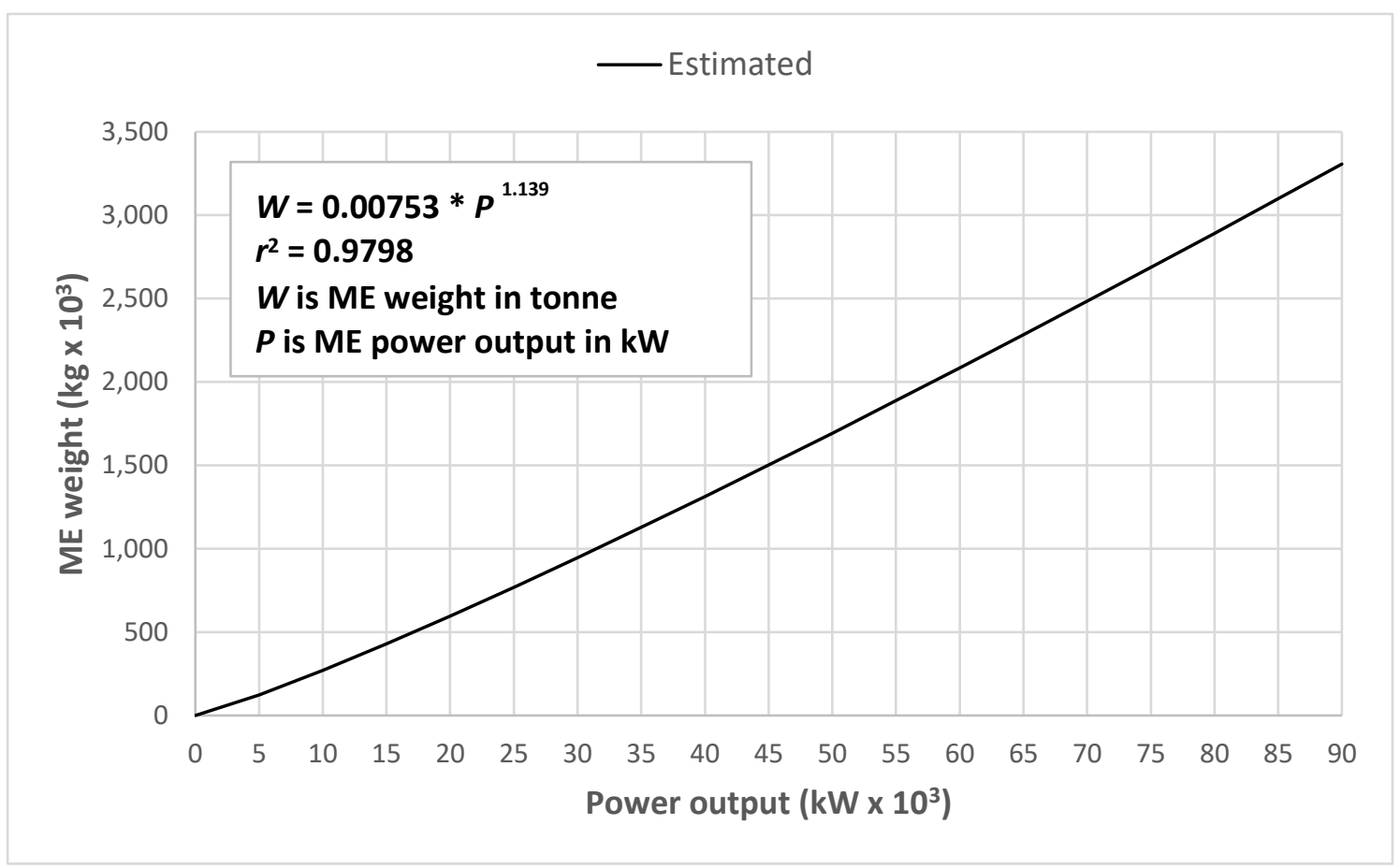

Fig.66 Estimated ME weight versus power output

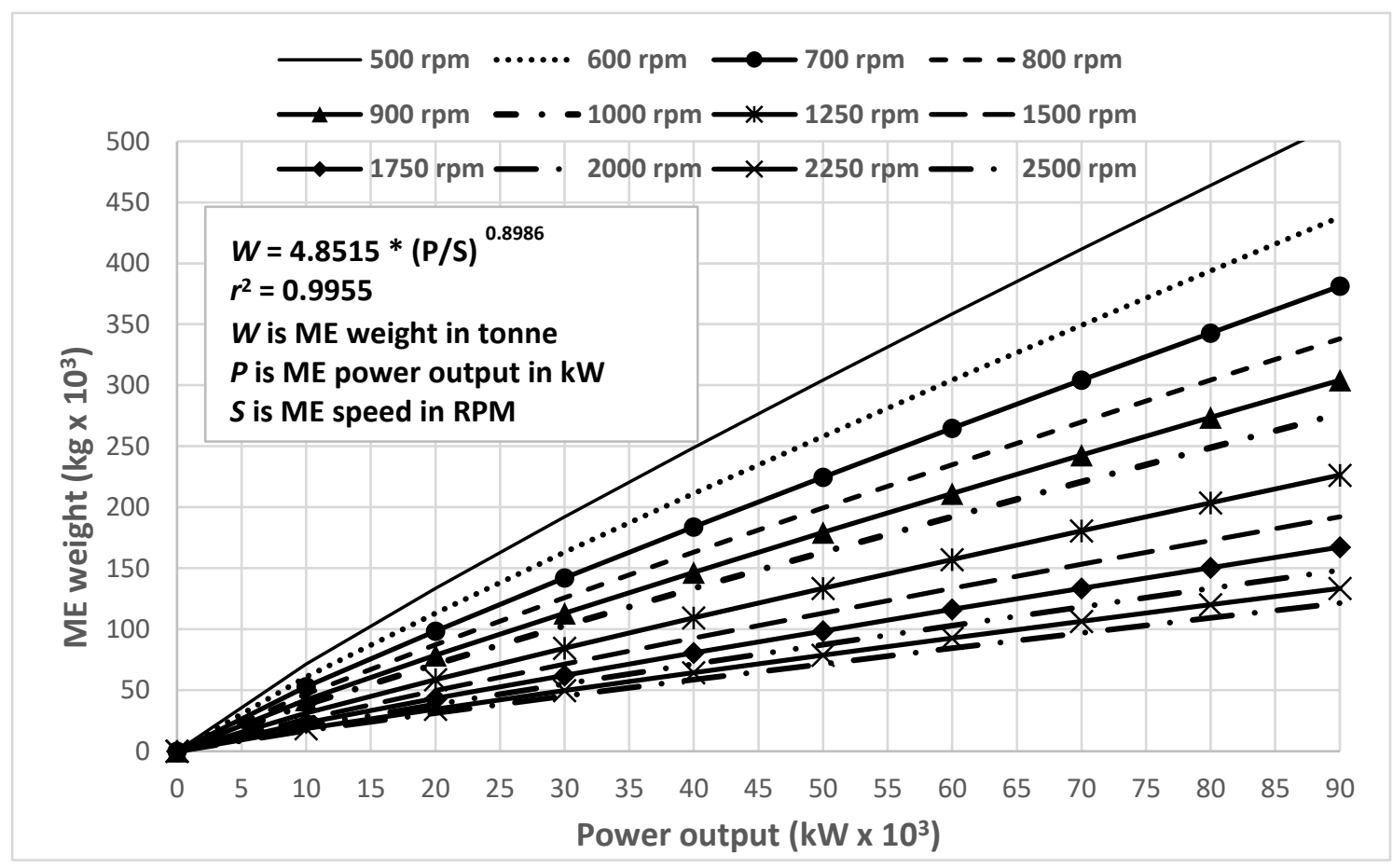

Fig.67 Estimated ME weight versus power output at a constant speed

One may choose to use a similar approach to estimate M.E. weight under speed groups. Accordingly, Figs. 68 and 69 are developed using Figs. 25 and 31, respectively, for the low-speed group. Figures 70 and 71 are developed using Figs.35 and 41 respectively for medium speed group. Figures 72 and 73 are developed using Figs.45 and 51, respectively, for high- speed group. 


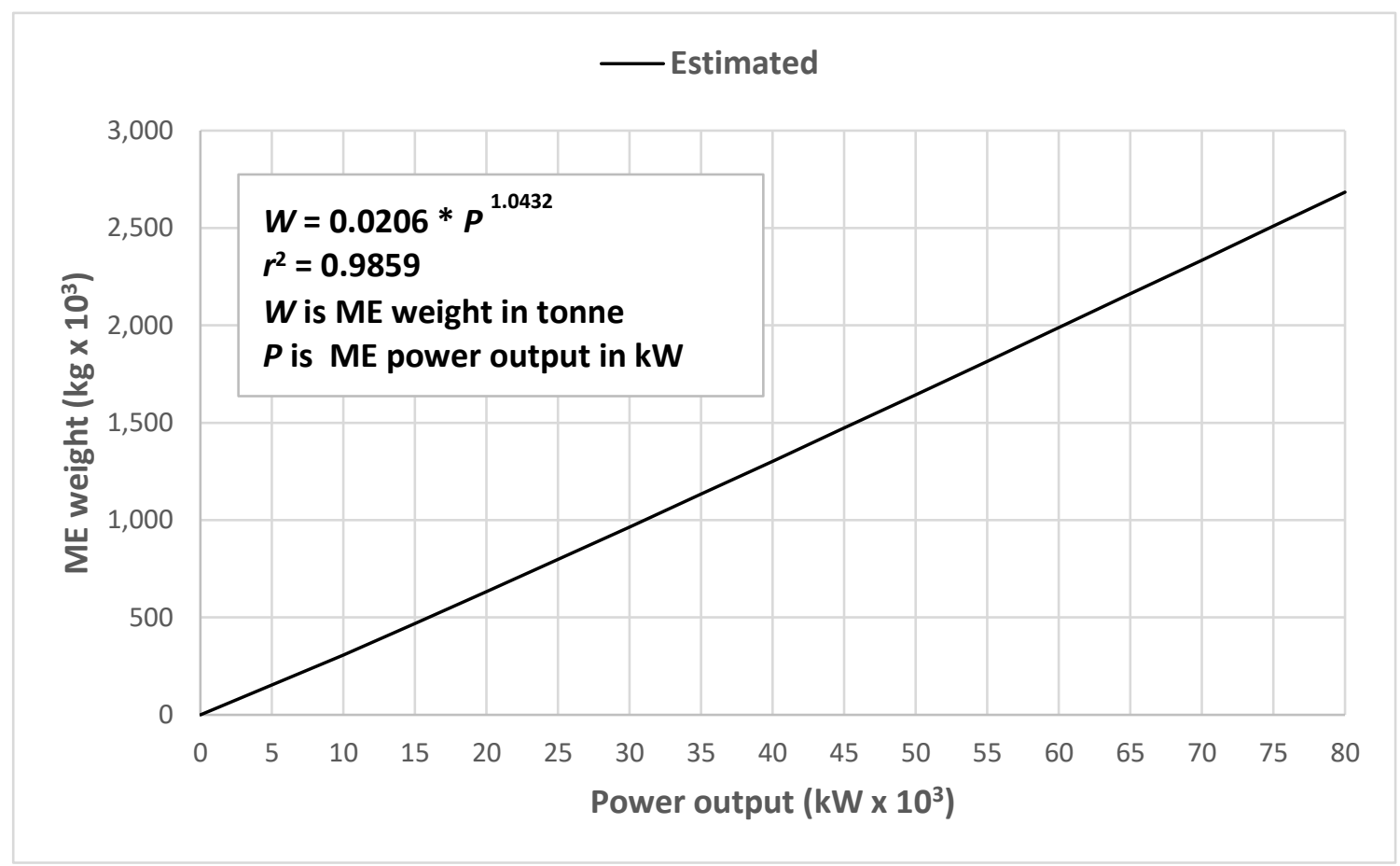

Fig.68 Estimated ME weight versus power output under the low-speed group

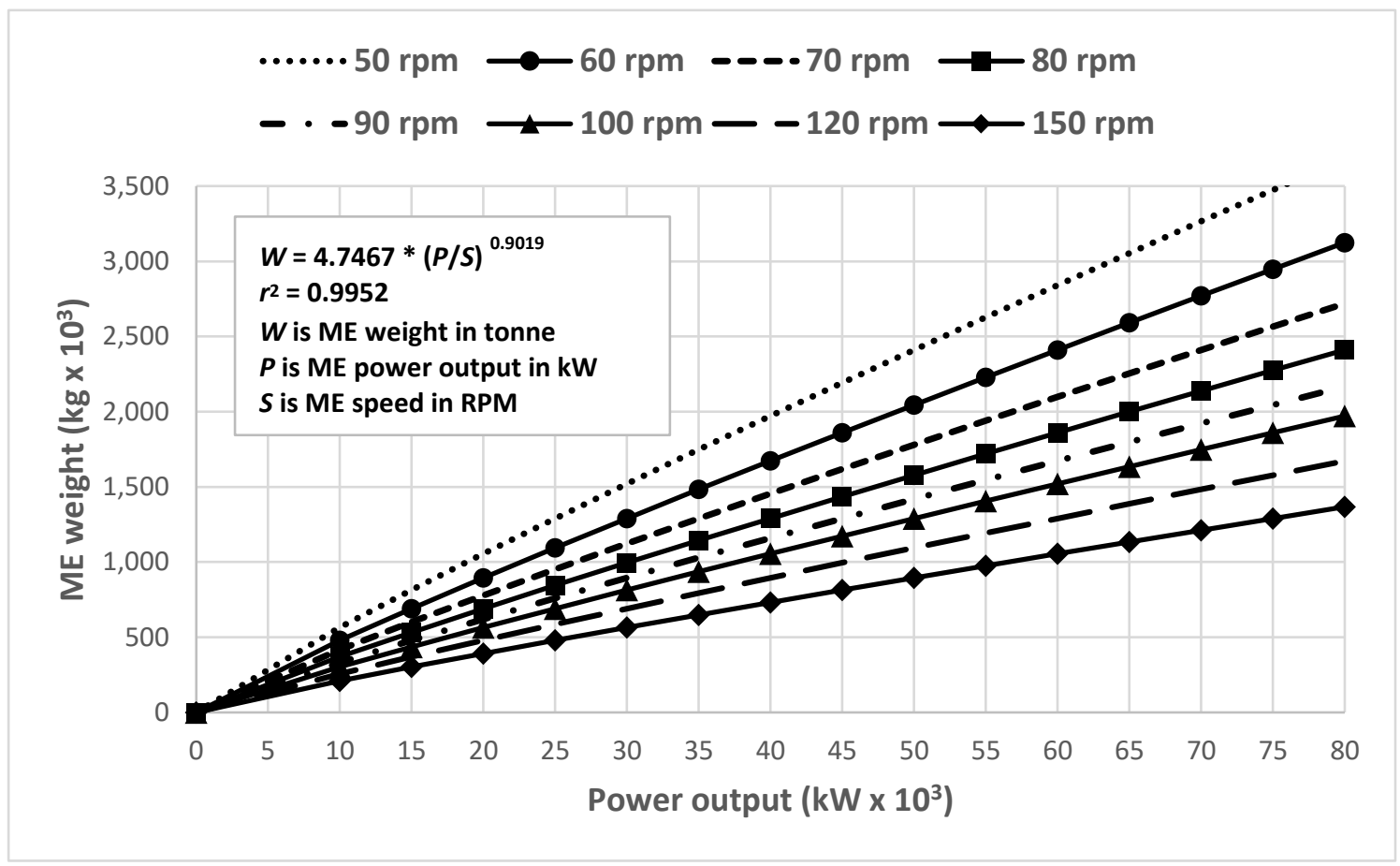

Fig.69 Estimated ME weight versus power output at a constant speed under the low-speed group 


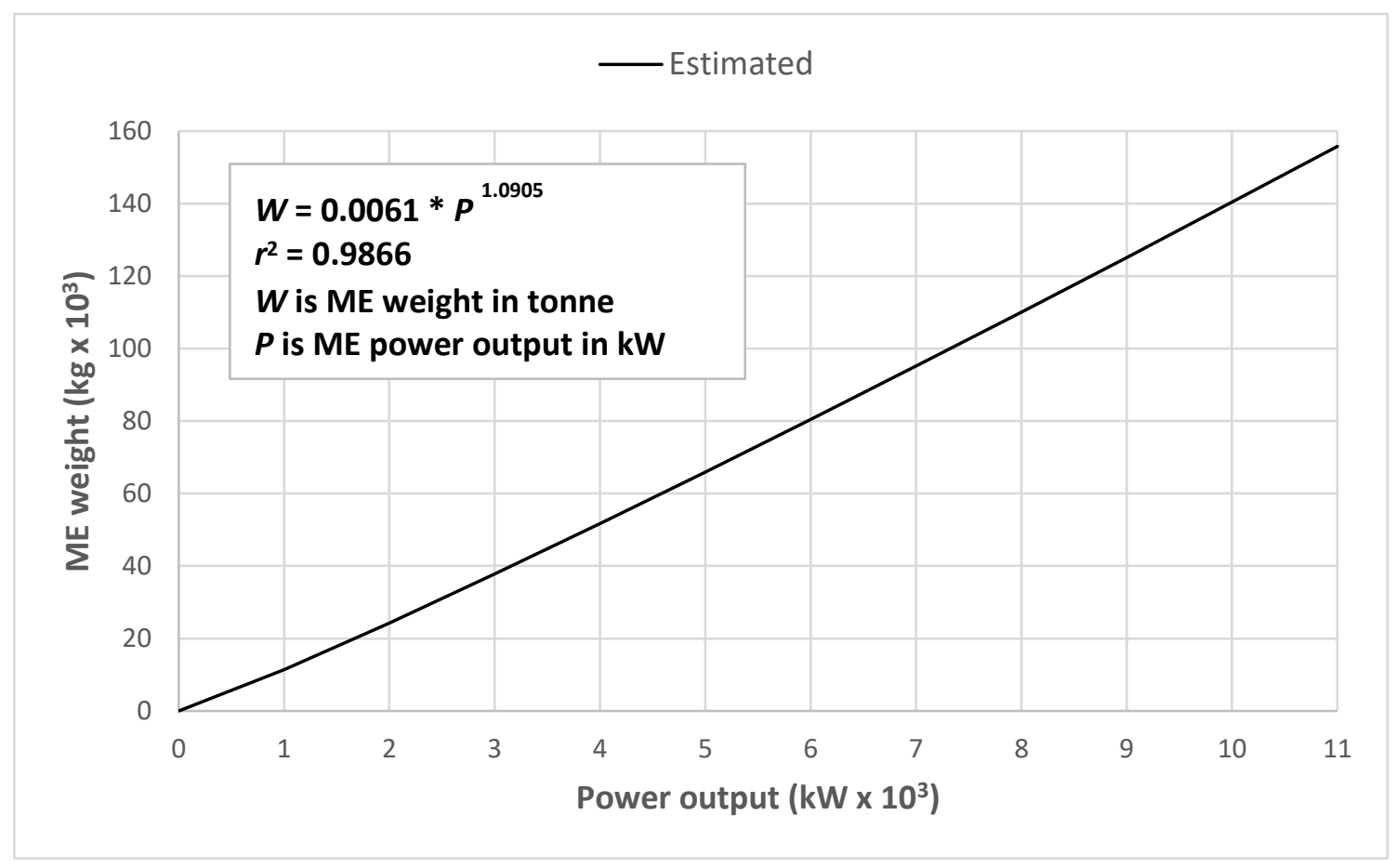

Fig.70 Estimated ME weight versus power output under the medium-speed group

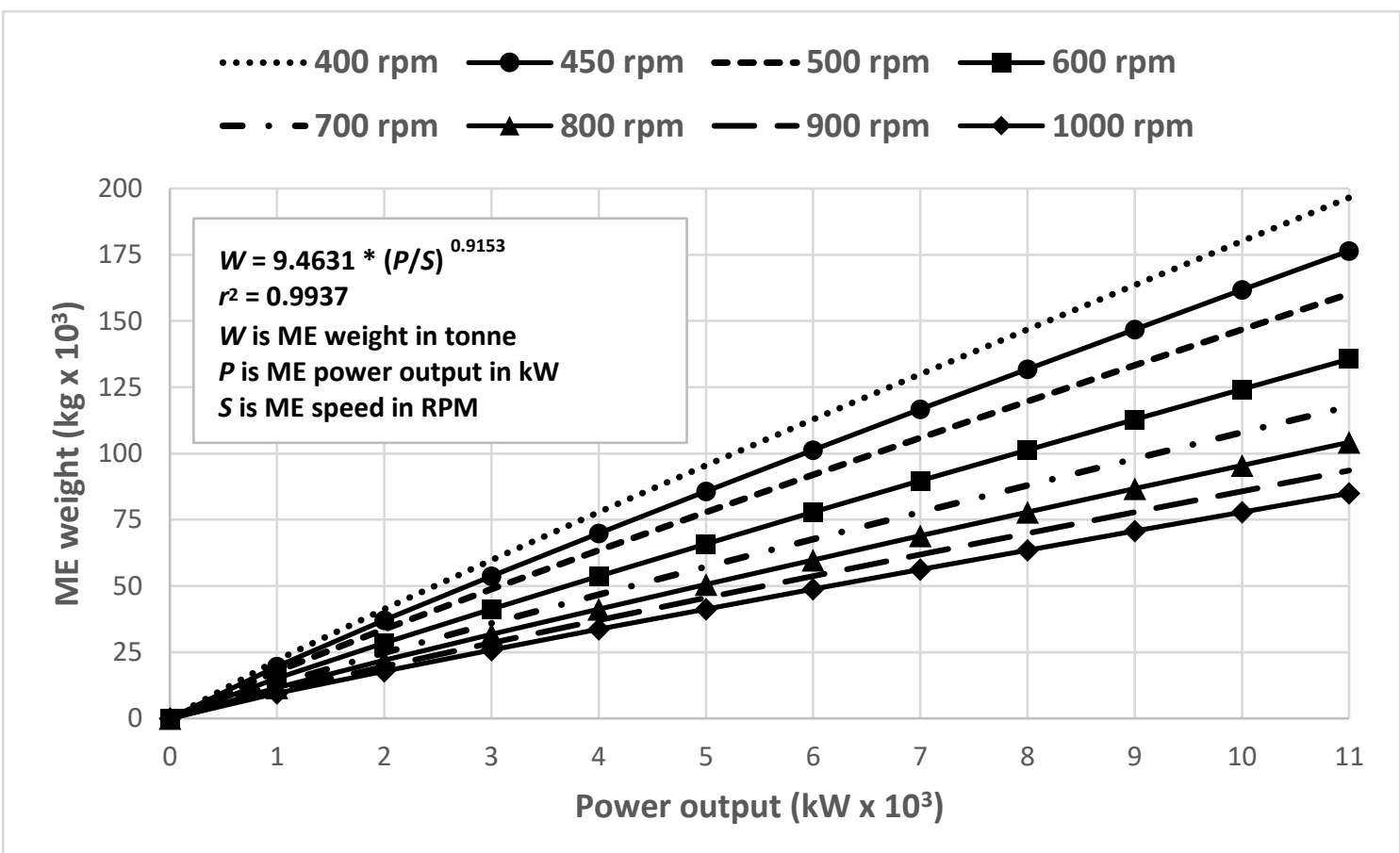

Fig.71 Estimated ME weight versus power output at a constant speed under the medium-speed group 


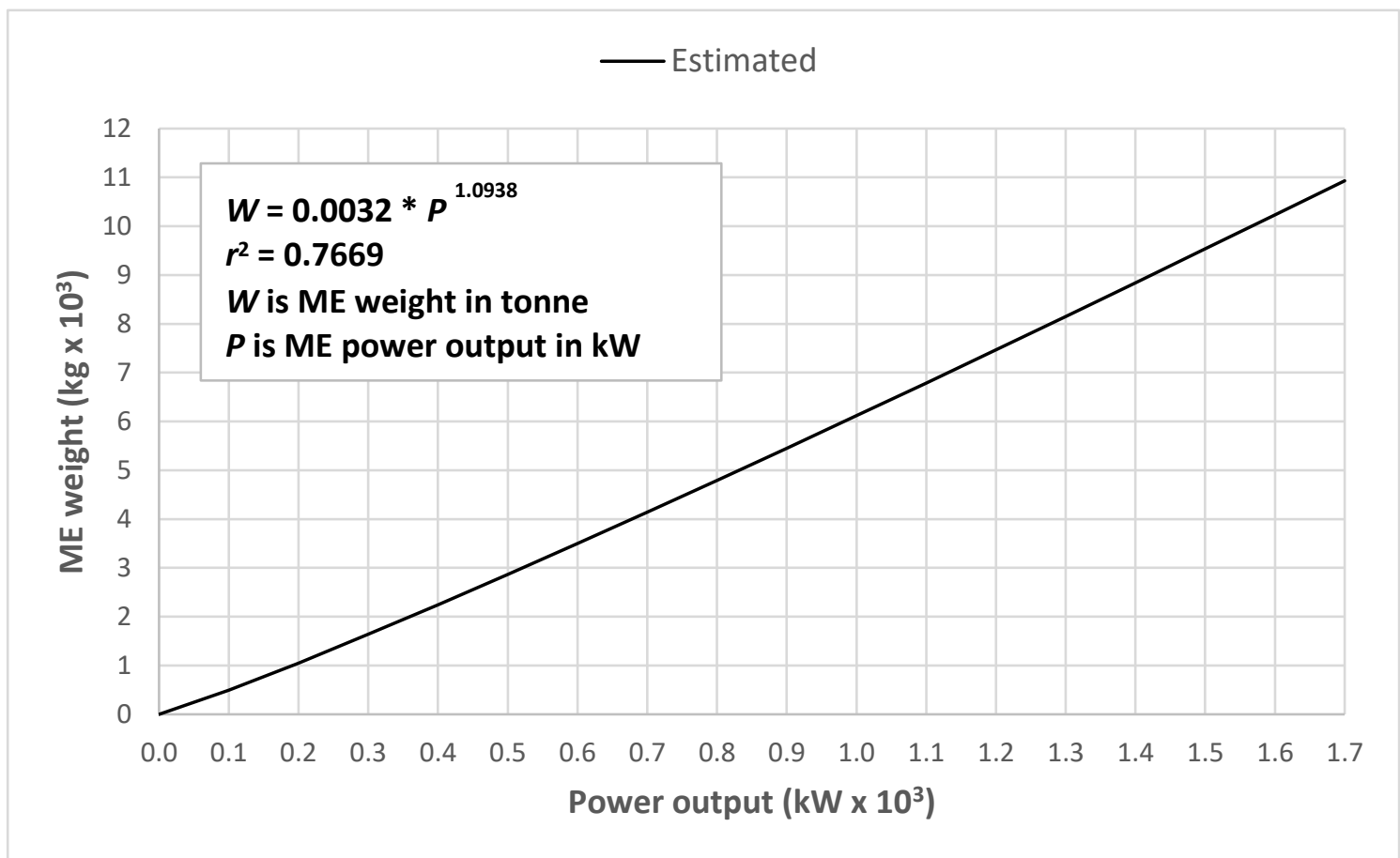

Fig.72 Estimated ME weight versus power output under the high-speed group

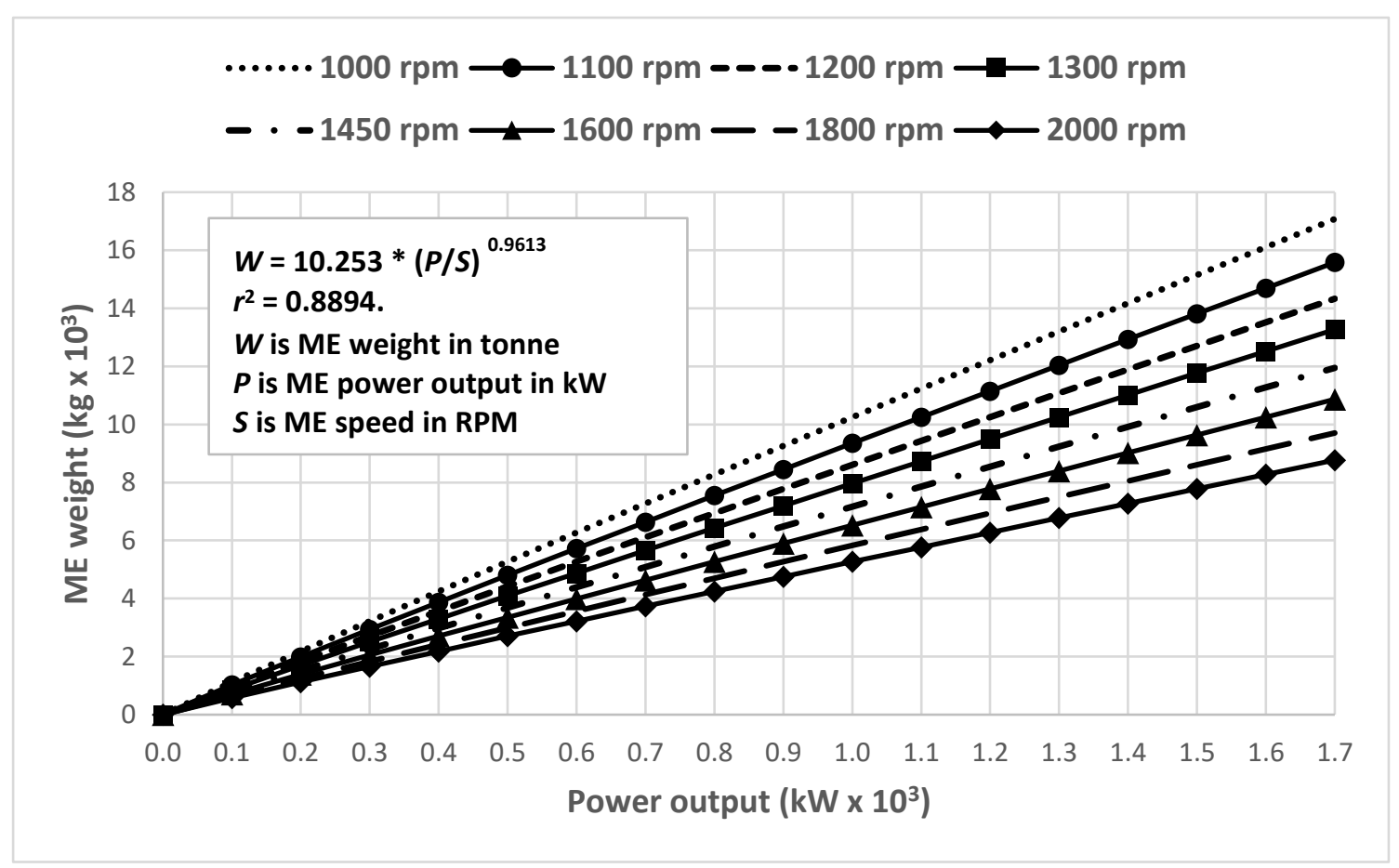

Fig.73 Estimated ME weight versus power output at a constant speed under the high-speed group

\subsubsection{Auxiliary Engine}

Table 20 suggests that the weight-power relationship (Fig.55), weight-power-RPM ratio relationship (Fig.61) and weight-power-RPM ratio per cylinder relationship (Fig.63) yield the highest correlation coefficients under power equation form. It is evident from the validation results (Table 29) that the proposed mathematical model (equation 14) yield a wide range of errors (\%) and high standard 
deviation (\%) which will affect the accuracy of the estimation of engine weight. The validation results of various relationships under different equations (Table 31) dictate that the power equation yields the lower range of error (\%) and a smaller standard deviation (\%). Table 33 displays a summary of the above findings. It is very clear from the Table that the above mentioned three relationships under power equation form yield the optimum result in terms of mentioned statistical parameters. During the preliminary and conceptual design stage, the designers have only two options to estimate the engine weight, i) based on generator power $(\mathrm{kW})$, ii) based on generator power $(\mathrm{kW})$ and engine speed (RPM). The cylinder number is not useful at that stage. As such, weight-power (Fig.55) and weightpower-RPM ratio (Fig.61) relationships are the most appropriate to estimate the engine weight.

Table 33 Summary of the validation result

\begin{tabular}{|r|r|r|r|}
\cline { 2 - 4 } \multicolumn{1}{c|}{} & $W=\mathrm{a} * P^{\mathrm{n}}$ & $W=\mathrm{a} * T^{\mathrm{n}}$ & $W=\mathrm{a} *(T / N)^{\mathrm{n}}$ \\
\hline Item & Fig.55 & Fig.61 & \multicolumn{1}{c|}{ Fig.63 } \\
\hline Maximum error (\%) & 252 & 174 & 210 \\
\hline Minimum error (\%) & -48 & -49 & -55 \\
\hline Range (\%) & 300 & 223 & 265 \\
\hline Mean (\%) & 23 & 5 & 16 \\
\hline Variance (\%) & 3,112 & 1,201 & 1,881 \\
\hline Standard deviation (\%) & 56 & 35 & 43 \\
\hline
\end{tabular}

Accordingly, Fig.74 is developed using the relationship of weight-power output of auxiliary engines under power equations (Fig.55). It shows the relationships between the auxiliary engine's estimated weight and the generator's calculated power output under the power equation.

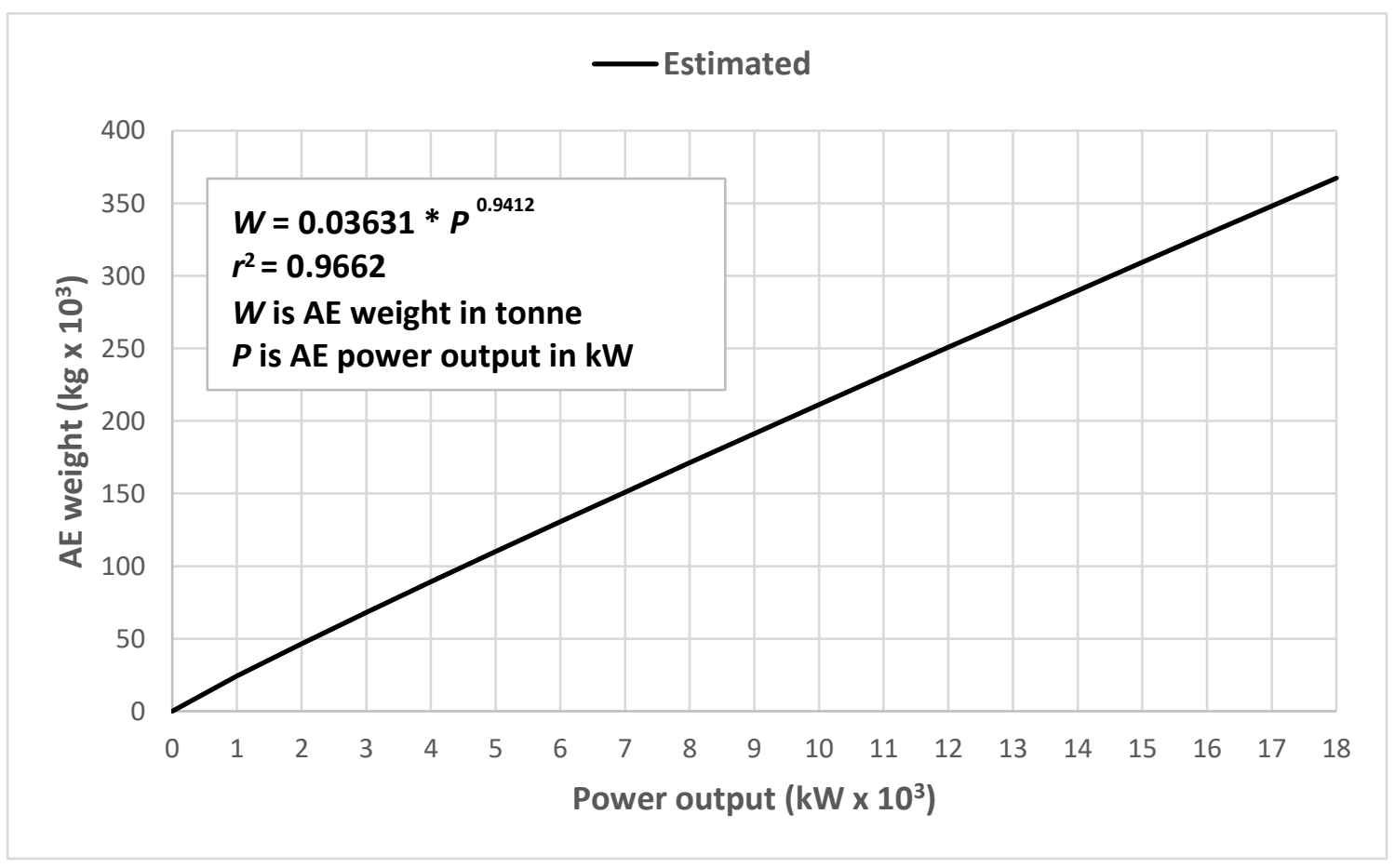

Fig.74 Estimated AE weight versus power output 
Figure 75 is developed using the relationship of weight and a power-RPM ratio of auxiliary engines under power equations (Fig.61) for various constant RPMs. It is clear from the Fig.75 that for a particular generator output, the auxiliary engine's weight decreases with the increase of engine RPM. This is in line with the initial assumption.

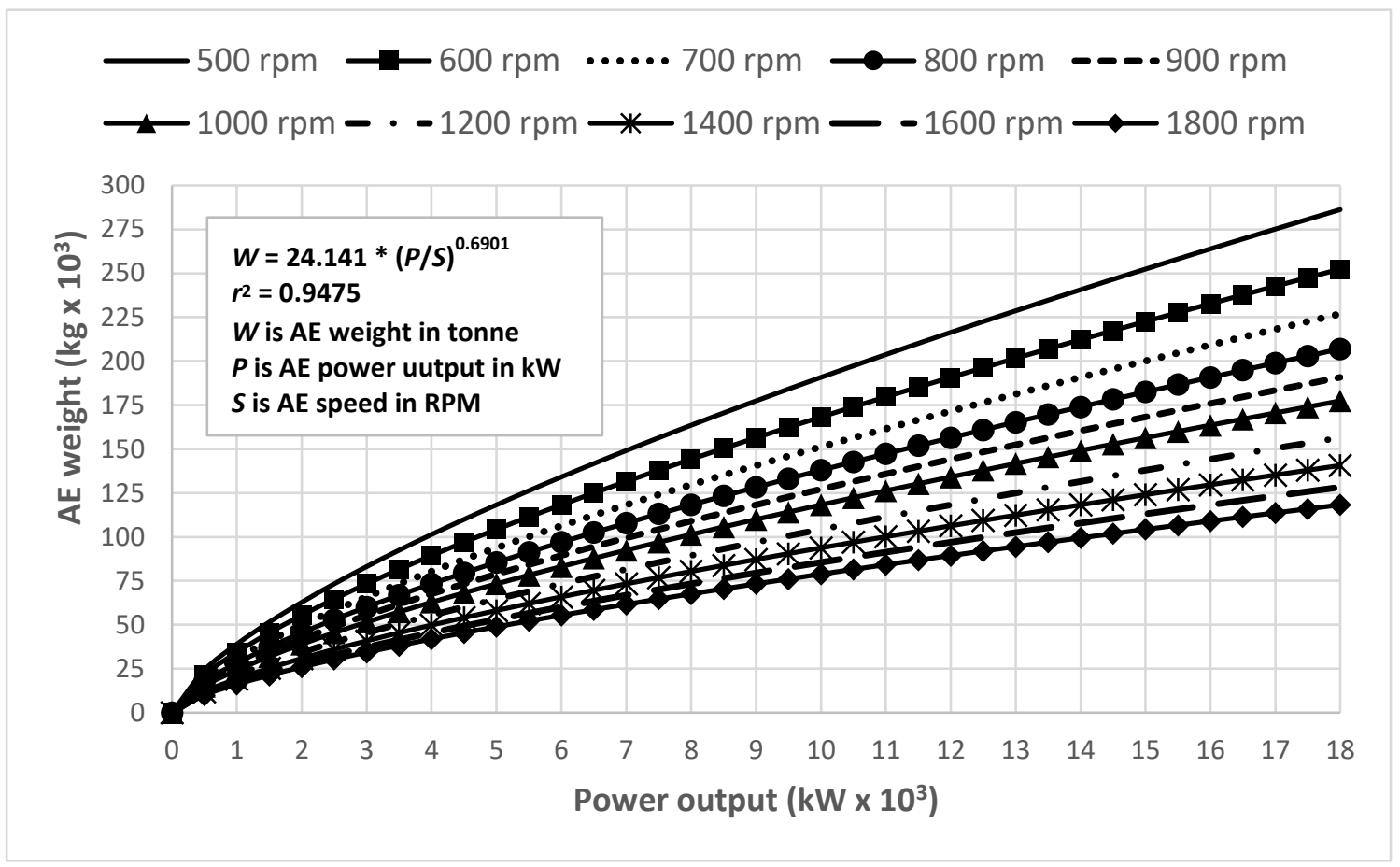

Fig.75 Estimated AE weight versus power output at a constant speed

\section{Marine propulsion machinery weight}

For estimating the propulsion engine weight, the following options may be followed.

\section{Option - I}

Use the power output at $100 \%$ MCR (calculated value during the conceptual design stage) to estimate the engine weight (Fig.66) irrespective of speed

\section{Option - II}

Use the power output at 100\% MCR (calculated value during the conceptual design stage) and designated engine RPM (designers may have some reservations about RPM) to estimate the main engine weight (Fig.67).

\section{Option III}

Use the power output at 100\% MCR (calculated value during the conceptual design stage) and designated engine RPM (designers may have some reservations about RPM) to estimate the main engine weight under speed groups as appropriate (Fig.68-73).

\section{Option IV}

Use the regression equations $10,11,12,13$ as appropriate. 


\section{Power generation machinery weight}

For estimating the diesel generator weight, the following options may be followed.

Option - I

Use the generator's power output at $100 \%$ MCR (calculated value during the conceptual design stage) to estimate the auxiliary engine weight (Fig.74) irrespective of speed.

Option - II

Use the power output of the generator at $100 \%$ MCR (calculated value during the conceptual design stage) and designated engine RPM (designers may have some reservations about RPM) to estimate the auxiliary engine weight (Fig.75).

\section{Option III}

Use the regression equation 14

\subsection{Conclusion}

This article investigates the trends of the main propulsion engines' weight and auxiliary engines regarding power output, cylinder number, RPM, power-RPM ratio, and power-RPM ratio per cylinder. Based on the analyses' results, the engine's weight (main engine and auxiliary engine) significantly depends positively on power output, cylinder number, power-RPM ratio, and power-RPM ratio per cylinder and negatively on RPM but at different degrees of responses. All independent variables are linearly (linear equation form), exponentially (power equation form) and polynomial form (secondorder) associated with the dependent variable. Hence, it can be concluded with sufficient confidence level, that power output, cylinder number, RPM, power-RPM ratio, and power-RPM ratio per cylinder jointly or independently have a significant impact on the weight but at different degrees of response. It is important to note that only the main engine and generator's required power output is available during the conceptual and preliminary design stage. In some cases, the owner may choose the RPM range of the main engines. Figures 66-73 and 74-75 may help estimate the main engine and auxiliary engine weights.

Despite the limitations of sample size, this research's various outcomes may be useful to ship designers as a guiding tool. Using these findings, ship designers may estimate the weights of a propulsion engine and an auxiliary engine for initial stability calculations during the conceptual and preliminary design of a ship. These works can be fine-tuned further with a larger sample size. For future study, artificial intelligence, neural networking may be considered to develop and validate the model.

\section{Acknowledgement}

Product specifications of main engines and auxiliary engines of various manufacturers, available online and hardcopy through personal contact, are broadly acknowledged. 


\section{References}

Dev, A.K. and Saha, M. 2015 Modeling and Analysis of Ship Repairing Time, Journal of Ship Production and Design, Vol.31, No.2, pp. 129-136.

Dev, A.K. and Saha, M. 2016 Modeling and Analysis of Ship Repairing Labor, Journal of Ship Production and Design, Vol.32, No.4, pp. 258-271.

D.G.M. Watson 1998 Practical Ship Design, ELSEVIER

Murray, R.S. 1992 Theory and Problems on Statistics, McGraw-Hill International, UK.

Papanikolaou, A. 2014 Ship Design Methodologies of Preliminary Design, Springer, pp. 180-181.

Roh, M.I. and Lee, K. Y. 2018 Computational Ship Design, Springer, pp.

Schneekluth, H. and Bertram, V. 1998 Ship Design for Efficiency and Economy, Elsevier, pp.

Walpole, R. E. and Myers, R. H. 1978 Probability and Statistics for Engineers and Scientists, Macmillan Publishing Co., Inc., New York.

Watson and Gilfillan, 1976, Some Ship Design Methods, R.I.N.A.

Watson, 1962, Estimating Preliminary dimensions in ship Design, Trans. IESS, Vol.105, 1962 